\title{
Characterization and Application of Self-Assembled Nano- Materials for Fluid Steering and Separation in Microfluidics
}

Xingwei Wu

West Virginia University

Follow this and additional works at: https://researchrepository.wvu.edu/etd

\section{Recommended Citation}

Wu, Xingwei, "Characterization and Application of Self-Assembled Nano-Materials for Fluid Steering and Separation in Microfluidics" (2013). Graduate Theses, Dissertations, and Problem Reports. 5013.

https://researchrepository.wvu.edu/etd/5013

This Dissertation is protected by copyright and/or related rights. It has been brought to you by the The Research Repository @ WVU with permission from the rights-holder(s). You are free to use this Dissertation in any way that is permitted by the copyright and related rights legislation that applies to your use. For other uses you must obtain permission from the rights-holder(s) directly, unless additional rights are indicated by a Creative Commons license in the record and/ or on the work itself. This Dissertation has been accepted for inclusion in WVU Graduate Theses, Dissertations, and Problem Reports collection by an authorized administrator of The Research Repository @ WVU.

For more information, please contact researchrepository@mail.wvu.edu. 
Characterization and Application of Self-Assembled Nano-Materials for Fluid Steering and Separation in Microfluidics

\author{
by \\ Xingwei Wu \\ Dissertation submitted to the Eberly College of Arts and Sciences \\ at West Virginia University \\ in partial fulfillment of the requirements \\ for the degree of \\ Doctor of Philosophy \\ in \\ Chemistry
}

Approved by

Dr. Lisa Holland, Committee Chairperson

Dr. Parviz Famouri

Dr. Fred King

Dr. Ronald Smart

Dr. Michelle Richards-Babb

Chemistry Department

Morgantown, West Virginia

2013

Keywords: Phospholipids, Viscosity, Microfluidics, Valve, Non-Newtonian fluid, Hydrodynamic injection

Copyright $@ 2013$ Xingwei Wu 


\begin{abstract}
Characterization and Application of Self-Assembled Nano-Materials for Fluid Steering and Separation in Microfluidics
\end{abstract}

\title{
Xingwei Wu
}

Aqueous phospholipid preparations comprised of 1,2-dimyristoyl-sn-glycero-3phosphocholine (DMPC) and 1,2-dihexanoyl-sn-glycero-3-phosphocholine (DHPC) are prevalent materials for biological characterization. The viscosity behavior of DMPC/DHPC was characterized as a function of $\mathrm{q}$ value ( $\mathrm{q}=[\mathrm{DMPC}] /[\mathrm{DHPC}]$ ), hydration as well as temperature firstly on bench-top and then in microfluidic chip. The rheology of $20 \%$ phospholipid preparations of [DMPC]/[DHPC] $=2.5$ reveals that, under conditions utilized for fluid steering, the materials are shear-thinning power-law fluids with a power-law index ranging from 0.30 through 0.90 . Phospholipid preparations are utilized to steer fluids in microfluidic chips and support hydrodynamic delivery of sample across a double T injection region in a chip. The fact that the phospholipids are fully integrated as a valving material as well as a separation medium is demonstrated through the separation of linear oligosaccharides labeled with 1-aminopyrene-3,6,8-trisulfonic acid. 


\section{DEDICATION}

To my beloved husband, Bruce Tepke and my parents, Shutian Wu and Yayan Li, I dedicate this dissertation with great love and appreciation. 


\section{ACKNOWLEDGMENTS}

First and foremost, I would like to express my deepest gratitude to my advisor, Dr. Lisa Holland, for her caring, mentoring and motivating throughout the years. Besides the knowledge and research method I've learned from her, the strategy to get things done as well as the fearless spirit is truly inspiring and life applicable.

I would like to thank Dr. King, Dr. Smart, Dr. Famouri, Dr. Carroll and Dr. RichardsBabb for serving as my committee members and guiding my research for the past several years. Special thanks go to Dr. Michelle Richards-Babb, who agreed to be on my committee at last moment and provided me with opportunities to develop my teaching skills.

I would like to thank my previous labmates Dr. Theron Pappas, Dr. Liliya Bykova, Dr. Christian White, Dr. Ruijuan Luo, Dr. Ted Langan and Dr. Stephanies Archer-Hartman and current labmates: Brandon Durney, Vincent Nyakubaya, Tyler Davis, Anthony Moncrief and Dr. Sri Yedlapalli for all the help and assistance and all the time we spent together.

I would also like to thank professors in the chemistry department whom I took class from for helping me to develop my academic background; the staff working in stockroom and main office in the department for all the assistance they provided. I especially would like to thank Allen Burns, Randall Eaglen and Sherman Adams for their help with custombuilt instruments. My research would not have been possible without their helps.

I gratefully acknowledge National Science Foundation Grant No. CHE0749764 and the graduate program of Eberly College of Arts and Science for funding me to attend and present my work at annual academic conferences. 
Chapter 1

Introduction$-1$

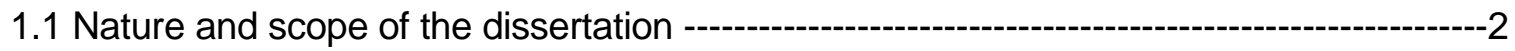

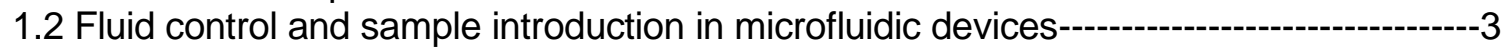

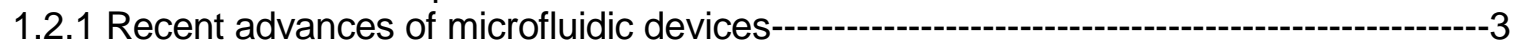

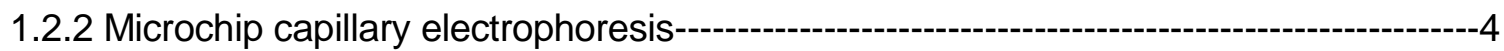

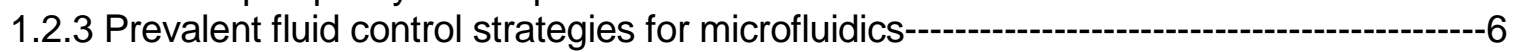

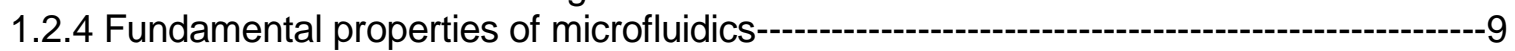

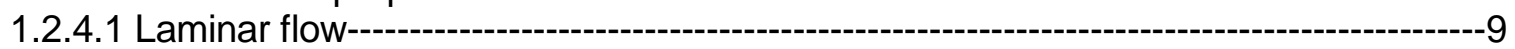

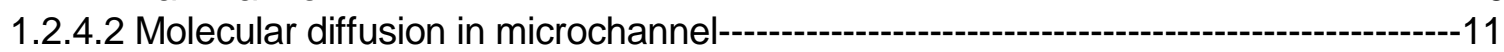

1.2.4.3 Enhanced diffusion of pressure driven flow in microchannel----------------------------12

1.2.5 Practical difficulties of fluid control via direct pressure push--------------------------------15

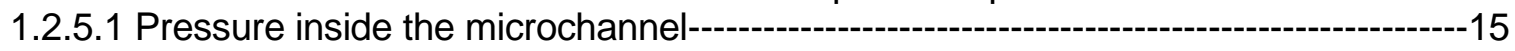

1.2.5.2 Volume mismatch of the microchannel and the real world connection----------------16

1.3 Phospholipids: a novel responsive material for non-mechanical fluid control

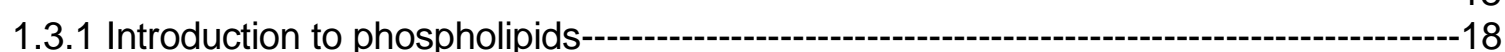

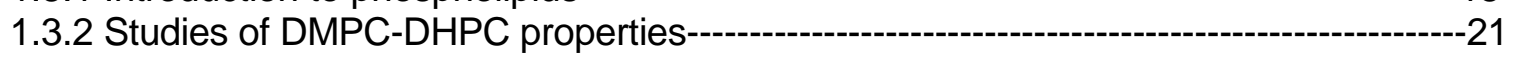

1.3.2.1 Microscale approaches: morphological study of DMPC-DHPC----------------------23

1.3.2.2 Macroscale approach: viscosity behavior of DMPC-DHPC-----------------------------25

1.4 Multi-purpose use of phospholipids in microfluidic systems----------------------------------28

References ------------------------------------------------------------------------------------------------29

Chapter 2

Viscosity Characterization of DMPC-DHPC Phospholipid Preparations in

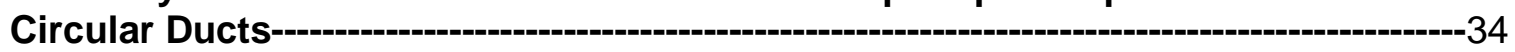

2.1 Introduction: viscosity of phospholipid preparations-------------------------------------35

2.1.1 The importance of viscosity-------------------------------------------------------36

2.1.2 Definition of viscosity and non-Newtonian fluid-------------------------------------------37

2.1.3 Viscosity characterization of non-Newtonian fluid--------------------------------------38

2.1.3.1 Capillary viscometer------------------------------------------------------------------38

2.1.3.2 Measurement of apparent viscosity of phospholipids with

capillary viscometer----------------------------------------------------------------------------------39

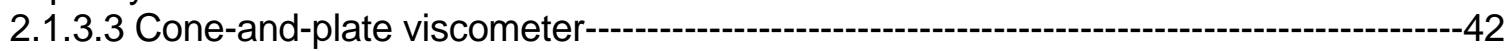

2.1.4 Custom built system for viscosity measurement of phospholipids-----------------------45

2.1.5 Temperature control with thermoelectric module (TEM)----------------------------50

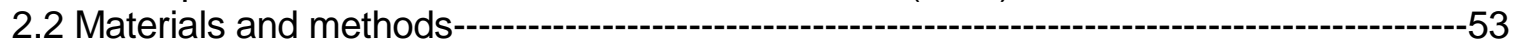

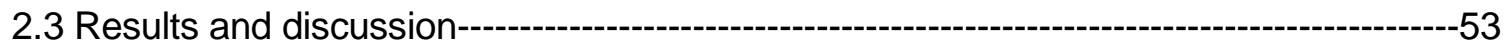

2.3.1 Result of viscosity measurement in circular ducts------------------------------------53

2.3.2 Problems with bench-top measurement result------------------------------------------------60

2.3.3 Sources of error due to the flaws in the experimental setup-----------------------61

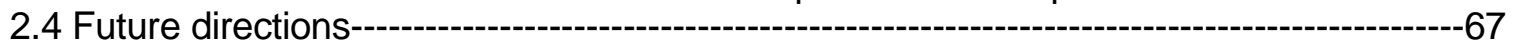




\section{Chapter 3}

Viscosity Measurements of DMPC-DHPC Phospholipid Preparations in Trapezoidal Microfluidic Channel -------------------------------------------------70

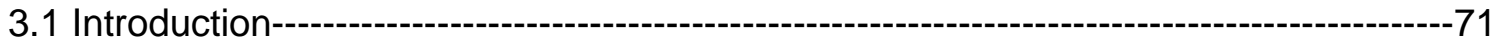

3.2 Materials and methods-----------------------------------------------------------------------72

3.2.1 Reagents and materials----------------------------------------------------------------------72

3.2.2 Glass chip fabrication--------------------------------------------------------------------------73

3.2.3 Measurement of apparent viscosity in non-circular ducts------------------------------75

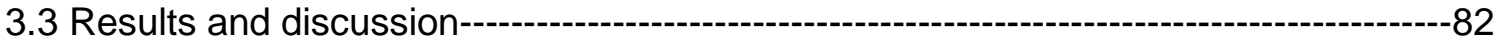

3.3.1 An example of data processing--------------------------------------------82

3.3.2 Non-Newtonian flow in trapezoidal cross section channel-----------------------------87

3.3.3 Results of viscosity measurement of phospholipids in microfluidic channel

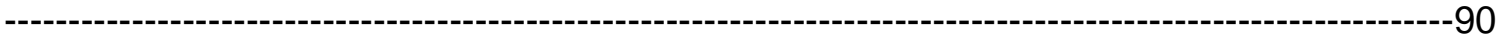

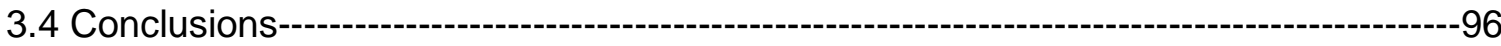

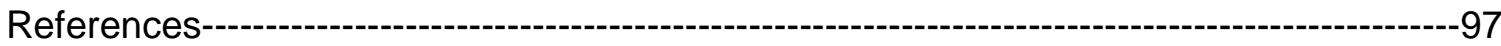

\section{Chapter 4}

The Use of Phospholipids for Non-Mechanical Flow Control and Injection in Microfluidics----

4.1 Introduction----------------------------------------------------------------------------------------99

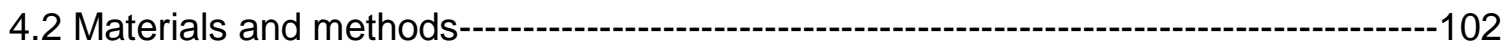

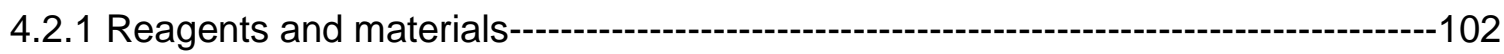

4.2.2 Beckman P/ACE Capillary Electrophoresis instrument----------------------------------109

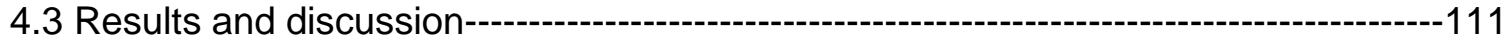

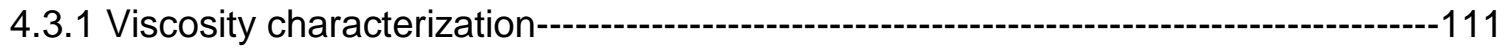

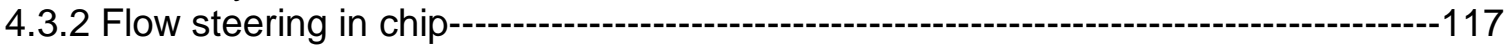

4.3.3 Thermally facilitated hydrodynamic injections--------------------------------------------121

4.3.4 Hydrodynamic sample introduction and capillary electrophoresis separation

-----------------------------------------------------------------------------------------------------------------123

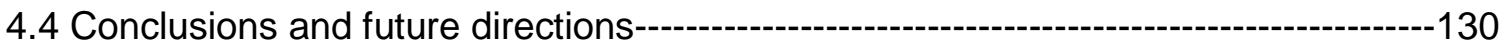

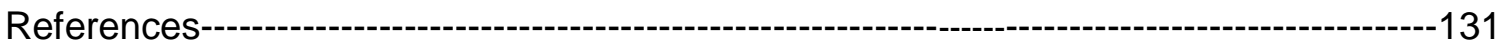

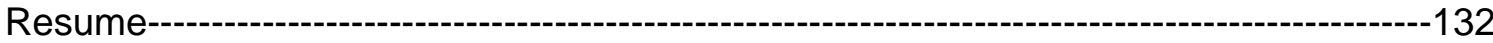




\section{LIST OF FIGURES}

Figure 1-1 Schematics showing the mechanism of the first generation, push down design pneumatic PDMS valve

7

Figure 1-2 Two-dimensional drawings demonstrating the parabolic flow pattern of laminar flow and turbulent flow in the microfluidic channel

Figure 1-3 A Band broadening resulting from the laminar flow profile

and longitudinal diffusion....

Figure 1-3B A hyperthetical example of the case in Figure 1-3 A

If it were possible to obtain data in the absence of laminar flow.

Figure 1-4 Capture fluorescenc microscope image showing the

Taylor dispersion of the sample plug

Figure 1-5 Structure of DMPC

Figure 1-6 Structure of DHPC 20

Figure 1-7 Various models of DMPC/DHPC assembly 22

Figure 2-1 A schematic of pressure driven flow in a circular tube 41

Figure 2-2 A schematic of a cone-and-plate viscometer. 44

Figure 2-3 A photograph showing the components of the custom-built pressure system......

Figure 2-4 A picture of the custom made pressure system . 48

Figure 2-5 A schematic of the custom-built pressure reservoir 49

Figure 2-6 Diagram of a TEM 51

Figure 2-7 A photograph showing the other components of a TEM temperature control system.

Figure 2-8 Plots of apparent viscosity vs. shear rate for q 2.5, 20\% phospholipid in circular ducts

Figure 2-9 Illustration of how errors result from the fact that one tenth of the capillary was not temperature controlled......

Figure 3-1 Cross sectional view showing the glass chip fabrication process.

Figure 3-2 Instrument setup for measurement of viscosity in chip

Figure 3-3 Schematics of the setup and temperature control for viscosity measurement in chip 78 
Figure 3-4 Top view of the chip design used for on-chip viscosity measurement

Figure 3-5 A photograph showing the setup for viscosity measurement in microfluidic chip

Figure 3-6A Photograph showing the temperature regulation of the pressure reservoir and the PEEK tubing connecting the pressure reservoir to the glass chip

Figure 3-7 A plot of apparent viscosity of a $20 \% \mathrm{q}=2.5$ preparation with change in shear rate in microfluidic chip. 93

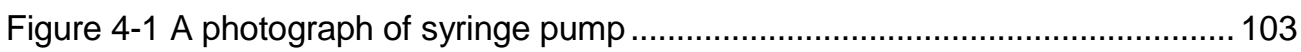

Figure 4-2 LabSmith syringe pump............................................................ 104

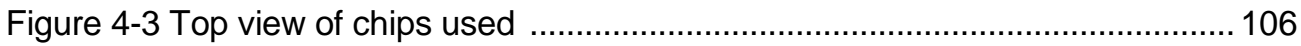

Figure 4-4 A photograph showing a microfluidic chip on the microscope stage

Figure 4-5 A plot displaying the apparent viscosity of a 20\% [DMPC]/[DHPC] $=2.5$

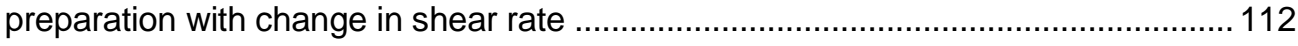

Figure 4-6 Steps and captured images of repetitive flow switching........................ 117

Figure 4-7 Steps and captured images for sequential flow switching .................... 119

Figure 4-8 Images captured during one cycle of 10 manual repetitive

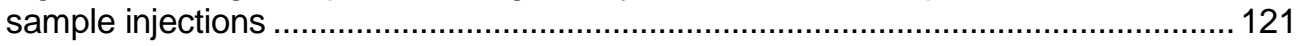

Figure 4-9 Sample introduction with thermal control.......................................... 123

Figure 4-10 The capillary electrophoresis separation obtained with the method

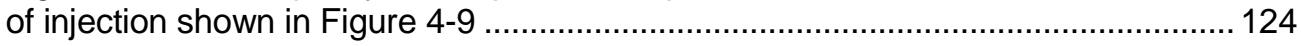

Figure 4-11 A negative control to demonstrate the difficulty of hydrodynamic injection in microfluidics. 125

viii 


\section{LIST OF TABLES}

Table 2-1 Power law fit for circular ducts of different diameter.

.56

Table 2-2 Apparent viscosity calculated for $20 \%$ phospholipid in circular ducts.....

Table 2-3 Apparent viscosity for $15 \%$ and $20 \%$ phospholipid in 50

$\mu \mathrm{m}$ circular ducts.. 58

Table 3-1A-E Step-by-step calculation results of an example of data processing...... 82

Table 3-2 Fitting results of $\mathrm{K}$ using different methods..... 88

Table 3-3 Apparent viscosity calculated for each shear rate for trapezoidal ducts (q 2.5, 20\%)

Table 3-4 Comparison of $K$ and $n$ value from viscosity measurement of DMPC-DHPC preparations (q 2.5, 20\%) between bench-top instrument and microfluidic chip (before turn and after turn) at three temperature

Table 4-1 Apparent viscosity for $20 \%$ phospholipids in microfluidic ducts (PEEK not controlled)

Table 4-2 Comparison of $n$ and $\mathrm{K}$ obtained in trapezoidal ducts ( $q$ 2,5, 20\%) PEEK tubing temperature controlled vs uncontrolled

Table 4-3 Comparison of apparent Viscosity obtained in trapezoidal ducts (q 2.5, 20\%) PEEK tubing temperature controlled vs. uncontrolled ......

Table 4-4 Separation figures of merit for $20 \%$ phospholipid with P/ACE MDCQ..... 128 


\section{LIST OF SYMBOLS / NOMENCLATURE}

1. DMPC - 1, 2-dimyristoyl-sn-glycero-3-phosphocholine

2. DHPC - 1, 2-dihexanoyl-sn-glycero-3-phosphocholine

3. Q-quantity injected in hydrodynamic injection

4. $r$ - the radius of the fluid channel

5. $\Delta \mathrm{P}$ - applied pressure

6. tint_injection time

7. n--viscosity of the sample solution

8. L-length of the channel

9. $C_{i}$-the initial concentration of the sample

10. D-diffusion coefficient

11. x-position

12. W-broadened peak width

13. V-velocity

14. Q-volume flow rate in Hagen-Poiseuille equation

15. PDMS-polydimethylsiloxane

16. PEEK- polyetheretherketone

17. q-[DMPC]/[DHPC]

18. NMR- Nuclear Magnetic Resonance

19. $\mu_{\mathrm{eph}}$ - Electrophoretic mobility

20. E-field strength of the electric field

21. q-charge on the particle

22. r-Stoke's radius

23. TEM-thermalelectric module

24. $\mathrm{V}_{\text {ave }}$-mean linear velocity

25. $L$ - total length of the capillary

26. $\eta_{\mathrm{A}}$-apparent viscosity

27. K-flow consistency index

28. $n$-flow behavior index

29. FITC- fluorescein isothiocyanate

30. MOPS-3-(N-morpholino)propanesulfonic acid

31. APTS-1-aminopyrene-3,6,8-trisulfonic acid

32. $\gamma$-shear rate

33. T-shear stress

34. A-area

35. P-perimeter

$36 \mathrm{D}_{\mathrm{h}}$-hydraulic diamete 


\section{CHAPTER 1}

\section{Introduction}




\subsection{Nature and scope of the dissertation}

Lab-on-a-chip or the micro total analysis system ( $\mu$-TAS) has grown rapidly since the birth of the concept in $1990 .{ }^{1}$ Remarkable advances have been made on the theoretical and instrumental aspects of microfluidic devices used in $\mu$-TAS in addition to the numerous practical applications in multiple disciplines. As will be detailed in the following sections, fluid manipulation and in particular, hydrodynamic fluid delivery for microchip electrophoresis are indispensable to developing a high-functioning complex microfluidic device. An ideal fluid control scheme for microfluidic devices would be simple, robust and compatible with the compact size of the microfluidic system.

Phospholipids have been used frequently in chemical separations due to their unique properties. For example, in capillary electrophoresis they serve as a coating for the fused silica capillary to suppress electroosmotic flow ${ }^{2}$ as well as an additive in the background electrolyte to enhance electrophoretic separation. ${ }^{3,4}$ The feasibility of using the phospholipid mixture comprised of 1,2-dimyristoyl-sn-glycero-3-phosphocholine (DMPC) and 1,2-dihexanoyl-sn-glycero-3-phosphocholine (DHPC) as a thermally responsive material for fluid control in microfluidic devices was first demonstrated in $2008 .^{5}$ The simplicity of this method of non-mechanical fluid control is especially attractive. It serves as the foundation of this research project.

The goal of this research was to utilize phospholipids for fluid steering and sample introduction, as well as to enhance microchip electrophoresis separation. In order to take full advantage of phospholipids, the fundamental rheological characterization of phospholipids was first carried out. 


\subsection{Fluid control and sample introduction in microfluidic devices}

\subsubsection{Recent advances of microfluidic devices}

Microfluidic devices commonly refer to the miniaturized chips that enable one or multiple laboratory functions such as fluid mixing, sample separation and detection. These chips are usually made from glass or polymeric materials, such as polydimethylsiloxane (PDMS), with fluidic channels of various patterns designed to meet different analytical needs. The fluid volumes these devices handle are typically on the order of pico liter to micro liter amounts. Microfluidic devices have become increasingly attractive as they provide benefits that include minimal reagent and power consumption, fast total analysis time, as well as great portability. Applications have been successfully made in many fields and include clinical diagnostics, ${ }^{6}$ genetic analyses, ${ }^{7}$ immunoassays, ${ }^{8}$ and environmental analyses. ${ }^{9,10}$ Recently, innovations in the use of microfluidic devices for environmental analyses have been made on sample cleanup, preconcentration and detection as well as microchip integration. ${ }^{10}$ Satisfactory detection of environmental pollutants and toxins in standards or real world samples have been achieved with detection limits on the order of parts per billion. ${ }^{10}$ Separations in just over 2 minutes have been achieved on chip. ${ }^{11}$ Some initial success in the commercialization of microfluidic devices, particularly, point-of-care devices has been realized. A number of companies targeting at different applications (e.g., detection of drug abuse, cardiovascular disease, cancer and HIVIAIDS) are currently at different stages of commercialization. ${ }^{12}$ 
For all integrated microfluidic systems, fluid control is one of the key components to successful device development. Precise fluid control within confined microfluidic channels is required for sample introduction, transport, and pretreatment as well as separation and detection. Prevalent fluid control methods for microfluidic devices are pneumatic, which are control methods based on elastomers. These are reviewed in the section 1.2.3.

\subsubsection{Microchip capillary electrophoresis}

Among the various applications of microfluidic devices, microchip capillary electrophoresis is particularly successful. ${ }^{13}$ With the reduced fluidic channel length, microchip capillary electrophoresis requires lower voltages to achieve an electrical field equivalent to that of bench-top instruments. In addition, microchip capillary electrophoresis is especially suitable for clinical applications where the sample size is limited. Analytes that have been separated on a microfluidic chip range from small ions to macromolecules. ${ }^{14}$ Glass chips rather than polymeric chips are predominantly used for microchip capillary electrophoresis because of the surface requirement of the electrophoretic separation and because of the chemical properties of glass. Fabrication of the glass chips, which is detailed in Chapter Three, involves standard lithography techniques followed by a wet chemical etching process.

In microchip capillary electrophoresis, reproducible sample injection is critical to quantitative analysis of analyte. The two methods of sample introduction for bench-top capillary electrophoresis are electrokinetic and hydrodynamic introduction. In electrokinetic injection, the sample plug is driven into the channel by electrical force. 
Typically electrokinetic injection is made with a double T design chip in microchip capillary electrophoresis. Electrokinetic injection is easy to implement; however, the amount of sample injected is dependent upon the electrophoretic mobility of each constituent. ${ }^{15}$ Therefore, the injection is biased. Higher mobility ions are preferably injected. In those cases where electrokinetic injection is subject to bias, hydrodynamic sample injection, in which the sample is pushed into the channel via pressure, is desirable. However, the hurdle to using hydrodynamic injection is that, under normal laboratory conditions, it is not practical to hydrodynamically deliver a relatively small sample plug into a microfluidic channel as is discussed in the following sections. In hydrodynamic sample introduction, the quantity injected, Q, is described by Eqn.1-1: ${ }^{13}$

$\mathrm{Q}=\pi r^{2}\left[\Delta \operatorname{Pr}^{2} t_{\text {int }} /(8 \eta L)\right]\left[C_{i}\right]$ Eqn. 1-1 where $r$ is the radius of the fluid channel, $\Delta \mathrm{P}$ is the applied pressure, $t_{\text {int }}$ is the injection time, $\eta$ is the viscosity of the sample solution, $L$ is the length of the channel and $C_{i}$ is the initial concentration of the sample.

In microfluidic systems with commonly used buffers and channel length, the product of the viscosity and the length is such a small value that hydrodynamic injection of a small plug will require precise control of low pressure or flow rate. In addition, as discussed in the following section, fundamental properties and other practical issues make direct hydrodynamic injection into microfluidic devices impractical. Efforts on instrumental design to enable hydrodynamic sample injection for microchip capillary electrophoresis have been made. ${ }^{16}$ The aim of this research is to utilize the temperature tunable 
viscosity behavior of DMPC-DHPC preparations to facilitate hydrodynamic injection on chip.

\subsubsection{Prevalent fluid control strategies for microfluidics}

Pneumatic fluid control has been the most popular flow control method for microfluidics in the past decade. ${ }^{17-20}$ In this method of flow control, a displacement chamber overlaps with the fluidic channel and is separated by an elastomeric membrane. The actuation is realized by applying pressure or vacuum to deflect the elastomeric membrane into the displacement chamber. A normally open pneumatic valve using soft lithography was first developed by Quake et al in $2000 .{ }^{20}$ The original work used a push down design as shown in Figure 1-1 in which the PDMS membrane is positioned above the fluidic channel. The valve is normally open, but is closed when the PDMS membrane is pushed down. The second generation of this valve uses the push up layout, which enables lower actuation pressure (from $40 \mathrm{kPa}$ to $15 \mathrm{kPa}$ for the $100 \mu \mathrm{m}$-wide flow channel) and deeper fluid channel depth ( from $\sim 10 \mu \mathrm{m}$ to $55 \mu \mathrm{m}$ ) $\cdot{ }^{19} \mathrm{~A}$ one-layer structure is shown in Figure 1-1. Devices up to seven layers have been developed. ${ }^{13}$ To fabricate multilayered devices, a two-component addition-cure PDMS is used to achieve a hermetic seal between two layers. With this technique, multilayered devices including on-off valves, and switching valves as well as pneumatically actuated pump have been developed. The advantages of this method include rapid prototyping, dead volume as low as $100 \mathrm{pL},{ }^{21}$ easy fabrication and compatibility with biological samples. However, since actuation requires external pneumatic controls, the system requires a network of actuated valves. 


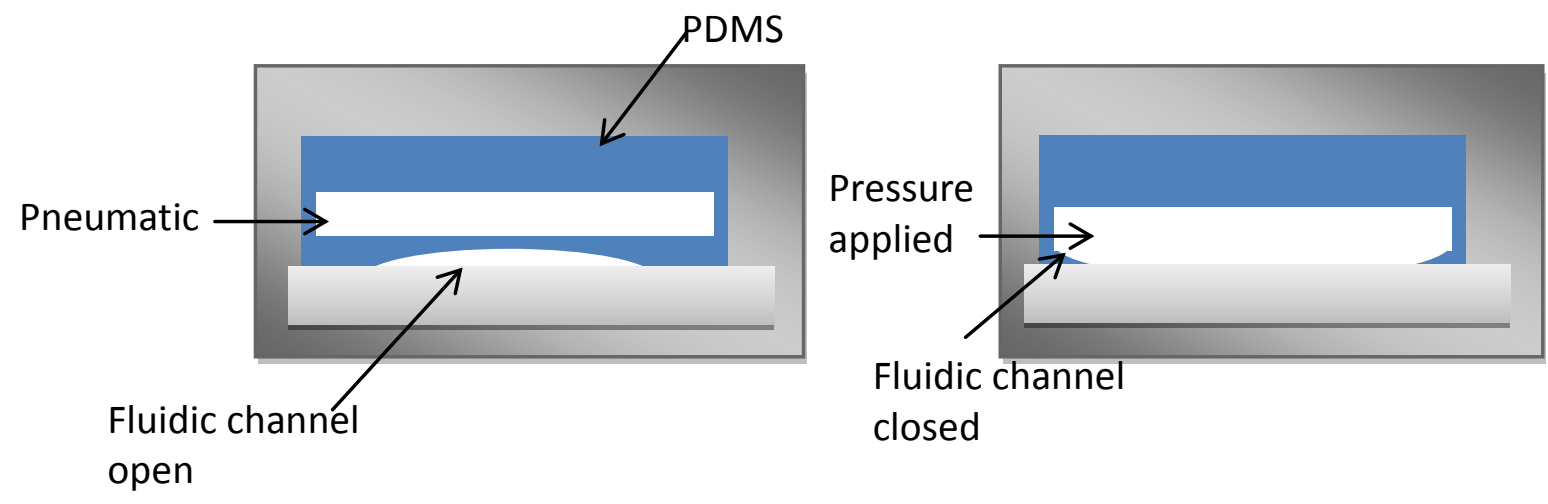

Figure 1-1. Schematics showing the mechanism of the first generation, push down design pneumatic PDMS valve 
The method reported by Quake works for soft elastomeric materials only. The pneumatic valving device suitable for glass chips was developed later. ${ }^{17}$ Glass compatible microfluidic valves are fabricated by sandwiching an elastomer membrane between etched glass fluidic channels. The valves are normally closed. Opening of the valves is actuated by applying vacuum directly to the displacement chambers. Appling a vacuum deflects the PDMS membrane into the displacement chamber, thereby allowing fluid to flow across the fluid channels. These sandwiched valves are suitable for glass chips. However, these pneumatic valves require the design and fabrication to accommodate the displacement chamber in addition to the requirement of external pneumatic controls. A simple approach to realize flow control would be achieved if no extra parts were required in the fluidic channel.

Recently, increasing efforts have been devoted to developing non-mechanical flow control largely due to the simplicity of this approach over traditional mechanical valves. ${ }^{22}$ These non-mechanical microfluidic valves contain no mechanical parts in the fluid channels and work on the basis of volume change of the working fluid in response to an external stimulus. Popular materials used for non-mechanical microvalves include paraffin, ${ }^{18}$ and ice,${ }^{23}$ as well as hydrogel. ${ }^{24} \mathrm{~A}$ concern with hydrogels is that they tend to adsorb proteins. While ice valves pose no contamination to the system, the working temperature is restrictive. Paraffin has a tunable melting point; however, the paraffin plug must be positioned precisely. In addition, once reopened, the paraffin cannot be recovered; therefore the system is only for one-time-use. ${ }^{18}$ 
As has been stated, the goal of this research was to utilize phospholipids for nonmechanical flow control in microfluidic systems. It is important to understand the fundamental properties of flow in the microfluidic channel. Therefore, basic properties regarding mass transfer inside microfluidic channels are reviewed below.

\subsubsection{Fundamental properties of microfluidics}

\subsubsection{Laminar flow}

The small channel diameters of microfluidic devices promote laminar flow. In microchannel laminar flow, the fluid layer in the center has the greatest velocity whereas the fluid layers closest to the microchannel wall have velocities equal to zero. This gives rise to a parabolic flow profile. The opposite of laminar flow is turbulent flow, in which irregular fluctuation or mixing occurs between fluid layers. Laminar flow and turbulent flow in a microchannel are illustrated in Figure 1-2. 

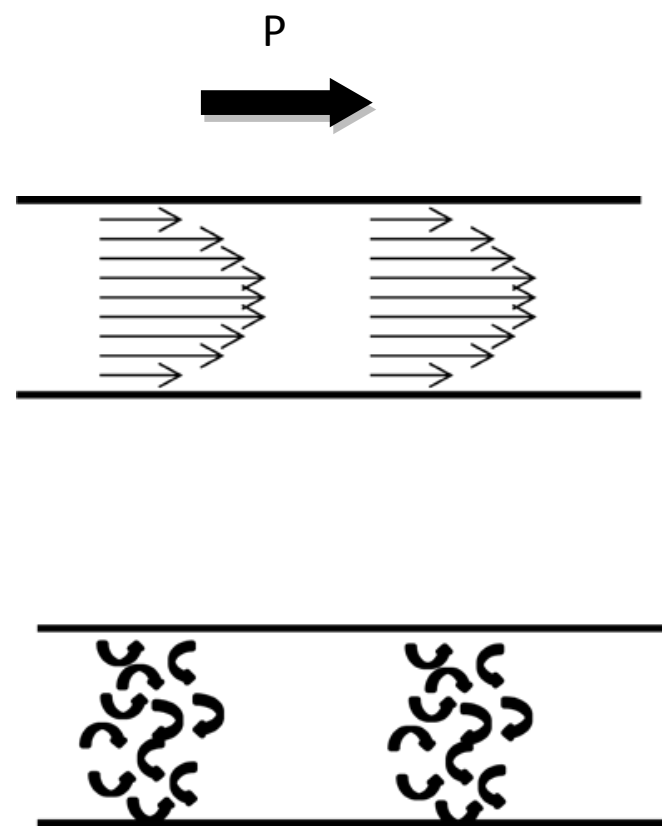

Figure 1-2. Two-dimensional drawings demonstrating the parabolic flow pattern of laminar flow (top) and turbulent flow (bottom) in the microfluidic channel 


\subsubsection{Molecular diffusion in microchannels}

Due to the nature of laminar flow, mixing inside the micro channel is only caused by diffusion. Mixing due to diffusion, which is random thermal molecular motion, becomes more significant in micro fluidic channels as the channel dimensions decrease. The following calculation helps to illustrate this point. Diffusive transport without flow can be estimated from the simplified mass transfer equation. From Fick's second law of diffusion: ${ }^{25}$

$\frac{\partial C}{\partial t}=D \frac{\partial^{2} C}{\partial x^{2}}$

where $\mathrm{C}$ is the concentration, $\mathrm{t}$ is time, $\mathrm{D}$ is the diffusion coefficient and $\mathrm{x}$ is the position. In the beginning the local concentration is assumed to be $C^{*}$. At time $t$, the concentration will be $1 / 2 C^{*}$ at a distance of $\sqrt{\mathrm{Dt}}$, and will be $1 \%$ of $C^{\star}$ at a distance of $4 \sqrt{\mathrm{Dt}}$. Therefore, diffusion has a significant effect up to a distance of $4 \sqrt{\mathrm{Dt}}{ }^{26}$ For example, for small molecules in a fluid, $D$ is on the order of $\sim 10^{-5}-10^{-6} \mathrm{~cm}^{2} / \mathrm{s}$, so for a time period of 1 second, the distance at which diffusion is significant will be in the range of $40-130 \mu \mathrm{m}$, a length that is usually negligible on the macro scale but can be significant in microfluidic systems. Understanding this fact will help the readers to see that hydrodynamic injection in microfluidic chip is hard to implement without additional instrumental design. This is partly because of the non-negligible diffusion of sample inside the fluidic channel. 
1.2.4.3 Enhanced diffusion of pressure driven flow in microchannel (Taylor dispersion)

When a soluble species flows with a pressure driven flow inside a small tube, it spreads out under the combined effect of molecular diffusion and the parabolic flow profile of the laminar flow. The shear flow, or pressure driven flow, which is the flow induced by the applied shearing force, effectively enhances the diffusion of the species in the direction it travels. This shear augmented diffusion is named after G.I. Taylor. ${ }^{19,}{ }^{27,28}$ The broadened peak width, $\mathrm{W}$, is inversely related to the diffusivity, $\mathrm{D}$, of the species in the medium:

$W \propto \sqrt{\frac{v^{2} l^{2}}{D} t}$

Eqn. 1-3

where $v$ is the velocity and I is the diameter of the channel. The diffusivity is inversely related to the viscosity of the medium. Therefore, as the viscosity of the medium increases, the effect of Taylor dispersion becomes more pronounced. Since the goal is to utilize phospholipids as the working fluid for microfluidics and the viscosity of aqueous solutions of phospholipids is greater than water, the band broadening caused by Taylor dispersions effect cannot be neglected. The diagram in Figure 1-3A illustrates the band broadening of an injected sample plug under the combined effect of molecular diffusion and shear flow. An image captured right after an injection plug was made into the channel in phospholipids manifesting the Taylor dispersion is shown in Figure 1-4. 


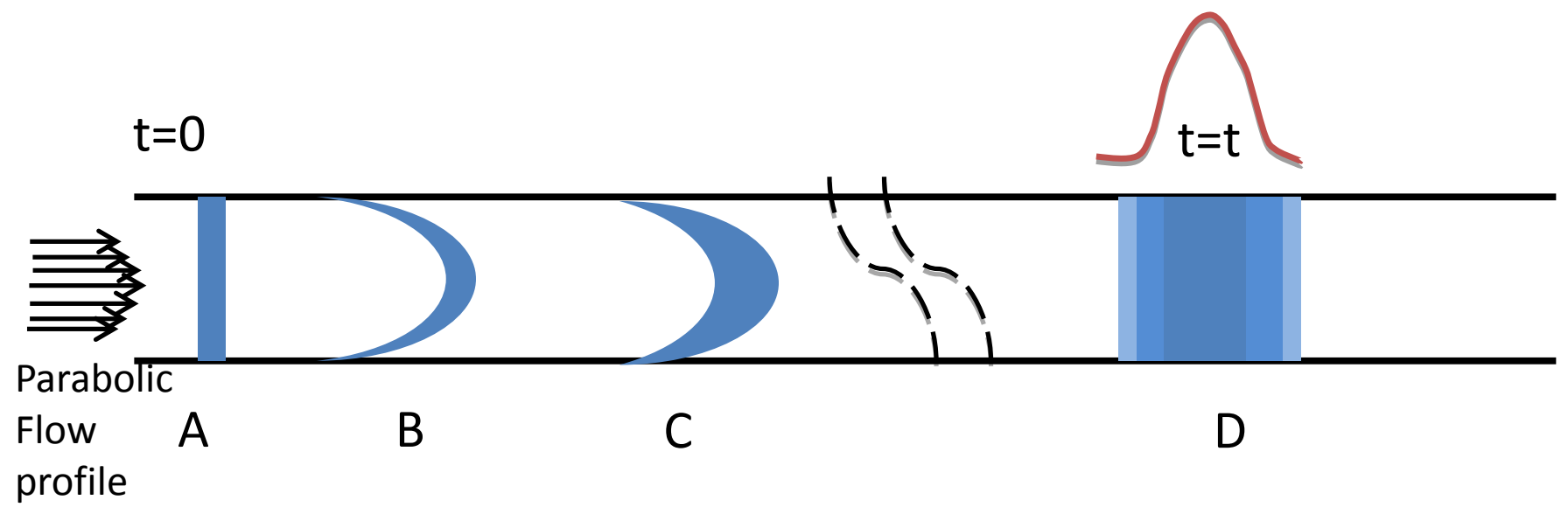

Figure 1-3A.Band broadening resulting from the laminar flow profile and longitudinal diffusion

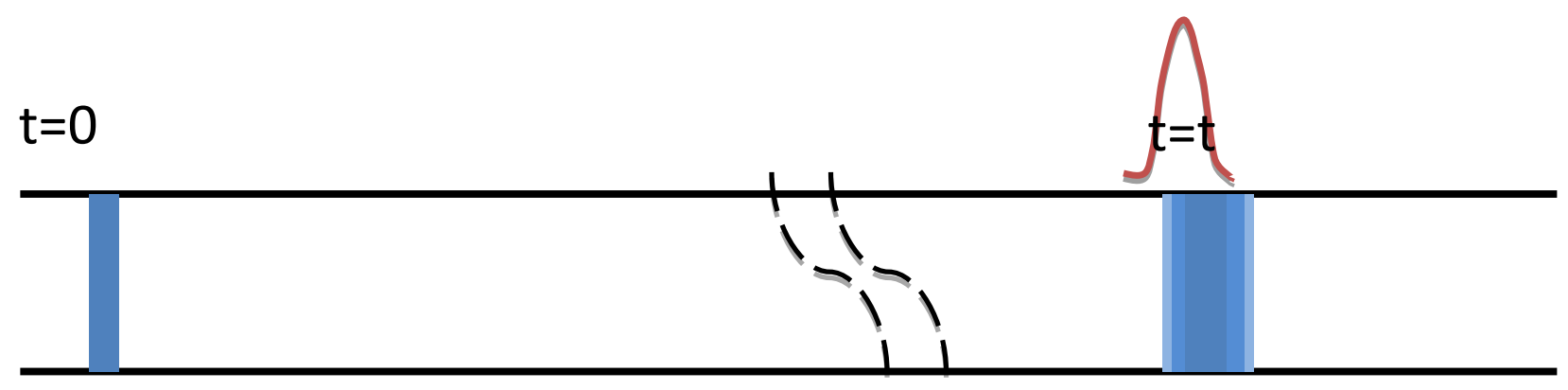

A Figure 1-3B. A hypothetical example of the case in Figure 1-3A if it were possible to obtain data in the absence of laminar flow

In Figure 1-3A, a small sample plug is introduced at time zero (A). Under the influence of shear flow only, the sample plug is dispersed in the direction of the shear (B). A broadening is shown with the finite molecular diffusion (C). After elapsed time $t$, the concentration distribution of the sample plug is Gaussian under the combined effects of molecular diffusion and the shear flow (D). For comparison, Figure 1-3B shows a hypothetical example of the case in Figure 1-3A if it were possible to obtain data in the absence of laminar flow. Figure 1-3 A and B represent how pressure driven flow has shear enhanced diffusion in the direction of the flow. 


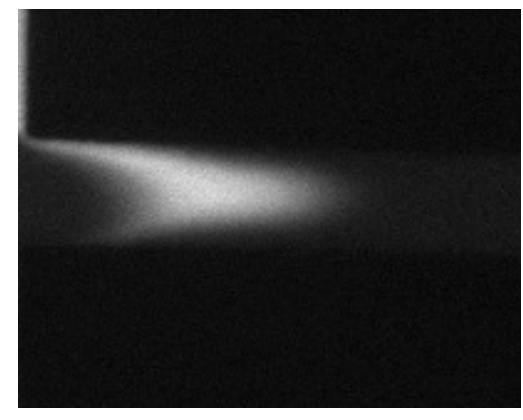

Figure 1-4. Captured fluorescence microscope image showing the Taylor dispersion of the sample plug. The microfluidic channel was filled with clear DMPC-DHPC mixture and fluorescently labeled DMPC-DHPC was used as the sample plug. 


\subsubsection{Practical difficulties of fluid control via direct pressure push}

Intuitively, pressure driven flow would be the most straightforward way to realize fluid control and sample delivery. However, as discussed above, there are limitations posed by the fundamental properties of microfluidics. In addition, other practical issues add difficulties due to flow resistance and mismatched volumes.

\subsubsection{Pressure inside the microchannel}

With viscous liquids such as phospholipids as the working fluid, direct pressure push will result in high pressure inside the microchannel. Example calculations are as follows.

Pressure driven laminar flow through a small tube can be treated using the HagenPoiseuille equation:

$$
\Delta \mathrm{P}=\frac{8 \eta \mathrm{LQ}}{\pi \mathrm{r}^{4}}
$$

where $\mathrm{P}$ is pressure in Pascals, $\eta$ is viscosity in $\mathrm{Pa} \cdot \mathrm{s}, \mathrm{Q}$ is the volume flow rate in $\mathrm{m}^{3} \cdot \mathrm{s}^{-1}$, and $L$ and $r$ are the length and radius of the tube in meters, respectively.

First, assume water is used as the working fluid. The viscosity of water at a temperature of $20^{\circ} \mathrm{C}$ is $0.001 \mathrm{~Pa} \cdot \mathrm{s}$. If the flow rate of the syringe pump is set at $1 \mu \mathrm{L} / \mathrm{min}$, which is representative of what was used in this research, and the channel length is $10 \mathrm{~cm}$, the pressure required to achieve this flow rate is 0.26 bar, or 3.8 psi if the channel radius is $20 \mu \mathrm{m}$. The required pressure is $4.3 \mathrm{bar}$, or $62 \mathrm{psi}$ if the channel radius is $10 \mu \mathrm{m}$.

For fluid control applications, phospholipids are more viscous than water. Although the Hagen-Poiseuille equation applies to Newtonian fluid, it is sufficient for the purpose of 
estimating the pressure. According to the viscosity measurements on chip, the viscosity of phospholipids mixture at $29^{\circ} \mathrm{C}$ is approximately 2000 times greater than that of water ( 2 Pa.s). The pressure required for achieving the flow rate of $1 \mu \mathrm{L} / \mathrm{min}$, for a $20 \mu \mathrm{m}$ radius is 530 bar, or 7690 psi; 8490 bar, or 123,000 psi when the radius is $10 \mu \mathrm{m}$. When $r$ is $10 \mu \mathrm{m}$, even at the flow rate $1 \mathrm{~nL} / \mathrm{min}$, which is the lowest flow rate required of microfluidic applications, the required pressure is still as high as 8.5 bar or 120 psi. The high pressures required to directly pressure push the viscous phospholipid fluid inside the microchannel is impractical. For one thing, the connectors for the chip as well as the chip itself cannot withstand such high pressure; for another, high pressure gases are safety hazards in the laboratory.

\subsubsection{Volume mismatch of the microchannel and the real world connection}

Microfluidic channels are connected to the real world via the connectors glued down to the drilled holes at the terminals of the channels. PEEK (polyetheretherketone) tubing is threaded into each connector to connect to the syringe pump and injection reservoir etc. Currently, there is volume mismatch between the channel and the available connectors. For instance, the volume of the $12 \mathrm{~cm}$ long microchannel used in the project is about 0 . $29 \mathrm{~nL}$, whereas the volume of a piece of $20 \mathrm{~cm}$ long PEEK tubing with an inner diameter of $150 \mu \mathrm{m}$ is $2.1 \mathrm{~nL}$. The connector can hold as much as $40 \mu \mathrm{L}$. With a typical flow rate of $\sim 1 \mu \mathrm{L} / \mathrm{min}$, it is impractical to deliver a small plug into the microchannel in a controlled manner. Syringe pumps capable of flow rates $1 \mathrm{~nL} / \mathrm{min}$ are commercially available, but the time to flush and fill would be unacceptably slow. 
Another consideration is that hydrodynamic flow has a pressure gradient. Thus, the flow cannot be turned on or off instantly. Instead, there is a lag time between the operation of the gas valve and the response of the fluid at the point where the flow control is targeted.

To summarize, although flow control and sample delivery via pressure push is desirable for its simplicity, direct pressure push in microfluidics is not feasible for the fundamental as well as practical reasons outlined above. This research aims to develop a simple, non-mechanical hydrodynamic flow control and sample introduction method by utilizing phospholipids as the working fluid.

\subsection{Phospholipids: a novel responsive material for non-mechanical fluid control}

\subsubsection{Introduction to phospholipids}

Phospholipids are a major component of cell membranes. The properties of phospholipids have been widely studied and applied in the field of analytical chemistry. ${ }^{2-}$ 5, 29-37 In particular, a large number of applications with biological samples have been reported due to the excellent biocompatibility of phospholipids. ${ }^{2-4,29,35}$ Phosphatidylcholines are a class of phospholipids that incorporate choline as a head group. The mixtures of the two phosphatidylcholines 1,2-dimyristoyl-sn-glycero-3phosphocholine (DMPC) and 1,2-dihexanoyl-sn-glycero-3-phosphocholine (DHPC) are the focus of this dissertation. The structures of DMPC and DHPC are shown in Figure 15 and Figure 1-6, respectively. They are amphipathic molecules composed of a 
hydrophilic head group and two hydrophobic alkyl chain tails. DMPC has been found to have a gel-to-liquid crystalline transition temperature of $24{ }^{\circ} \mathrm{C} .{ }^{38}$ In aqueous solutions, self-assembly of the DMPC-DHPC mixture occurs spontaneously in which hydrophilic head groups align facing water molecules and hydrophobic alkyl chains exclude water. This process is energetically favorable. The structures formed have been extensively studied, ${ }^{33-35,39}$ and have been found to be dependent upon the q value (molar ratio of DMPC to DHPC), hydration (weight to volume percentage of lipids), and temperature.

These binary lipid preparations have beneficial properties and various applications have been reported. ${ }^{2-5,30,32,35}$ In capillary electrophoresis, widespread use of DMPC-DHPC preparations has been developed..$^{2-4}$ Phospholipids serve as a semi-permanent coating on glass or fused silica to reduce nonspecific adsorption and suppress electroosmosis flow. ${ }^{3,4}$ DMPC-DHPC mixtures have been used as an alternative media for micellar electrokinetic chromatography ${ }^{30}$ and as additives to achieve enhancement of capillary electrophoresis separations of glycans. ${ }^{3}$ Recently phospholipids have been used as sieving matrix for DNA separation based on size difference. 


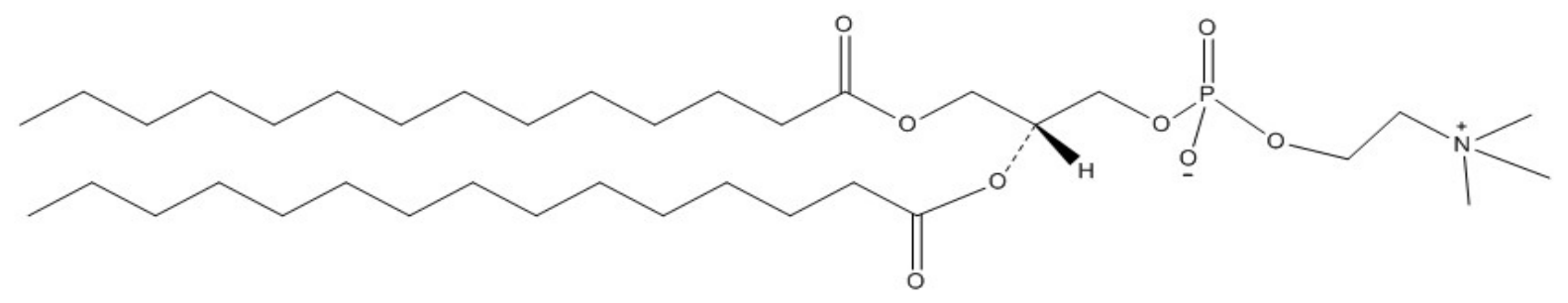

Figure 1-5. Structure of DMPC 


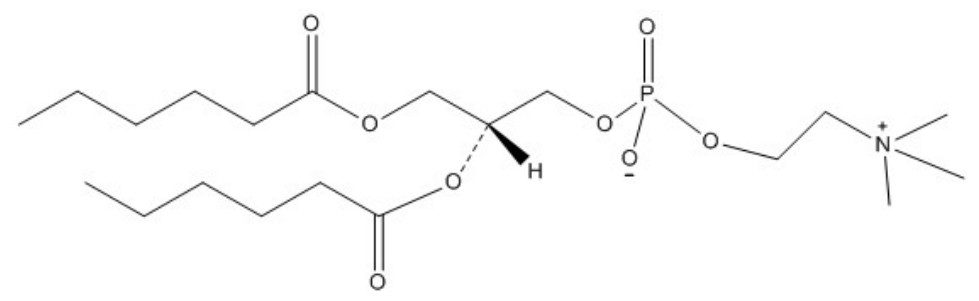

Figure 1-6. Structure of DHPC 


\subsubsection{Studies of DMPC-DHPC properties}

Both microscale and macroscale approaches have been used to study the properties of DMPC-DHPC. Macroscale approach centers on the bulk property of the apparent viscosity and is the focus of this research. Microscale approaches study the individual structures of the assembly. For completeness, microscale approaches are reviewed. On the microscale, extensive studies on the structure and phase behavior of DMPC-DHPC systems have been carried out and various morphologies as well as models have been devised. It is widely accepted that DMPC-DHPC aggregates form discodial aggregates, often called bicelles or nano-disks, with the DMPC bilayer occupying the center, and the DHPC molecules coating on the rim (Figure1-7 a). As temperature increases, the DMPC-DHPC system undergoes phase transition from isotropic to a nematic liquid crystalline phase that displays long range order. More complex morphologies have been described including worm-like shapes (b), ribbons (c), perforated lamellar sheets (d), unilamellar vesicles (e), and perforated multilamellar vesicles (f) as shown in Figure 1-7. Despite the general acceptance of the classical bicelle model, different interpretations have been generated by different methods. ${ }^{38,40-42}$ The fact that studies have been done with lipid preparations of different composition (q value and hydration) and/or at different temperatures may be a source of discrepancy. The following section reviews the microscale approaches including microscopic methods, NMR methods, and scatterings methods. Some other methods such as differential scanning calorimetry have also been used for morphological study of DMPC-DHPC systems. 


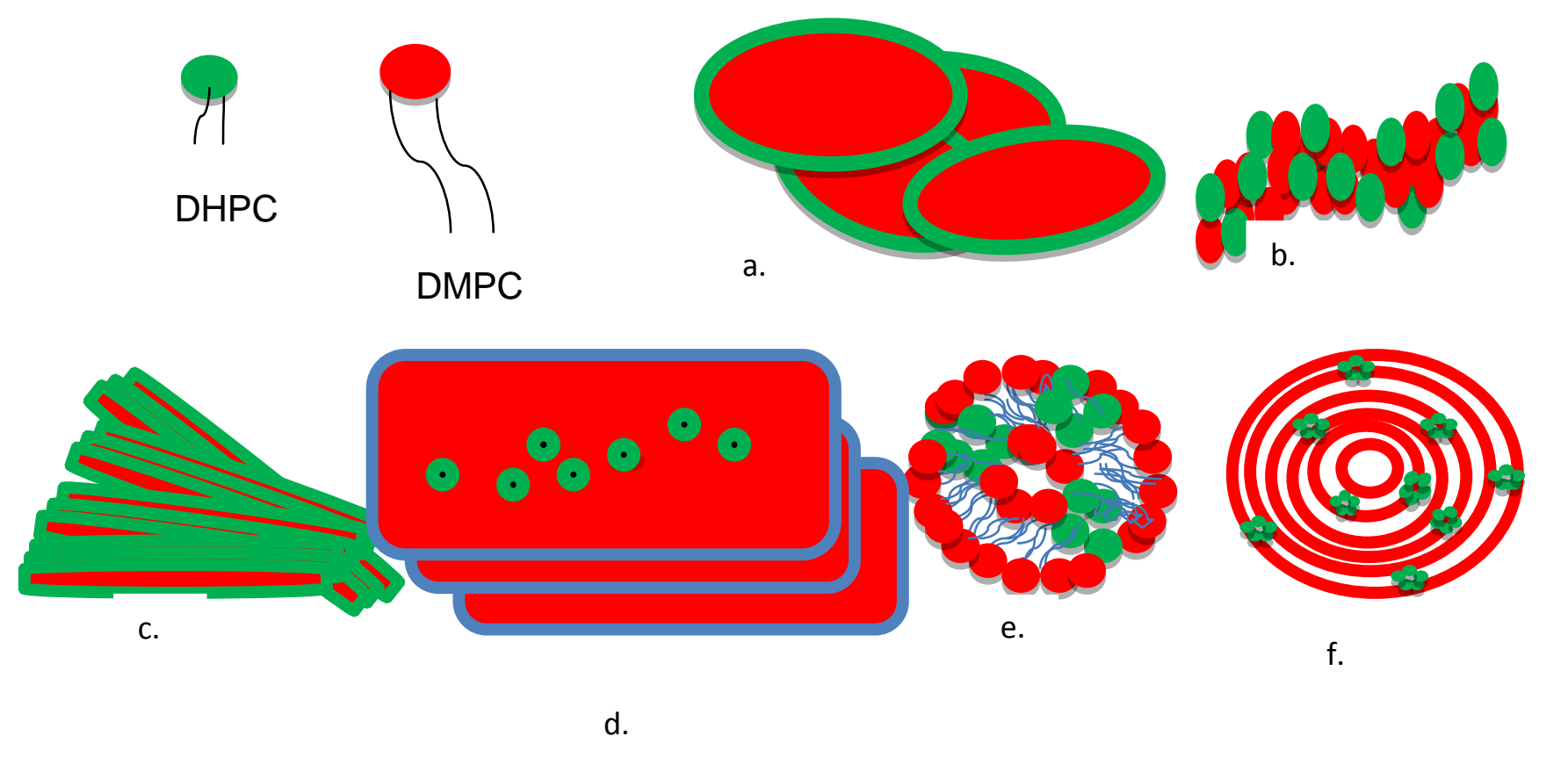

Figure 1-7. Various models of DMPC-DHPC assembly, including the classic bilayered micelle (a), the elongated worm-like shape (b), ribbons (c), perforated lamellar sheets (d), unilamellar vesicle (e), perforated multilamellar vesicle (f). 


\subsubsection{Microscale approaches: morphological study of DMPC-DHPC}

Microscopic methods such as polarized optical microscopy and transmission electron microscopy have been used to directly observe the occurrence of two-phase transitions of DMPC-DHPC preparations. The fingerprint texture was observed as a characteristic of the chiral nematic phase.$^{42}$ However, this feature was not observed in a recent report by Flynn et al. ${ }^{43}$ The application of a confocal microscope, which offers enhanced resolution and holds promise for particle sizing, to observe the morphology of phospholipids has been demonstrated.$^{44}$ Cryogenic transmission electron microscopy was used to directly observe the impact of q value, hydration, and temperature on the morphology of the DMPC-DHPC system. ${ }^{40}$ Results reveal that at $25^{\circ} \mathrm{C}$, as q increases from 2 to 4 , sample morphology changes from roughly all spherical particles (diameter of $7.6 \mathrm{~nm}$ ) to distorted disks, then to elongated and perforated bilayers. ${ }^{40}$ While microscopic methods are more suitable for examining uniform samples or individual samples of interest, a potential concern is that, with very limited area of sample examined, data provided by microscopic methods may be atypical when the long-range alignment and bulk properties of the sample are under investigation.

Scattering techniques, including small-angle neutron scattering (SANS), small-angle Xray scattering (SAXS) and dynamic light scattering (DLS), are classical methods of obtaining information on both size and shape of the assemblies in solution. Complementary SANS and SAXS profiles have been fit to various structure models. The correlation between the scattering intensity and the scattering vector is used to identify certain morphology and to extract size information for phospholipid 
assemblies. ${ }^{40}$ Baohua Yue et al(2005) employed DLS to study spontaneously formed unilamellar vesicles composed of phospholipids. ${ }^{37}$ DLS provides a method to analyze particle size based on the dependence of diffusion coefficient on the partial hydrodynamic radius. It also offers information about the size distribution of the assembly under various conditions. Based on model fitting and mathematical manipulations, however, these scattering methods must meet prerequisites. For example, no interparticle interactions are assumed in DLS. In cases where there is a concentration dependence of the size, the concept of pseudo hydrodynamic radius is used in place of hydrodynamic radius. This limits the use for sample under complex conditions.

Thus far, most information on phospholipid morphology has been obtained with NMR methods. ${ }^{29,38,40,45}$ Deuterium $\left({ }^{2} \mathrm{H}\right)$ labeling of the lipid alkyl chains is an effective measurement of the alignment in the magnetic field because the quadrupolar splitting of the deuterium energy state is orientationally dependent. Information about the line shape of the naturally abundant ${ }^{31} \mathrm{P}$ signal may be indicative of certain morphologies by the relaxation time and thermal motion. In addition, the chemical shift of ${ }^{31} \mathrm{P}$ may indicate the segregation of the different lipids in the mixture. NMR is the predominant method used to study the magnetic alignment of DMPC-DHPC system. Different conclusions have been made regarding the morphology of the magnetic alignable phase; from discoidal shape ${ }^{35}$ to perforated sheets. ${ }^{41}$ Most recently a model has been proposed by Mu-Ping Nieh et al $(2011)^{46}$ which hypothesized that the magnetically alignable smectic phase of lipid aggregates is made up of perforated lamellae and that 
the area fraction of the bilayers occupied by pores decreases with increasing temperature. Effects of divalent cations on DMPC-DHPC bicelle formation and alignment have been investigated. ${ }^{47}$ While NMR methods have been successfully used to extract structural and orientational information of phospholipids under certain conditions, it is difficult to prove the existence of a particular morphology. Moreover, it is impossible with NMR to obtain morphological information in the absence of a magnetic field. X-band electron paramagnetic resonance spectroscopy has also been employed in the study of the magnetic alignment of phospholipids ${ }^{31}$ and it is also subjected to limitations analogous to those of the NMR methods.

Other techniques such as fluorescence spectroscopy and differential scanning calorimetry have also been employed. Fluorescence spectroscopy was used to study the temperature dependence of the concentration of DHPC. ${ }^{40}$ Results show that DHPC monomer solubility is insensitive to temperature in the range of $15^{\circ} \mathrm{C}$ to $45^{\circ} \mathrm{C}$ contrary to what is expected. Differential scanning calorimetry was used to study the morphological effect when DHPC is incorporated into DMPC. ${ }^{36}$ The data show the gel to liquid crystalline transition temperature of DMPC was lowered and the transition temperature range was broadened when incorporated with DHPC.

\subsubsection{Macroscale approach: viscosity behavior of DMPC-DHPC}

As discussed above, from a microscale point of view, DMPC-DHPC assemblies display various structures and undergo phase transitions under different conditions. These 
changes are complex and sensitive to the q value, hydration, temperature, magnetic field and other factors such as salt concentration and presence of cations. Despite a large effort devoted to morphology studies, no consensus has been reached, and these studies are still ongoing.

On the other hand, from a practical point of view, viscosity is a sufficient gauge of fluid behavior. Therefore, an alternative approach is to focus on the study of bulk apparent viscosity. A preliminary viscosity characterization of DMPC-DHPC based on capillary viscometry was performed using a commercial capillary electrophoresis instrument. Results indicated a sharp increase in viscosity from $24^{\circ} \mathrm{C}$ to $29^{\circ} \mathrm{C}$ for the same compositions. By utilizing the viscosity change associated with the morphology change of DMPC-DHPC preparations, a thermally responsive microvalve has been developed previously. ${ }^{5}$

In addition to fluid steering, in capillary electrophoresis separations, the viscosity of the separation media significantly affects the analyte separation as it influences the electrophoretic and electroosmotic mobility in capillary zone electrophoresis. Characterizing and predicting the viscosity of the separation medium is fundamental to enhancing capillary electrophoresis separations. As will be reviewed in more detail in Chapter Two, viscosity characterization of phospholipid systems has been carried out and anomalous viscosity behavior has been reported. ${ }^{5,48}$ However, a systematic viscosity study of the binary DMPC-DHPC system under the same conditions used in the fluid steering is needed in order to best utilize the material. 


\subsection{Multi-purpose use of phospholipids in microfluidic systems}

As an innovative responding material for non-mechanical microvalves in microfluidic devices, phospholipid preparations have attractive benefits such as easy operation and low cost in addition to excellent biocompatibility. Previously, a non-mechanical microvalve was developed in the Holland lab, in which temperature was regulated using

a circulating water bath. ${ }^{5}$ That research demonstrated the feasibility of utilizing phospholipid preparations as a responsive material. However, the response time was limited by the heat dissipation of the configuration. In this research, an improved method of temperature control was achieved through use of a thermoelectric module (TEM). Successful fluid steering, hydrodynamic injection for microchip CE, and electrophoretic separation with DMPC-DHPC preparations have been realized.

The work presented in the following chapters is based on the viscosity characterization of phospholipid DMPC-DHPC preparations under relevant conditions, and centers on using phospholipids as multi-purpose working fluid for microfluidic devices. Chapter Two presents the initial work carried out on viscosity profiling with a custom-made bench-top instrument. This work confirms that phospholipids are a type of power-law fluid and the apparent viscosity is a function of q value, hydration, and temperature. As the study proceeded, the instrumental design was improved by transferring the viscosity characterization to microfluidic chips with better temperature regulation. In Chapter Three, the method, the data processing, and the results of measurement in chip are detailed. Finally, in an attempt to make the microfluidic instrument truly a lab-on-chip instead of a chip-in-lab, a viscosity study of phospholipids under specific relevant 
conditions was carried out. In Chapter Four, the applications achieved using thermally responsive phospholipids for fluid control and sample deliveries as well as capillary electrophoresis separation are described. 


\section{References}

1. Manz, A.; Graber, N.; Widmer, H. M., Miniaturized total chemical analysis systems: a novel concept for chemical sensing. Sensors and Actuators B, 1990; Vol. 1, pp 244248.

2. White, C.; Luo, R.; Archer-Hartmann, S.; Holland, L., Electrophoretic screening of ligands under suppressed EOF with an inert phospholipid coating. Electrophoresis, 2007; Vol. 28, pp 3049-55.

3. Luo, R.; Archer-Hartmann, S. A.; Holland, L. A., Transformable capillary electrophoresis for oligosaccharide separations using phospholipid additives. Anal. Chem., 2010; Vol. 82, pp 1228-1233.

4. Langan, T. J.; Holland, L.A., Capillary electrophoresis coupled to electrospray mass spectrometry through a coaxial sheath flow interface and semi-permanent phospholipid coating for the determination of oligosaccharide labeled with 1aminopyrene-3,6,8-trisulfonic acid. Electrophoresis, 2012; Vol. 33, pp 607-613.

5. Pappas, T. J.; Holland, L. A., Fluid Steering in a Microfluidic Chip by Means of Thermally Responsive Phospholipids. Sensors and Actuators B, 2008; Vol. 128, pp 427-434.

6. Pereira-Rodrigues, N.; Sakai, Y.; Fujii, T., Cell-based microfluidic biochip for the electrochemical real-time monitoring of glucose and oxygen. Sensors and Actuators B, 2008; Vol. 132, pp 608-613.

7. Liu, C. N.; Toriello, N. M.; Mathies, R. A., Multichannel PCR-CE Microdevice for Genetic Analysis. Analytical Chemistry, 2006; Vol. 78, pp 5474-5479.

8. Lai, S.; Wang, S.; Luo, J.; Lee, L.; Yang, S.-T.; Madou, M., Design of a compact disk-like microfluidic platform for enzyme-linked immunosorbent assay. Analytical Chemistry, 2004; Vol. 76, pp 1832-1837.

9. Kaneda, S.; Fujii, T., Integrated microfluidic systems. Advances in Biochemical Engineering/Biotechnology, 2010; Vol. 119, pp 179-194.

10. Jokerst, J.; Emory, J.; Henry, C., Advances in microfluidics for environmental analysis. Analyst, 2012; Vol. 137, pp 24-34.

11. Ha, K.; s Joo, G.; Jha, S. K.; Kim, Y. S., Monitoring of endocrine disruptors by capillary electrophoresis amperometric detector. Microelectronic Engineering, 2009; Vol. 86, pp 1407-1410. 
12. Chin, C.; Linder, V.; Sia, S., Commercialization of microfluidic point-of-care diagnostic devices. Lab-on-A-Chip, 2012; Vol. 12, pp 2118-34.

13. Landers, J. P., editor, Handbook of capillary and microchip electrophoresis and associated microtechniques. CRC Press: Boca Rayton, FL; 2007.

14. Lacher, N. A.; Garrison, K. E.; Scott., M. R.; Lunte, S. M., Microchip capillary electrophoresis/electrochemistry. Electrophoresis, 2001; Vol. 22, pp 2526-2536.

15. Lukacs, K. D.; Jorgenson, J. W., Capillary zone electrophoresis: Effect of physical parameters on separation efficiency and quantitation. Journal of High Resolution Chromatography, 1985; Vol. 8, pp 407-411.

16. Saito, R. M.; Coltro, W. K.; de Jesus, D. P., Instrumentation design for hydrodynamic sample injection in microchip electrophoresis: A review. Electrophoresis, 2012; Vol. 33, pp 2614-2623.

17. Grover, W. H.; Skelley, A. M.; Liu, C. N.; Lagally, E. T.; Mathies, R. A., Monolithic membrane valves and diaphragm pumps for practical large-scale integration into glass microfluidic devices. Sensors and Actuators B, 2003; Vol. 89, pp 315-323.

18. Liu, R. H.; Bonanno, J.; Yang, J.; Lenigk, R.; Grodzinski, P., Single-use, thermally actuated paraffin valves for microfluidic applications. Sensors and Actuators B, 2004; Vol. 98, pp 328-336.

19. Studer, V.; Hang, G.; Pandolfi, A.; Ortiz, M.; Anderson, W. F.; Quake, S. R., Scaling properties of a low-actuation pressure microfluidic valve. Journal of Applied Physics, 2004; Vol. 95, pp 393-398.

20. Unger, M. A.; Chou, H.-P.; Thorsen, T.; Scherer, A.; Quake, S. R., Monolithic microfabricated valves and pumps by multilayer soft lithography. Science, 2000; Vol. 288, pp 113.

21.Zhang, C.; Xing, D.; Li, Y., Micropumps, microvalves, and micromixers with PCR microfluidic chips: Advances and trends. Biotechnology Advances, 2007; Vol. 25, pp 483-514.

22. Pandolfi, A.; Ortiz, M., Improved design of low-pressure fluidic microvalves. Journal of Micromechanics and Microengineering, 2007; Vol. 17, pp 1487-1493.

23. Chen, Z.; Wang, J.; Qian, S.; Bau, H. H., Thermally-actuated, phase change flow control for microfluidic systems. Lab-on-a- Chip, 2005; Vol. 5, pp 1277-1285. 
24. Wang, J.; Chen, Z.; Mauk, M.; Hong, K.-S.; Li, M.; Yang, S.; Bau, H. H., Selfactuated, thermo-responsive hydrogel valves for lab on a chip. Biomedical Microdevices, 2005; Vol. 7, pp 313-22.

25. Fick, A., On Liquid Diffusion. Philosophical Magazine, 1855; Vol.4, No. 10 pp 30-39.

26. Tian, W.-C.; Finehout, E., Microfluidics for biological applications. Springer Science and Business Media, LLC: New York, NY: 2008.

27. Taylor, G., Dispersion of soluble matter in solvent flowing slowly through a tube. Proceedings of the royal society, 1953; Vol. 219, pp 186-203.

28. Aris, R., On the dispersion of a solute in a fluid flowing through a tube. Proceedings of the royal society, 1956; Vol. 235, pp 67-77.

29. Bolze, J.; Fujisawa, T.; Nagao, T.; Norisada, K.; Saito, H.; Naito, A., Small angle Xray scattering and 31P NMR studies on the phases behavior of phospholipid bilayered mixed micelles. Chemical Physics Letters, 2000; Vol. 329, pp 215-220.

30. Holland, L. A.; Leigh, A. M., Bilayered phospholipid micelles and capillary electrophoresis: a new additive for electrokinetic chromatography. Electrophoresis, 2003; Vol. 24, pp 2935-2939.

31. Inbaraj, J. J.; Nusair, N. A.; Lorigan, G. A., Investigating magnetically aligned phospholipid bilayers with EPR spectroscopy at Q-band (35 GHz): optimization and comparison with X-band (9 GHz). Journal of Magnetic Resonance, 2004; Vol. 171, pp 71-79.

32. Prosser, R. S.; Hwang, J. S.; Vold, R. R., Magnetically aligned phospholipid bilayers with positive ordering: a new model membrane system. Biophysical journal, 1998; Vol. 74, pp 2405-2418.

33. Ram, P.; Prestegard, J., Magnetic field induced ordering of bile salt/phospholipid micelles: a new media for NMR structural investigations. Biochimica et Biophysica Acta (BBA)-Biomembranes, 1988; Vol. 940, pp 289-294.

34. Sanders, C. R.; Prestegard, J. H., Magnetically orientable phospholipid bilayers containing small amount of a bile salt analogue, CHAPSO. Biophysical Journal, 1990; Vol. 58, pp 447-592.

35. Sanders, C. R.; Hare, B. J.; Howard, K. P.; Prestegard, J. H., Magnetically-oriented phospholipid micelles as a tool for the study of membrane-associated molecules.

Progress in Nuclear Magnetic Resonance Spectroscopy, 1994; Vol. 26, pp 421-444. 
36. Takajo, Y.; Matsuki, H.; Matsubara, H.; Tsuchiya, K.; Aratono, M.; Yamanaka, M., Structural and morphological transition of long-chain phospholipid vesicles induced by mixing with short-chain phospholipid. Colloids and Surfaces B: Biointerfaces, 2010; Vol. 76, pp 571-576.

37. Yue, B.; Huang, C.-Y.; Nieh, M.-P.; Glinka, C. J.; Katsaras, J., Highly stable phospholipid unilamellar vesicles from spontaneous vesiculation: a DLS and SANS study. Journal of Physical Chemistry. B, 2005; Vol. 109, pp 609-616.

38. Sternin, E.; Nizza, D.; Gawrisch, K., Temperature dependence of DMPC/DHPC mixing in a bicellar solution and its structural impllications. Langmuir, 2001; Vol. 17, pp 2610-2616.

39. Sanders, C. R.; Schwonek, J. P., Characterization of magnetically orientable bilayers in mixtures of dihexanoylphosphatidylcholine and dimyristoylphosphatidylcholine by solid-state NMR. Biochemistry, 1992; Vol. 31, pp 8898-8905.

40. Dam, L. V.; Karlsson, G.; Edwards, K., Direct observation and characterization of DMPC/DHPC aggregates under conditions relevant for biological solution NMR. Biochimica et Biophysica Acta (BBA)-Biomembranes, 2004; Vol. 1664, pp 241-256.

41. Dam, L. v.; Karlsson, G.; Edwards, K., Morphology of magnetically aligning DMPC/DHPC aggregates-perforated sheets, not disks. Langmuir, 2006; Vol. 22, pp 3280-3285.

42. Harroun, T. A.; Koslowsky, M.; Nieh, M.-P.; Lannoy, d. C.-F.; Raghunathan, V. A.; Katsaras, J., Comprehensive examination of mesophases formed by DMPC and DHPC mixtures. Langmuir, 2005; Vol. 21, pp 5356-5361.

43. Flynn, A.; Ducey, M.; Yethiraj, A.; Morrow, M. R., Dynamic properties of bicellar lipid mixtures observed by rheometry and quadrupole echo decay. Langmuir, 2011; Vol. 28, pp 2307-3062.

44. Korlach, J.; Schwille, P.; Webb, W. W.; Feigenson, G. W., Characterization of lipid bilayer phases by confocal microscopyand fluorescence correlation spectroscopy. Proceedings of the National Academy of Science, 1999; Vol. 96, pp 8461-8466.

45. Dvinskikh, S.; Durr, U.; Yamamoto, K.; Ramamoorthy, A., A high resolution solid state NMR approach for the structural studies of bicellles. Journal of the American Chemical Society, 2006; Vol. 128, pp 6326-6327.

46. Nieh, M.-P.; Raghunathan, V. A.; Pabst, G.; Harroun, T.; Nagashima, K.; Morales, $\mathrm{H}$.; Katsaras; Macdonald, P., Temperature driven annealing of perforations in bicellar model membranes. Langmuir, 2011; Vol. 27, pp 4838-4847. 
47. Brindley, A. J.; Martin, R. W., Effect of divalent cations on DMPC/DHPC bicelle formation and alignment. Langmuir, 2012; Vol. 28, pp 7788-7796.

48. Hwang, J. S.; Oweimreen, G. A., Anomalous viscosity behavior of a bicelle system with various molar ratios of short- and long- chain phospholipid. The Arabian Journal for Science and Engineering, 2003; Vol. 28, pp 43-49. 
CHAPTER 2

Viscosity Characterization of DMPC-DHPC

Phospholipid Preparations in Circular Ducts 


\subsection{Introduction: viscosity of phospholipid preparation}

Phospholipid preparations comprised of 1,2-dimyristoyl-sn-glycero-3-phosphocholine (DMPC) and 1,2-dihexanoyl-sn-glycero-3-phosphocholine (DHPC), which possess an unusual viscosity profile,$^{1-3}$ have been utilized as running buffer additives in capillary electrophoresis to significantly enhance the performance of capillary separation. The viscosity of a phospholipid preparation is dependent on the ratio of the long-chain and short-chain lipids, the lipid concentration, and the temperature. Below temperatures of $24^{\circ} \mathrm{C}$, the material is thought to form bilayered discs called bicelles or nanodiscs. Above this temperature, the viscosity can increase dramatically. Phospholipid preparations self-assemble into a variety of morphologies, including wormlike micelles. ${ }^{4}$,

${ }^{5}$ In a separation capillary, the elongated micelles can entangle in a manner analogous to polymers and increase dramatically in viscosity. Rheological characterization of wormlike micelles is intriguing but also critical to harnessing the power of these materials. ${ }^{6}$ The morphology of phospholipid preparations has been investigated, ${ }^{7-9}$ but the characterization of viscosity has been limited. ${ }^{10-12}$ In addition to applications in microscale chemical separations, ${ }^{1-3}$ these materials have significant applications in solid-state $\mathrm{NMR}^{13}$ and templating,${ }^{14}$ as well as fluid steering. ${ }^{12} \mathrm{~A}$ clear understanding of viscosity is critical to expanding applications that incorporate the material to enhance or facilitate chemical separations. This chapter outlines the methodology used to verify that phospholipid preparations are shear-thinning power-law fluids. For the first time, the apparent viscosity is determined in microscale channels. The apparent viscosity is affected by shear rate, temperature, the amount of aqueous diluents, and channel geometry. 


\subsubsection{The importance of viscosity}

In capillary electrophoresis separations, the viscosity of the separation media significantly affects the analyte separation, because it influences the electrophoretic and electroosmotic mobility in capillary zone electrophoresis. For capillary gel separations with linear polymers, the viscosity of the medium is related to the apparent pore size, which in turn affects the size-based sieving of biopolymers. Characterizing and predicting the viscosity of the separation medium is fundamental to enhancing capillary electrophoresis separations. In capillary electrophoresis, the velocity of the analyte is defined as:

$\mathrm{V}=\mu_{\mathrm{eph}} \mathrm{E}$

where $E$ is the field strength of the electric field. The electrophoretic mobility, $\mu_{\mathrm{eph}}$, is defined as:

$\mu_{\text {eph }}=\frac{q}{6 \pi n r}$

where $q$ is the charge on the particle, $r$ is the Stokes radius and $\eta$ is the viscosity of the background electrolyte.$^{15}$ Temperature tunable viscosity behavior of phospholipids offers tunable viscosity to adjust the electrophoretic mobility of samples by controlling the viscosity of background electrolyte to achieve desired separation. In a recent application in capillary electrophoresis, DMPC-DHPC was added to the background electrolyte to improve glycan separations. ${ }^{16}$ 


\subsubsection{Definition of viscosity and non-Newtonian fluid}

Viscosity is a property that describes the resistance of a material to the shear force applied. Therefore, viscosity $\eta$ can be characterized as:

$$
\eta=T / Y
$$

where $\mathrm{T}$ is the shear force and $\mathrm{Y}$ is the shear rate.

Most liquids have a constant value of viscosity at a given temperature. In fact, viscosity is the constant proportionality between shear stress and shear rate. Such fluids are named Newtonian fluids in honor of Sir Isaac Newton. Newtonian viscosity therefore is a point value, and only the temperature of the measurement needs to be stated. Other fluids have a viscosity that varies with shear rate. In these special cases fluids are called non-Newtonian fluids. The behavior of non-Newtonian fluids can be complicated. Numerous attempts have been made to approximate the real fluid properties using simple analytical functions. The Ostwald-de Waele model or Power-law model ${ }^{19}$ is commonly used. A Power-law fluid is an idealized fluid for which the shear stress, $\tau$, is given by:

$\tau=K \gamma^{n}$ Eqn. 2-4

where $\mathrm{K}$ is the flow consistency index ( $\mathrm{SI}$ units $\mathrm{Pa} \cdot \mathrm{s}^{\mathrm{n}}$ ), and $n$ is the flow behavior index (dimensionless). Power-law fluids can be subdivided into three different types of fluids based on the value of their flow behavior index $n$ : shear thinning with $n$ less than 1 ; shear thickening with $n$ greater than 1 and Newtonian fluid when $n$ is equal to unity. 


\subsubsection{Viscosity characterization of non-Newtonian fluid}

Various methods have been developed for viscosity measurements. They are all based on the relationship of shear force and shear rate. Generally, shear viscometers can be categorized as pressure driven flow or as drag flow, based on how the shear is generated. For pressure driven flow, shear is generated by a pressure difference over a channel. For drag flow, shear is generated between a moving and a fixed solid surface. Viscometers based on pressure driven shear include the capillary viscometer, the slit viscometer and axial annulus flow. Examples of drag flow viscometers include sliding plates, falling ball, cone-and-plate, and parallel disks. Among these methods, the capillary viscometer is the most suitable for viscosity measurement of phospholipids.

\subsubsection{Capillary viscometer}

The capillary viscometer is the oldest viscometer. In 1839, Hagen in Germany and in 1840 Poiseuille in France independently used capillary tubes to measure the viscosity of water. Capillary viscometery still remains the most widely used method for several reasons. The capillary viscometer is simple, relatively inexpensive, easy to construct and customize, and the measurement result is accurate for steady viscosity flow. The system is easy to pressurize and as a closed system it is not subject to evaporation. With small diameter capillary tubes, sample consumption is minimal. Different inner diameter capillaries can be utilized to study the wall slip effect of the material. A great advantage to use of capillary viscometer for phospholipids measurement is that it is capable of a wide range of shear rates. In fact, for viscous polymer melts, capillary or slit viscometers are the only satisfactory means of obtaining data at shear rates greater 
than $10 \mathrm{~s}^{-1} \cdot{ }^{20}$ In addition, it better reflects the flow behavior for pressure driven flow in a microchannel.

2.1.3.2 Measurement of apparent viscosity of phospholipids with capillary viscometer Viscosity measurement of Newtonian fluids by capillary viscometry is based on the Hagen-Poiseuille equation. At a controlled temperature, the elution time of an analyte through a capillary tube is a function of pressure, radius, and the length of the capillary. As phospholipids have been found to be power law fluids, measurements need to be made at different pressures to determine shear stress at different values of shear rate. A velocity measurement at a specific pressure is made in triplicate. To determine the apparent viscosity, a capillary is filled with the phospholipid preparation under investigation. A plug of the phospholipid preparation doped with fluorescein isothiocyanate (FITC) is introduced into a capillary and pushed to the detection window at a known temperature and applied pressure. The model for pressure driven flow in capillary is shown in Figure 2-1. The shear stress, $\tau$, is calculated from the following equation:

$\tau=\mathrm{D} \Delta \mathrm{P} / 4 \mathrm{~L}$ Eqn. $2-5$ where $\mathrm{D}$ is the inner diameter of the capillary, $\Delta \mathrm{P}$ is the applied pressure (in $\mathrm{Pa}$ ), and $\mathrm{L}$ is the tube length. The estimated shear rate, $\gamma_{\mathrm{e}}$, is estimated using the relationship:

$\gamma_{\mathrm{e}}=8 \mathrm{~V} / \mathrm{D}$ 
where $\mathrm{V}$ is the average linear velocity of the measured plug taken by dividing the length to the detection window by the elution time of the fluorescently labeled plug. The shear stress and the estimated shear rate are fit to the equation:

$\tau=K^{\prime} \gamma^{n}$

Eqn. 2-7

From this equation, the power-law index $n$ and uncorrected consistency index $\mathrm{K}^{\prime}$ is

determined. The power-law index $n$ is used to adjust the estimated shear rate using the Rabinowitsch-Mooney(R-M) correction: ${ }^{20}$

$\gamma=(3 / 4+1 / n) \gamma_{\mathrm{e}}$

Eqn. 2-8

Each data set is fit to the equation using nonlinear regression to calculate the corrected consistency index, $\mathrm{K}$ :

$\tau=\mathrm{K} \gamma^{n}$

Eqn. 2-9

The power-law parameters $\mathrm{K}$ and $n$ can be obtained accordingly. The apparent viscosity is written:

$\eta_{\mathrm{A}}=\mathrm{K} \gamma^{(n-1)}$

Eqn. 2-10

The apparent viscosity is then determined using Equation 2-10. A step-by-step calculation from the measured elution time to the apparent viscosity is shown in section 3.3.1. Typically the relationship between viscosity and shear rate is displayed as a log-log plot as shown in Figure 2-8. In this figure, the non-Newtonian (i.e. sheer-thinning power-law) fluid varies in viscosity over a wide range of shear rate. Fluids that display Newtonian behavior over the shear range reported yield a straight-line with a slope equal to zero. 


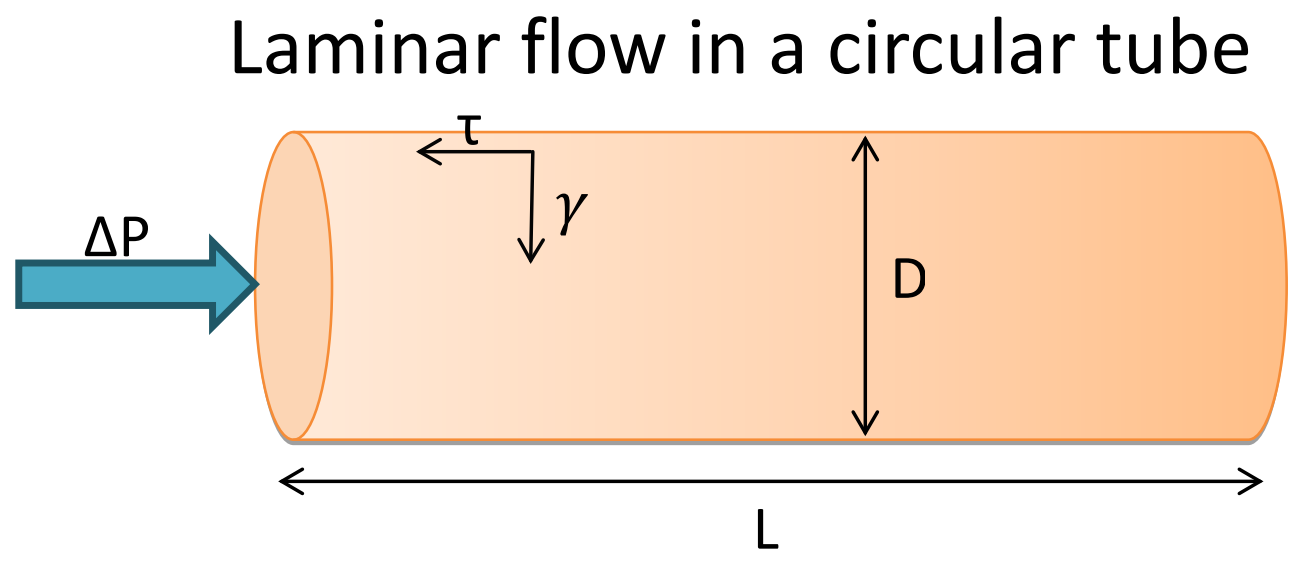

Figure 2-1. A schematic of pressure driven flow in a circular tube 


\subsubsection{Cone-and-plate viscometer}

The basic geometry of the cone-and plate viscometer is consisted of an inverted rotating cone and a flat plate. The angle between the cone and the plate is usually very

small. A schematic of cone-and-plate viscometer is shown in Figure 2-2. Fluid sample to be measured is placed in the gap between the cone and the plate. When a defined shear rate (from the set angular velocity) is applied, the torque on the plate, caused by the resistance to the rotation, is measured. The measured torque can be directly translated into shear stress, which is plotted against the shear rate to yield the apparent viscosity.

Unlike a capillary viscometer which measures shear rate at a single shear stress value at a time, with a cone-and-plate viscometer, discrete shear rates can be set to take the measurement quickly. The cone-and-plate viscometer is a popular method to measure non-Newtonian fluids, because they require multiple shear rates. However, there are limitations. A cone-and-plate viscometer works based on the assumption that when the angle between the cone and plate as well as the shear rate is sufficiently small, the shear rate and shear stress are uniform throughout the flow. Errors arise when the angular velocity increases, a secondary flow develops due to centripetal acceleration. ${ }^{16}$ One report concluded that effect of secondary flow is more significant for shear thinning $(n<1)$ non-Newtonian fluid than shear thickening fluid $(n>1) \cdot{ }^{20}$ In addition, the sample may be susceptible to evaporation and the small angle plate requires accurate alignment. Compared to a capillary viscometer, the cone-and-plate viscometer is incapable of studying the wall-slip effect or the turn effect in a channel. Overall, a cone- 
and-plate viscometer is useful to provide a quick measurement in the regions where shear rate is not too high, while a capillary viscometer is capable of obtaining more meaningful information at the cost of time. For example, in the initial viscosity study, the general trend of data obtained with bench top instrument does not match with the reported data measured with cone-and-plate viscometer. ${ }^{22}$ In that paper, cone-andplate viscometer was used to measure the viscosity of DMPC-DHPC phospholipids mixture of $q=3,4$ and 5 and hydration approximately $10 \%$. The data reported show that for $\mathrm{q}=5$ lipids mixture, the flow behavior $n$ is unity below $20^{\circ} \mathrm{C}$ and $n$ is smaller than 1 at temperatures above $20^{\circ} \mathrm{C}$. However, the measured flow behavior index $n$ from the bench-top study increases from 0.52 to 0.89 as the temperature increases from 20 to 29 ${ }^{\circ} \mathrm{C}$, indicating the phospholipids mixture is more shear thinning at $20^{\circ} \mathrm{C}$. Although lipid mixtures of different q values were measured in our study, the discrepancy of the general trend of $n$ still reminded us to check for instrumental flaws. The viscosity measurement based on capillary viscometry was therefore transferred to microchip to overcome such flaws to yield more meaningful information. 


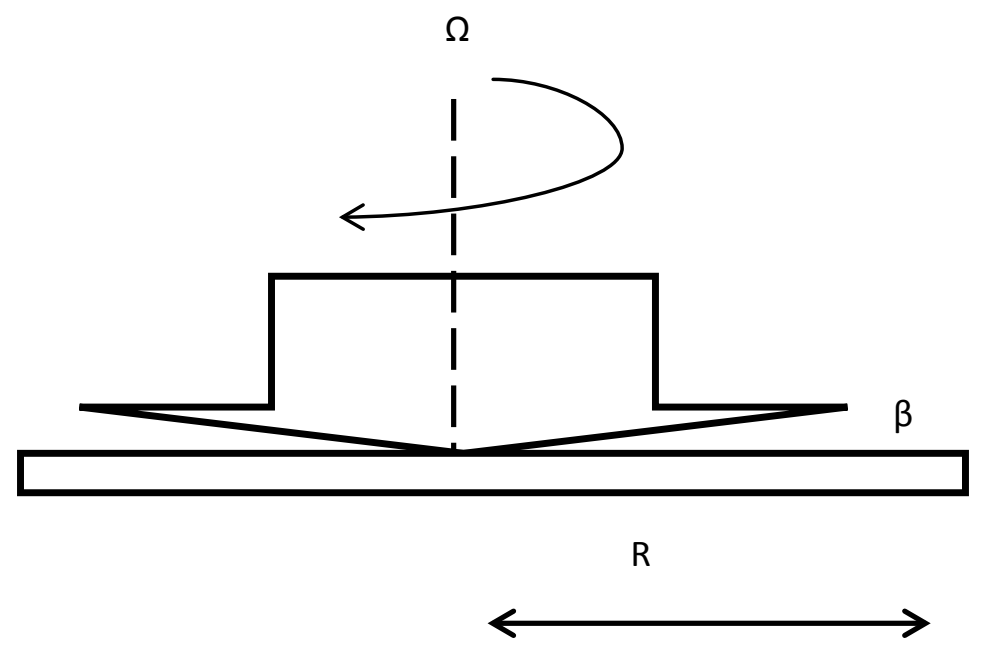

Figure 2-2. A schematic of a cone-and-plate viscometer 


\subsubsection{Custom built system for viscosity measurement of phopsholipids}

Viscosity measurements of phospholipid preparations have previously been performed using a Beckman PIACE MDQ capillary electrophoresis system equipped with a UVvisible absorbance detector (Beckman Coulter, Fullerton, CA, USA) ${ }^{12}$ These measurements were made in a $75 \mu \mathrm{m}$ inner diameter capillary, $40 \mathrm{~cm}$ long, and $32.8 \mathrm{~cm}$ to the window using UV-visible absorbance detection at wavelength of $200 \mathrm{~nm}$. In that study, phospholipid preparations demonstrated up to 425 -fold increase in viscosity with $9 \mathrm{C}^{\circ}$ increase in temperature. There are limitations to the use of the commercial instruments. For instance, the commercial instrument limits the maximum applied pressure to $1.4 \times 10^{5} \mathrm{~Pa}(20 \mathrm{psi})$ and it limits the run times to 180 minutes. Therefore, in order to profile the viscosity of the phospholipid as a function of pressure, a custom built pressure system was used. The custom system could be pressurized up to $100 \mathrm{psi}$ and retention times of up to two days were recorded.

The custom built instrument consists of a stainless steel reservoir for hydrodynamic injection, a fused silica capillary inserted in Teflon tubing in which water from a circulating water bath is running, a UV visible absorbance detector to detect the hydrodynamic elution time, and a gas tank filled with helium or nitrogen gas. Measurements were performed simultaneously in triplicate using three capillaries with an inner diameter of $100 \mu \mathrm{m}$ or $50 \mu \mathrm{m}$. The capillaries were thermally regulated by three different water baths (Isotemp 3016, Fisher Scientific, PA, USA). The pressure reservoir was thermally regulated by a thermoelectric module (TEM) (FerroTec, NH, USA) controlled by a FTC100 controller (FerroTec, NH, USA). A labeled image of the 
instrumentation is shown in Figure 2-3. Figure 2-4 is a close up view of the setup showing the temperature control methods. Figure $2-5$ is a diagram of the custom built pressure reservoir that enables simultaneous triplicate measurements. Each morning the capillary was flushed with water, 3-(N-morpholino)propanesulfonic acid (MOPS), and then the phospholipid preparation. The velocity was observed by injecting a plug of fluorescently doped phospholipid. The fluorescently doped phospholipid was pressure injected into the capillary, and the elution time of the sample plug was determined at a specific temperature and applied pressure. The injected fluorescently doped phospholipid plug was detected by UV-visible absorbance detectors. 


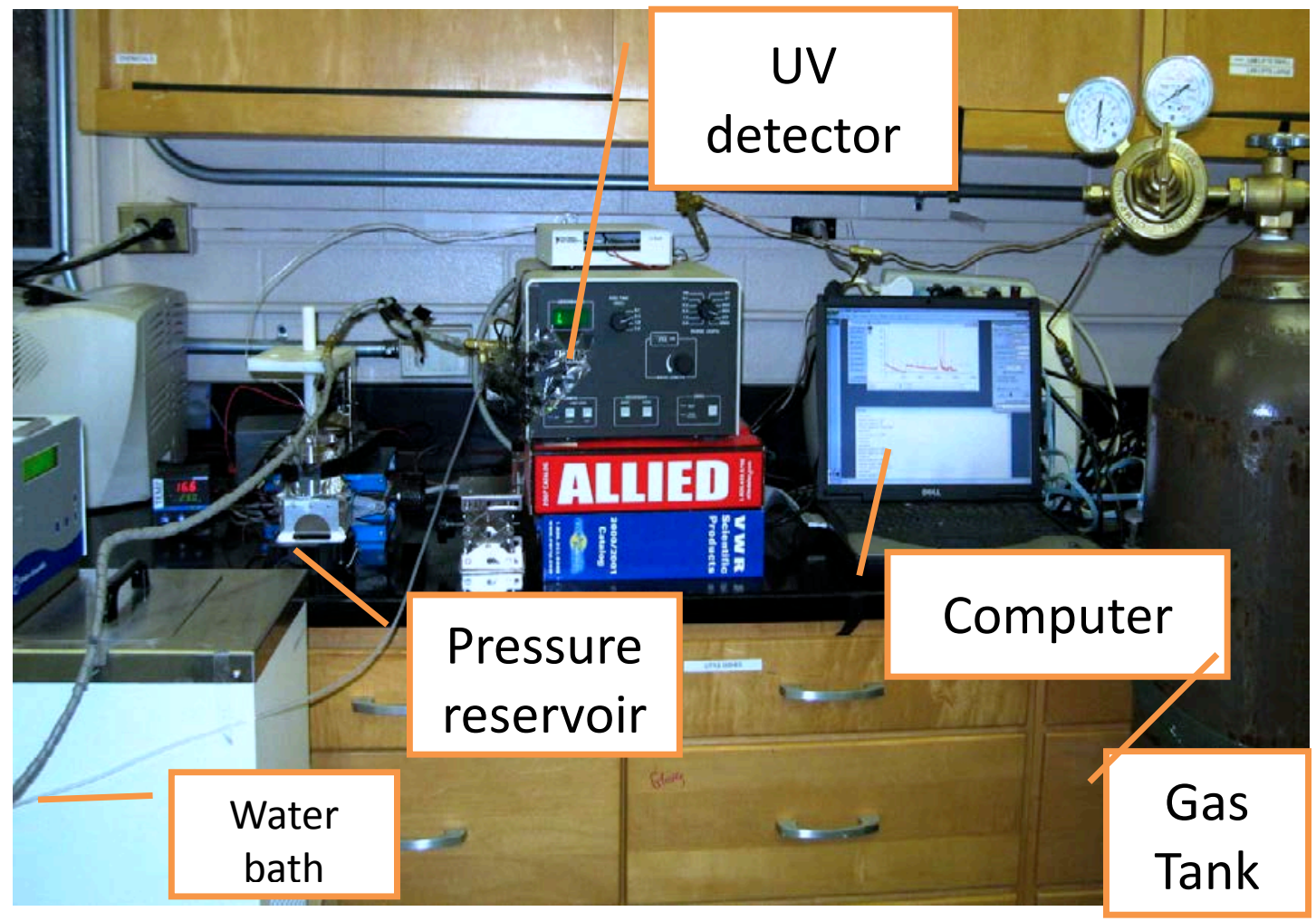

Figure 2-3. A photograph showing the components of the custom-built pressure system. 


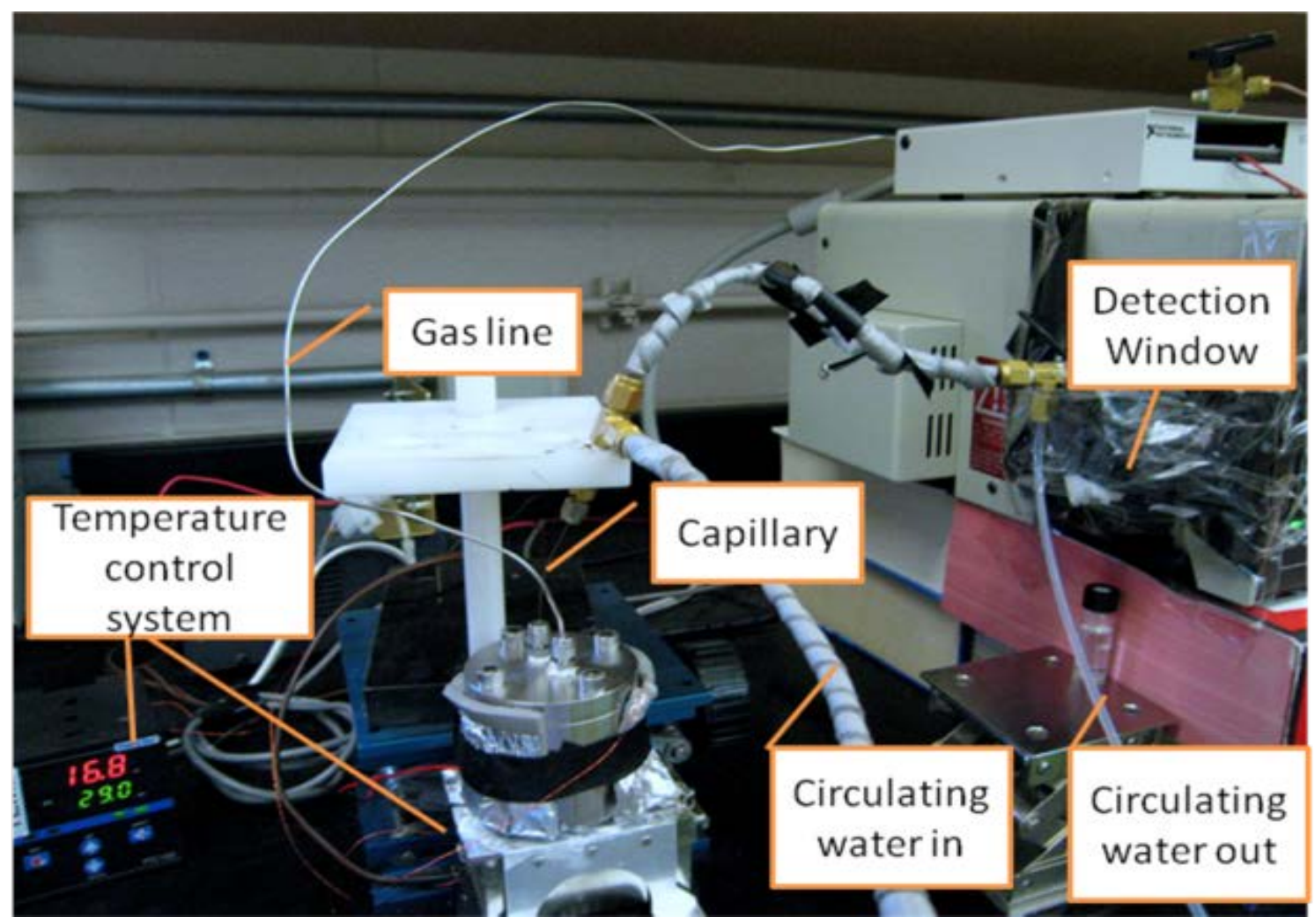

Figure 2-4. A picture of the custom made pressure system. A sample vial filled with phospholipids was placed inside the pressure reservoir. The pressure reservoir was connected to the gas tank via copper tubing. The pressure range of the gauge was 10-100 psi. The fused silica capillary connected to the pressure reservoir was a total of $90 \mathrm{~cm}$ in length and it was $9 \mathrm{~cm}$ from the detection window to the end. The capillary was inserted into Teflon tubing that connects to the circulating water bath. The temperature of the pressure reservoir was controlled by a TEM system. The UV detector was set at wavelength $254 \mathrm{~nm}$. 


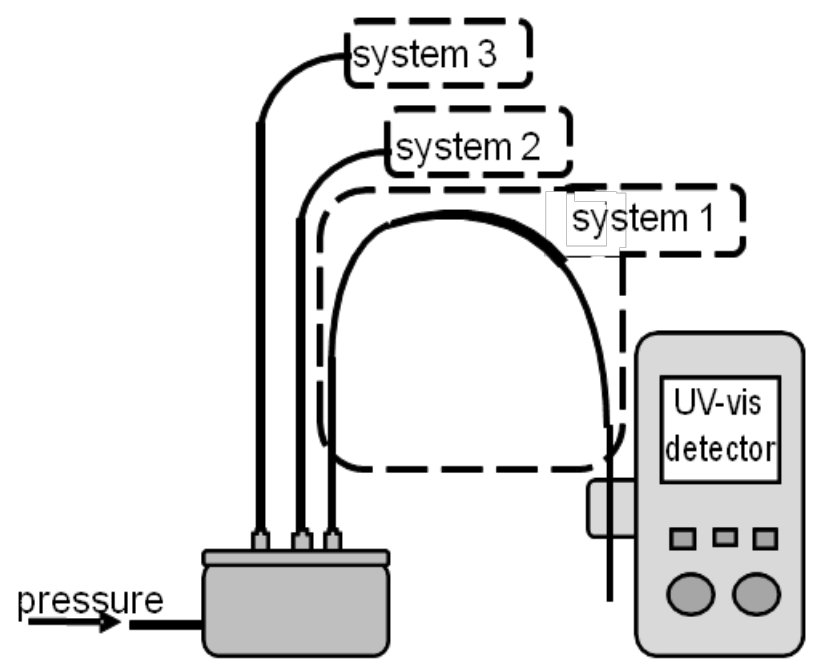
A. Schematic of the instrumental setup for the bench-top viscosity

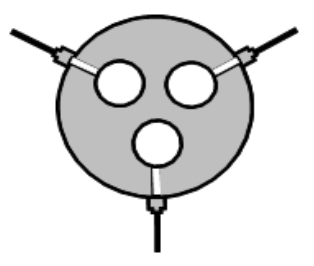

B. Cross-section view of the pressure reservoir

Figure 2-5A contains a schematic of the custom-built pressure reservoir that allows simultaneous measurement of three sets of capillary measurements using a single gas cylinder. A cross sectional view is shown in shown in $B$. 


\subsubsection{Temperature control with thermoelectric module (TEM)}

A thermoelectric module, also called a thermoelectric cooler or Peltier cooler, is a semiconductor-based electronic device with ceramic substrate (as shown in Figure 2-6) that functions as a small heat pump. The direction of the heat flow is reversible depending on the polarity of the current. The TEMs used in this work range in size from approximately $2.5-50 \mathrm{~mm}$ (0.1 to 2.0 inches) square and $2.5-5 \mathrm{~mm}$ (0.1 to 0.2 inches) in height. Each flat TEM is placed in contact with the object to be temperature regulated. The thermistor is placed in contact with the object. A thin layer of thermal paste (Type 79 Heat Sink Compound, GC Electronics, Rockford, IL) is applied in between the object surface and the TEM or the thermistor to assist in the heat transfer. The thermistor and TEM are connected to an electronic controller so that the TEM reaches a set temperature based upon feedback from the temperature sensor. As shown in Figure 27, a TEM temperature control system consists of a low DC voltage supply, an amplifier board (FTX700D H-Bridge Amplifier, Accuthermo Technology Corp. Fremont, CA, USA), a controller (FTC 100D TEC, Accuthermo Technology Corp. Fremont, CA , USA) and a thermistor (LSMN-TR2252, Accuthermo Technology Corp. Fremont, CA , USA). The TEMs are a portable alternative to water baths, but more importantly, the temperature regulation achieved with TEMs is more accurate, precise, and reproducible and faster temperature switching can be readily achieved. TEM system can be controlled and multi-step programmed with a computer via FTC100 software (Accuthermo Technology Corp. Fremont, CA, USA). 


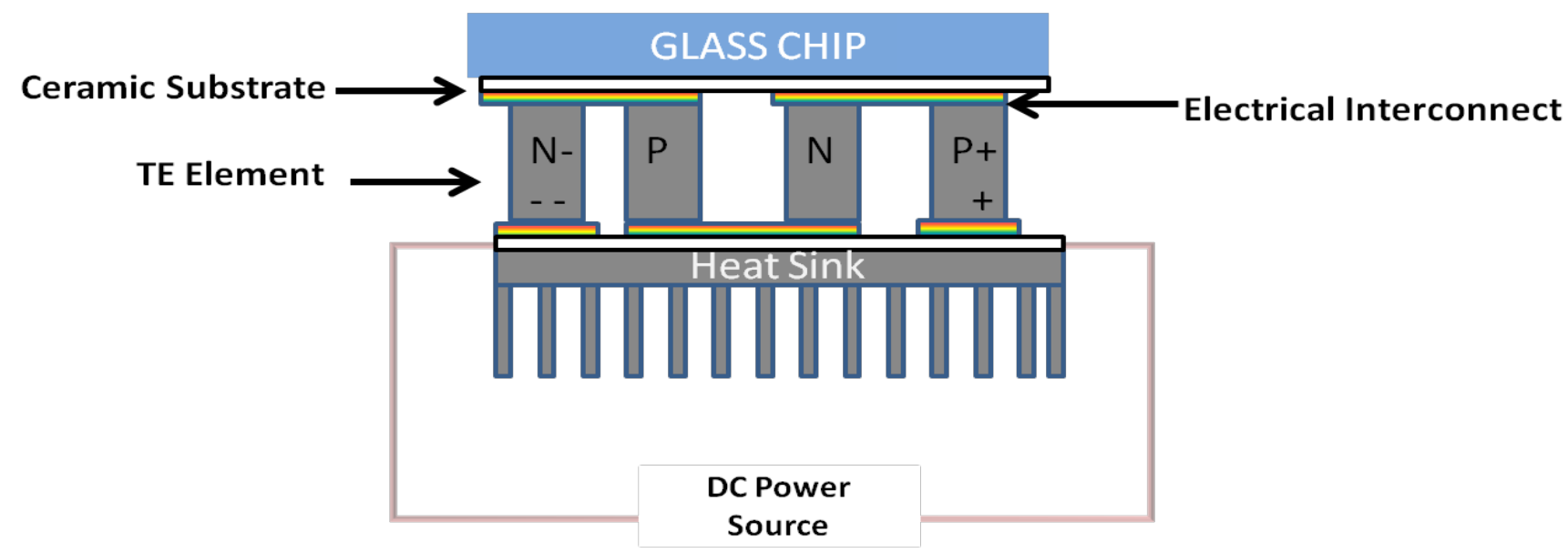

Figure 2-6. A diagram of a TEM (adapted from Ferrotec website with permission) 


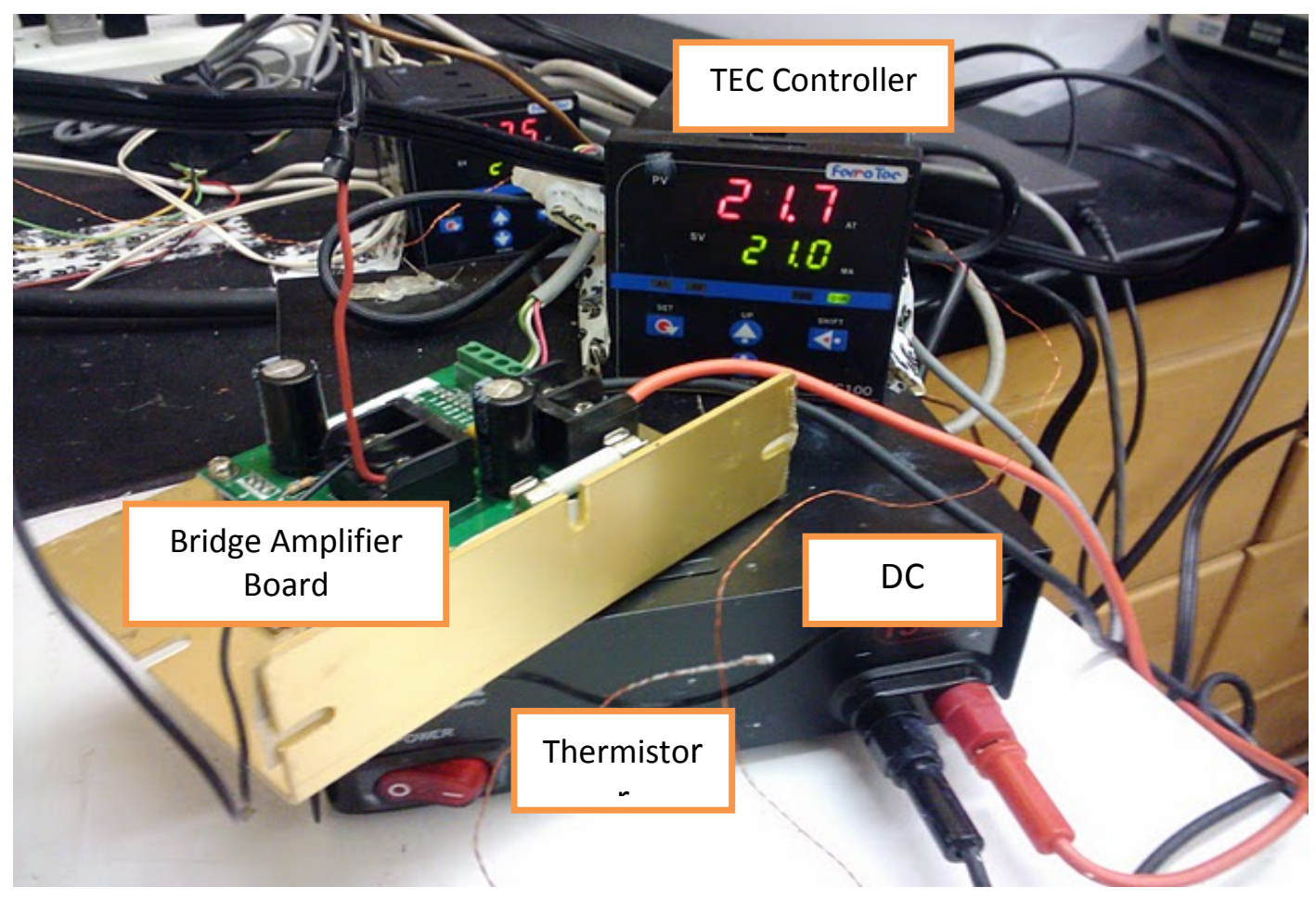

Figure 2-7. A photograph showing the other components of a TEM temperature control system that works with TEM-a thermistor, a bridge amplifier board, a Thermoelectric Cooler (TEC) controller and a DC power supply. 


\subsection{Materials and methods}

3-(N-morpholino)propanesulfonic acid (MOPS) was purchased from Sigma-Aldrich (St. Louis, MO, USA). Methanol was obtained from EMD Biosciences (La Jolla, CA, USA). Fluorescein isothiocyanate (FITC) was purchased from Thermo Fisher Scientific, Inc (Rockford, IL, USA). The lipids DMPC and DHPC were purchased from Avanti Polar Lipids (Alabaster, AL, USA). Deionized water was generated from an Elga Purelab ultra water system (Lowell, MA, USA). The phospholipid preparations were comprised of DMPC and DHPC for which $[\mathrm{DMPC}] /[\mathrm{DHPC}]=2.5$. The preparations were dissolved in $50 \mathrm{mM}$ MOPS, buffered at $\mathrm{pH}$ of 7 , to achieve an aqueous solution that consists of $10 \%$, $15 \%$, or $20 \%$ total phospholipid. The preparation was subjected to three freeze-thaw cycles. The solution was centrifuged for 10 minutes at 10,000 RPM. The UV-visible absorbance detector (Chrom Tech, Inc, Apple Valley, MN, USA) was set at $254 \mathrm{~nm}$. The UV-visible detector was connected to a computer and monitored with IGor Pro 5 software (WaveMetrics Inc, OR, USA) as described previously.

\subsection{Results and discussion}

\subsubsection{Results of viscosity measurement in circular ducts}

The power-law fitting results obtained in circular ducts are summarized numerically in Table 2-1. The calculated apparent viscosity values at each shear rate are listed in Table 2-2 and Table 2-3 and are graphically shown in Figure 2-8. The shear stress and shear rate effectively fit the power-law equation $\left(R^{2}>0.9\right)$ at all temperatures yielding a 
power-law index, $n$, less than 1 . This indicates that the DMPC-DHPC preparations are power-law fluids, the apparent viscosity decreases as shear rate increases (i.e. shearthinning). Table 2-2 depicts the apparent viscosity versus shear rate measured with 100 $\mu \mathrm{m}$ inner diameter (id) and $50 \mu \mathrm{m}$ id capillaries. The power-law index changes at the gel-phase-transition temperature of DMPC, which is $24^{\circ} \mathrm{C}$. For the $50 \mu \mathrm{m}$ id capillary the power-law index is $\sim 0.55$ below $24^{\circ} \mathrm{C}$ and $\sim 0.85$ above $24^{\circ} \mathrm{C}$. For the $100 \mu \mathrm{m}$ id capillary the power-law index is $\sim 0.55$ below $24{ }^{\circ} \mathrm{C}$ and $\sim 0.73$ above $24{ }^{\circ} \mathrm{C}$. Figure $2-8$ graphically displays the apparent viscosities measured with $100 \mu \mathrm{m}$ id and $50 \mu \mathrm{m}$ id capillaries at each shear rate under different temperatures. In Figure 2-8, it is clear that the scattered points fall into two groups, which are the measurement conducted at temperature below and above $24{ }^{\circ} \mathrm{C}$. The apparent viscosity measurements at $29^{\circ} \mathrm{C}$ obtained with the $50 \mu \mathrm{m}$ id capillary were repeated with a phospholipid preparation with $[\mathrm{DMPC}] /[\mathrm{DHPC}]=2.5,15 \%$ phospholipid, 85\% aqueous MOPS. As shown in Table 2-3, measured apparent viscosity was higher for $20 \%$ phospholipid than $15 \%$ phospholipid at each shear rate, and the power-law index was similar, with $n=0.89$ compared to $n=$ 0.84 for $20 \%$ and $15 \%$ phospholipid preparation, respectively.

The consistency index describes the overall viscosity range of the flow curve and, as per Equation 2-10, the apparent viscosity. Both the consistency index and apparent viscosity generally increase with temperature (see Tables 2-1 and Figure 2-8). Measurements of consistency index that are made at temperatures below the gel-phase transition $\left(20\right.$ and $\left.23^{\circ} \mathrm{C}\right)$ are similar for the phospholipid preparation with $[\mathrm{DMPC}] /[\mathrm{DHPC}]=2.5,20 \%$. Above the transition temperature, the consistency index 
and apparent viscosity differ with shear stress. At $26^{\circ} \mathrm{C}$ and $29^{\circ} \mathrm{C}$ lower apparent viscosities were obtained with the smaller inner diameter capillary, which may result from a wall effect. Measurements of the consistency index repeated with a phospholipid preparation at a lower concentration with $15 \%[\mathrm{DMPC}] /[\mathrm{DHPC}]=2.5$, confirming that the $20 \%$ preparation has a larger apparent viscosity $(K=1.7)$ than the $15 \%(K=1.2)$ phospholipid preparation (see Tables 2-3). 


\begin{tabular}{|c|c|c|c|c|c|c|}
\hline $\begin{array}{l}\text { Temperature } \\
\left({ }^{\circ} \mathrm{C}\right)\end{array}$ & $\begin{array}{l}\text { Power Law } \\
\text { Index (n) }\end{array}$ & $\begin{array}{l}\text { Consistency } \\
\text { Index }\left(\mathrm{K}^{\prime},\right. \\
\left.\mathrm{Pa} \cdot \mathrm{s}^{\mathrm{n}}\right) \text {, } \\
\text { uncorrected }\end{array}$ & $\begin{array}{l}\text { Consistency } \\
\text { Index }\left(K_{D h}\right. \\
\left.\text { Pa. } s^{n}\right) \text {, } \\
\text { corrected }\end{array}$ & $\begin{array}{l}\text { Sheer rate range } \\
\left(\sec ^{-1}\right)\end{array}$ & $\mathrm{R}^{2}$ & Count \\
\hline \multicolumn{7}{|c|}{$50 \mu \mathrm{m}$ inner diameter } \\
\hline 20 & $0.52 \pm 0.05$ & $0.3 \pm 0.1$ & $0.26 \pm 0.09$ & $46-930$ & 0.979 & 15 \\
\hline 23 & $0.60 \pm 0.04$ & $0.23 \pm 0.06$ & $0.21 \pm 0.06$ & $50-610$ & 0.989 & 15 \\
\hline 26 & $0.79 \pm 0.09$ & $0.8 \pm 0.2$ & $0.8 \pm 0.2$ & $5.8-27$ & 0.967 & 15 \\
\hline 29 & $0.89 \pm 0.04$ & $1.8 \pm 0.1$ & $1.7 \pm 0.1$ & $1.4-7.5$ & 0.995 & 15 \\
\hline \multicolumn{7}{|c|}{$100 \mu \mathrm{m}$ inner diameter } \\
\hline 20 & $0.57 \pm 0.02$ & $0.22 \pm 0.04$ & $0.20 \pm 0.04$ & $330-3200$ & 0.996 & 15 \\
\hline 23 & $0.53 \pm 0.03$ & $0.27 \pm 0.06$ & $0.24 \pm 0.05$ & $240-3100$ & 0.993 & 18 \\
\hline 26 & $0.76 \pm 0.05$ & $2.5 \pm 0.3$ & $2.4 \pm 0.3$ & $1.7-17$ & 0.988 & 18 \\
\hline 29 & $0.70 \pm 0.04$ & $3.8 \pm 0.3$ & $3.6 \pm 0.3$ & $1.8-12$ & 0.995 & 15 \\
\hline 31 & $0.75 \pm 0.02$ & $3.9 \pm 0.1$ & $3.7 \pm 0.1$ & $1.4-9.6$ & 0.999 & 15 \\
\hline
\end{tabular}

Consistency Index based on Rabinowitsch-Mooney correction for circular ducts, data are fit with nonlinear regression. 


\section{Table 2-2.}

Apparent Viscosity Calculated for 20\% Phospholipid in Circular Ducts

\begin{tabular}{lll}
$26{ }^{\circ} \mathbf{C}, \mathbf{5 0} \boldsymbol{\mu} \mathrm{m}$ circular duct & \\
\hline $\mathrm{n}=0.79 \pm 0.09$ & $\mathrm{~K}=0.8 \pm 0.2$ \\
& Pa. $\mathrm{s}^{\mathrm{n}}$ & $\mathrm{R}^{2}=0.967$ \\
\hline
\end{tabular}

\section{$\mathrm{du} / \mathrm{dr}\left(\mathrm{s}^{-1}\right)$}

range $=6.2-28.2$

28.2

16.3

11.4

8.6

6.2

\section{Appt viscosity (Pa.s)}

$0.4 \pm 0.2$

$0.5 \pm 0.2$

$0.5 \pm 0.2$

$0.5 \pm 0.2$

$0.5 \pm 0.2$

\begin{tabular}{lll}
$26{ }^{\circ} \mathrm{C}, \mathbf{1 0 0} \boldsymbol{\mu m}$ circular duct & \\
\hline $\mathrm{n}=0.73 \pm 0.05$ & $\begin{array}{l}\mathrm{K}=2.5 \pm 0.3 \\
\text { Pa. } \mathrm{s}^{\mathrm{n}}\end{array}$ & $\mathrm{R}^{2}=0.988$ \\
\hline
\end{tabular}

$\mathrm{du} / \mathrm{dr}\left(\mathrm{s}^{-1}\right)$

range $=2.4-18.3$

$\begin{array}{rl}18.3 & 1.0 \pm 0.2 \\ 11.6 & 1.1 \pm 0.2 \\ 7.9 & 1.3 \pm 0.2 \\ 4.3 & 1.6 \pm 0.1 \\ 2.4 & 1.9 \pm 0.1\end{array}$

$29{ }^{\circ} \mathrm{C}, \mathbf{1 0 0} \mu \mathrm{m}$ circular duct

\begin{tabular}{lll}
\hline $\mathrm{n}=0.70 \pm 0.04$ & $\mathrm{~K}=3.8 \pm 0.3$ & $\mathrm{R}^{2}=0.995$ \\
& Pa. $\mathrm{s}^{\mathrm{n}}$ & \\
\hline
\end{tabular}

$\mathrm{du} / \mathrm{dr}\left(\mathrm{s}^{-1}\right)$

range $=2.0-13.3$

13.3

7.9

4.8

3.7

2.0
$1.7 \pm 0.2$

$1.9 \pm 0.2$

$2.2 \pm 0.2$

$2.4 \pm 0.2$

Appt viscosity (Pa.s)

$2.7 \pm 0.3$

Consistency Index based on Rabinowitsch correction for circular ducts, data are fit with nonlinear regression 


\section{Table 2-3.}

Apparent Viscosity for $15 \%$ and $20 \%$ Phospholipid in $50 \mu \mathrm{m}$ Circular Ducts

\section{$29^{\circ} \mathrm{C}, 15 \%$ phospholipid}

\begin{tabular}{lll}
\hline$n=0.84 \pm 0.03$ & $K=1.24 \pm 0.09$ & $R^{2}=0.997$ \\
& $P a . s^{n}$ & \\
\hline
\end{tabular}

$\mathrm{du} / \mathrm{dr}\left(\mathrm{s}^{-1}\right)$

range $=2.3-12.7$

12.7

9.0

6.0

4.1

2.3
Appt viscosity (Pa.s)

$$
\begin{aligned}
& 0.82 \pm 0.09 \\
& 0.87 \pm 0.09 \\
& 0.94 \pm 0.09 \\
& 1.00 \pm 0.09 \\
& 1.11 \pm 0.09
\end{aligned}
$$

$29{ }^{\circ} \mathrm{C}, \mathbf{2 0 \%}$ phospholipid

\begin{tabular}{lll}
\hline $\mathrm{n}=0.89 \pm 0.04$ & $\mathrm{~K}=1.7 \pm 0.1$ & $\mathrm{R}^{2}=0.997$ \\
& $\mathrm{~Pa} . \mathrm{s}^{\mathrm{n}}$ & \\
\hline
\end{tabular}

$$
\mathrm{du} / \mathrm{dr}\left(\mathrm{s}^{-1}\right)
$$

range $=1.5-7.8$

$\begin{array}{ll}7.8 & 1.3 \pm 0.2 \\ 5.4 & 1.4 \pm 0.2 \\ 3.7 & 1.5 \pm 0.2 \\ 2.9 & 1.6 \pm 0.2 \\ 1.5 & 1.7 \pm 0.1\end{array}$

Consistency Index based on Rabinowitsch-Mooney correction for circular ducts, data are fit with nonlinear regression 
Figure 2-8. Plots of apparent viscosity vs. shear rate for $q 2.5,20 \%$ phospholipid in circular ducts

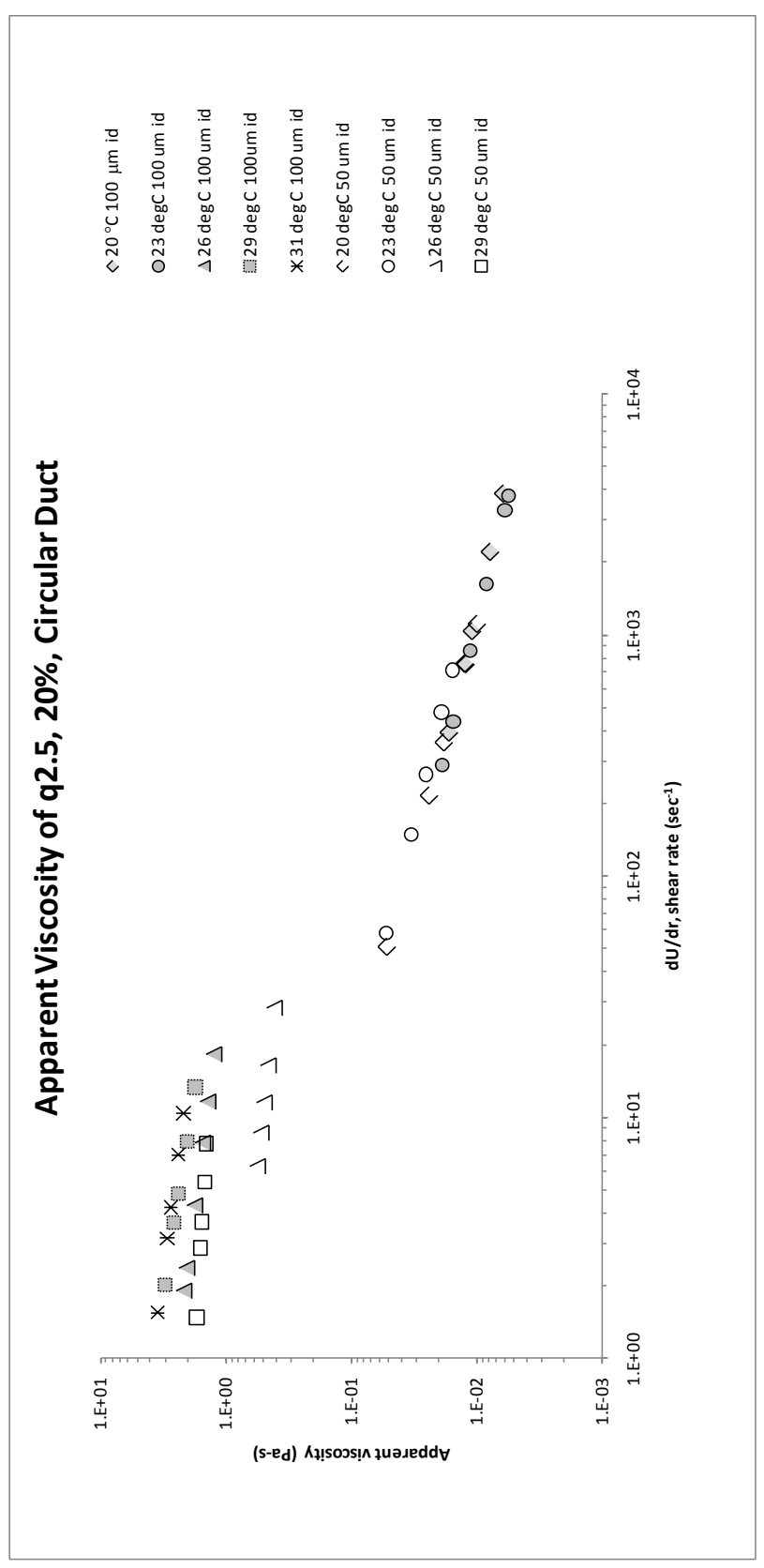




\subsubsection{Problems with bench-top measurement result}

While data shown above have confirmed that phospholipid preparations are shear thinning power-law fluids and that the thermally tunable viscosity is a function of shear rate and the composition of the lipids, several problems were encountered. One problem was poor reproducibility of elution time. For instance, the reproducibility of retention time for phospholipid preparation at $23{ }^{\circ} \mathrm{C}$ ranges from $15 \%$ to $70 \%$ at each pressure point. This may be attributed to the fact that residual phospholipids in the capillary retarded the flow. This problem was alleviated by implementing a flushing protocol in the beginning of the day as well as in between runs. By implementing this flush, the reproducibility of the time in subsequent data sets improved to $\leq 10 \%$ with exception of two points being $15 \%$ with the flushing protocol. Another issue identified was that lipids older than two weeks sometimes were not reproducible. This indicates the preparation has changed probably due to microbacterial growth. Therefore, lipids were prepared and used within two weeks.

Another concern with bench-top results is the observed relationship between flow behavior index, $n$, and temperature. The flow behavior index, $n$, increases with increase in temperature from $20^{\circ} \mathrm{C}$ to $29^{\circ} \mathrm{C}$. For instance, $n$ increased from 0.52 to 0.89 for $20 \%$ phospholipids measured with $50 \mu \mathrm{m}$ id capillary, indicating fluid behavior becomes more

Newtonian at higher temperature. This is in contrast with the observations reported ${ }^{22}$ in which the flow behavior index decreased with increase in temperature. Flaws in the benchtop experimental design are likely responsible for this discrepancy. These issues are related to sample introduction and temperature control. 


\subsubsection{Sources of error due to flaws in the experimental setup}

The method of sample introduction may introduce error in bench-top measurements because of the variability of the injection position. With this custom built setup, injection is accomplished by pushing a fluorescent sample plug into the capillary from the injection reservoir by pressure. The injection time was arbitrarily determined varying from $1 \mathrm{~s}$ to $10 \mathrm{~s}$, therefore the position of the plug following upon injection will vary depending upon the viscosity of the preparation the capillary is filled with. The actual length that the injection plug travelled is not known and varies between runs at different temperatures.

Temperature control with this experimental setup also generates error. This is due to the fact that the part of capillary from the detection window to the end could not be temperature controlled. The total length of the fused silica capillary used was $90 \mathrm{~cm}$ long with $9 \mathrm{~cm}$ from the detection window to the end. The part of the capillary before the detection window was temperature controlled with a water bath. However, the rest of the capillary enters the detection cell, and cannot be thermally controlled. The uncontrolled part of the capillary was located close to the UV lamp in the detector and was possibly warmed up by the heat dissipated from the lamp. In addition, the end of the capillary terminates in a glass vial filled with $2 \mathrm{~mL}$ of deionized water. Since the glass vial was not temperature controlled, the temperature of the water in the vial depends on the ambient room temperature.

The uncontrolled portion of the capillary only constitutes one tenth of the entire capillary, but it significantly impacts the viscosity measurement. In Figure 2-9 A-C an 
analogy of resistors in electric circuit is used to illustrate how the unregulated part of the capillary could affect the measurement, causing the result to appear more nonNewtonian at temperatures lower than room temperature and more Newtonian at temperatures higher than room temperature. As introduced previously, the viscosities of phospholipid preparations undergoes significant increase from $20^{\circ} \mathrm{C}$ to $29^{\circ} \mathrm{C}$. At $24^{\circ} \mathrm{C}$ the viscosity increases sharply. Phospholipid preparations at different temperatures have different flow resistance; therefore, they can be perceived as resistors of different values. Analogous to resistors in series, the total resistance is proportional to the length of the phospholipid-filled capillary. In this illustration the room temperature was assumed to be $24^{\circ} \mathrm{C}$. Two temperatures $20^{\circ} \mathrm{C}$ and $29^{\circ} \mathrm{C}$ were used to demonstrate the effect where the set temperature is lower or higher than room temperature. Figure 2-9B shows that when viscosity is measured at $20^{\circ} \mathrm{C}$, while the room temperature is $24^{\circ} \mathrm{C}$, fluid driven by shear force will encounter a higher flow resistance for the last one tenth of the distance of the capillary. Figure $2-9 \mathrm{C}$ shows that when measured at $29^{\circ} \mathrm{C}$, fluid will experience less resistance at the last one tenth of the distance of the capillary. The resistance is a function of the viscosity of lipids at different temperatures and as well as the length of capillary at that temperature. However, the impact of the resistance is dependent upon the shear rate. At high shear rate, the difference in flow resistance has low but detectable impact on the elution time whereas at low shear rate, the difference in flow resistance has more significant impact. Figure 2-9B illustrates these shear-ratedependent impacts on viscosity measurement at $20^{\circ} \mathrm{C}$ when the room temperature is $24^{\circ} \mathrm{C}$. In this case, at high shear rate, the measured elution time will be close to the true elution time when the entire capillary is maintained at $20^{\circ} \mathrm{C}$. However, at low shear rate, 
the impact of the resistance difference is more pronounced so that the measured elution time is slower than the true elution time. As a result, the measured viscosity will appear much larger than the actual viscosity. When power-law fitting is performed, this will result in a measured $n$ value that is smaller than the true $n$ value. This effect is graphically shown in the plot in Figure 2-9B, where the square represents the measured data and the diamond depict the true value that should be obtained when the whole capillary is thermally regulated. Similarly, Figure 2-9C illustrates the case of measuring at $29^{\circ} \mathrm{C}$ while the room is $24^{\circ} \mathrm{C}$. In this case, the unregulated part of the capillary will cause the $n$ value to be greater than actual. 


\section{Shear force}

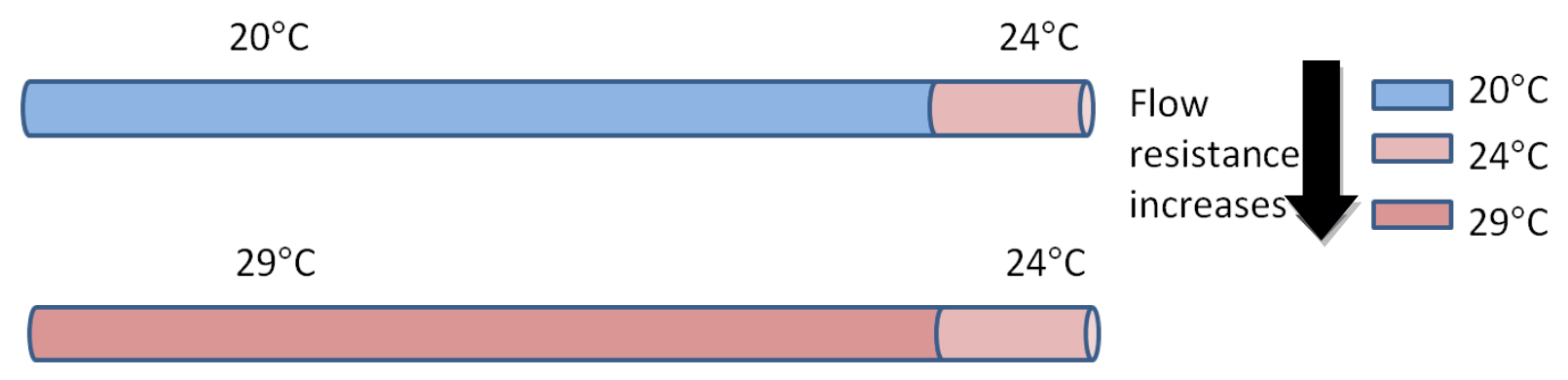

Figure 2-9 Illustrates how errors result from the fact that one tenth of the capillary was not temperature controlled. The diagrams in A show the example condition that the room temperature was $24^{\circ} \mathrm{C}$ and two temperatures $20^{\circ} \mathrm{C}$ and $29^{\circ} \mathrm{C}$ were used to demonstrate the effect where the set temperature is lower or higher than room temperature. 


\section{$20^{\circ} \mathrm{C}$}

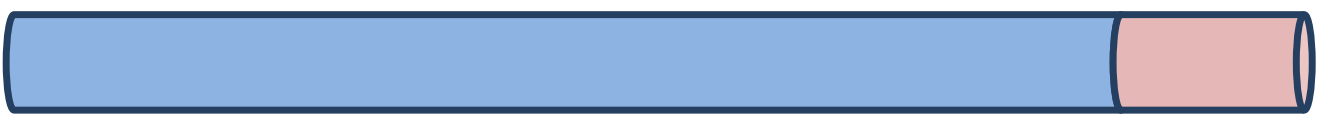

At higher shear rate, time measured $\approx$ time actual, $\eta$ measured $\approx \eta$ actual

At lower shear rate, time measured $>$ time actual, $\eta$ measured $>\eta$ actual

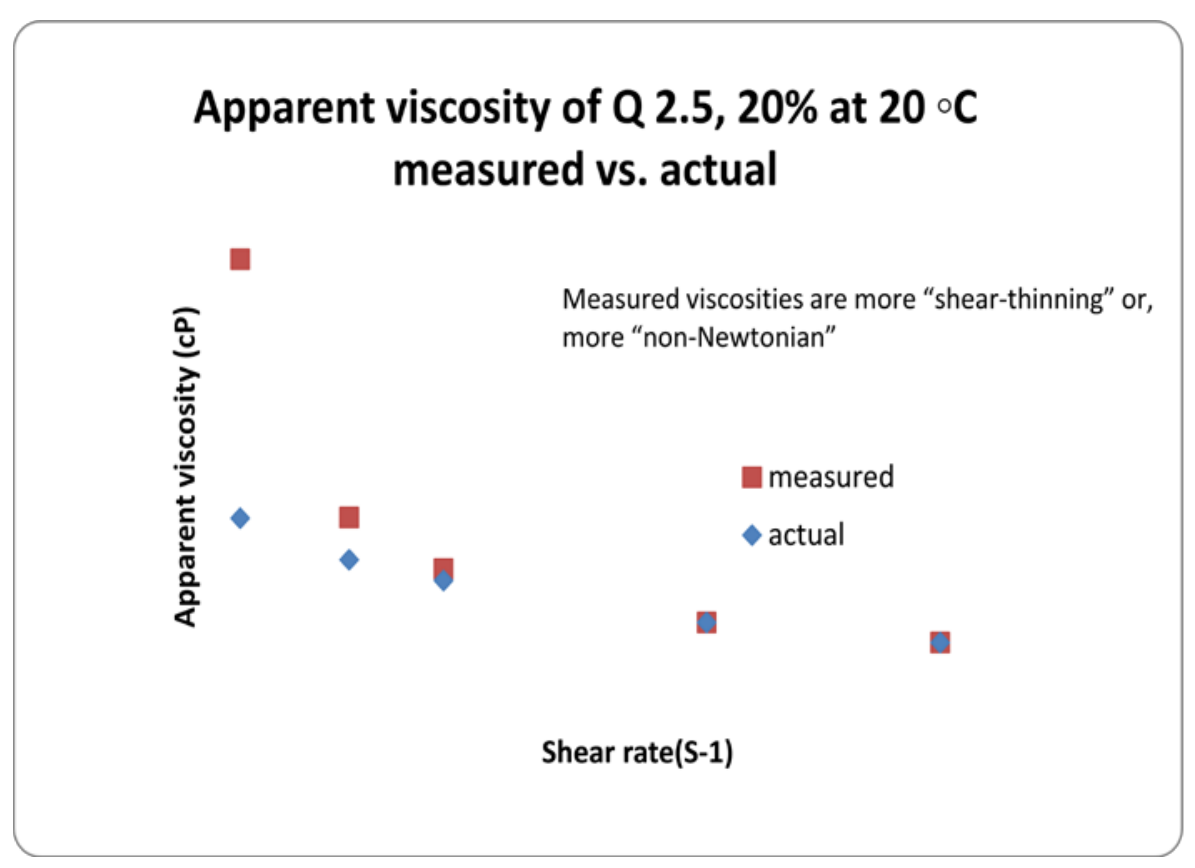

Figure 2-9B Illustrates that when the viscosity measurement of a shear thinning power law fluid is carried out at a temperature lower than room temperature, the $n$ value obtained is smaller than the actual value. 


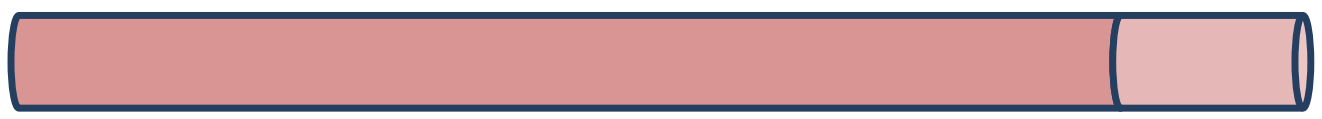

At higher shear rate, time measured $\approx$ time actual, $\eta$ measured $\approx \eta$ actual

At lower shear rate, $t$ measured $<\mathrm{t}$ actual, $\eta$ measured $<\eta$ actual

\section{Apparent Viscosity at $29^{\circ} \mathrm{C}$ measured vs. actual}

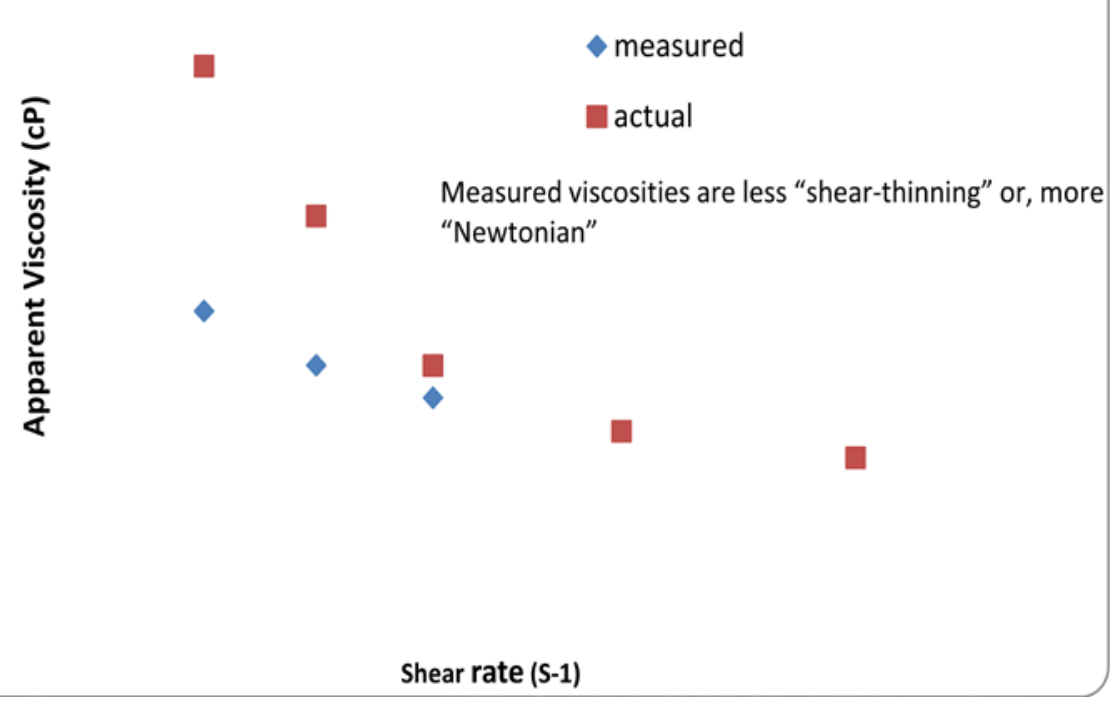

Figure 2-9C illustrates that when the viscosity measurement of a shear thinning power law fluid is carried out at a temperature greater than room temperature, the $n$ value obtained is larger than the actual value. 
Transferring the viscosity study onto a microfluidic chip can overcome thermostatting issues and injection artifacts of the bench-top setup. For temperature control, the whole 2" by 4" glass chip can be controlled with two TEMs. Because the lamp is located far away from the microscope stage, heat from the lamp is not a concern. For sample introduction, with the double T design and connecting ports that can hold about $30 \mu \mathrm{L}$ liquid, electrokinetic injection of a small plug is readily achievable. Meanwhile onchip viscosity characterization offers additional benefits. The pattern is designed with a turn in the channel such that the detection point can be chosen between $3.2 \mathrm{~cm}$ or 10.6 $\mathrm{cm}$ without moving the stage, which provides the opportunity to study the effect of turns in the microfluidic channels. Moreover, since the goal is to integrate the media into microfluidic applications, it is important to study the fluid behavior under the same conditions as microfluidic chips. Circular ducts on bench-top measurements are not representative of trapezoidal microfluidic channels. Therefore, it is desirable to transfer the measurement to microchip. 


\section{References}

1. Archer-Hartmann, S. A.; Crihfield, C. L.; Holland, L. A., Microscale Exoglycosidase Processing of Trastuzumab Using Capillary Electrophoresis. Electrophoresis 2011, 32, 3491-3498.

2. Archer-Hartmann, S. A.; Sargent, L. M.; Lowry, D. T.; Holland, L. A., Microscale Exoglycosidase Processing and Lectin Capture of Glycans with Phospholipid Assisted Capillary Electrophoresis Separations. Anal. Chem. 2011, 83 (7), 27402047;

3. Luo, R.; Archer-Hartmann, S. A.; Holland, L. A., Transformable Capillary Electrophoresis for Oligosaccharides Separation Using Phospholipid Additives. Anal. Chem. 2010, 82, 1228-1233.

4. Nieh, M. P.; Raghunathan, V. A.; Glinka, C. J.; Harroun, T. A.; Pabst, G.; Katsaras, J., Magnetically alignable phase of phospholipid "bicelle" mixtures is a chiral nematic made up of wormlike micelles. Langmuir 2004, 20, 7893-7;

5. Harroun, T. A.; Koslowsky, M.; Nieh, M.-P.; de_Lannoy, C.-F.; Raghunathan, V. A.; Katsaras, J., Comprehensive examination of mesophases formed by DMPC and DHPC mixtures. Langmuir 2005, 21, 5356-61.

6. Walker, L. M., Rheology and structure of worm-like micelles. Current Opinion in Colloid and Interface Science 2001, 6 (5-6), 451-456.

7. Triba, M. N.; Warschawski, D. E.; Devaux, P. F., Reinvestigation by phosphorus NMR of lipid distribution in bicelles. Biophys. J. 2005, 88, 1887-1901;

8. Rowe, B. A.; Neal, S. L., Fluorescence probe study of bicelle structure as a function of temperature: developing a practical bicelle structure model. Langmuir 2003, 19, 2039-48;

9. Luchette, P. A.; Vetman, T. N.; Prosser, R. S.; Hancock, R. E. W.; Nieh, M.-P.; Glinka, C. J.; Krueger, S.; Ka tsaras, J., Morphology of fast-tumbling bicelles: a small angle neutron scattering and NMR study. Biochim. Biophys. Acta 2001, 1513, 83-94.

10. Mills, J. O.; Holland, L. A., Membrane mediated capillary electrophoresis: Interaction of cationic peptides with bicelles. Electrophoresis 2004, 25, 1237-42;

11. Hwang, J. S.; Oweimreen, G. A., Anomalous viscosity behavior of a bicelle system with various molar ratios of short- and long-chain phospholipids. The Arabian Journal for Science and Engineering, 2003, 28 (1A), 43-49. 
12 .Pappas, T.; Holland, L., Fluid Steering in a Microfluidic Chip by Means of Thermally Responsive Phospholipids. Sens. Actuators, B, Chem 2008, 128, 427-434.

13. Vold, R. R.; Prosser, R. S., Magnetically oriented phospholipid bilayered micelles for structural studies of polypeptides: does the ideal bicelle exist? J. Magn. Reson., Ser B 1996, 113, 267-71.

14. Mohanty, P.; Lee, J.; Glover, K.; Landskron, K., Discoid Bicelles as Efficient Templates for Pillared Lamellar Periodic Mesoporous Silicas at $\mathrm{pH} 7$ and Ultrafast Reaction Times. Nanoscale Research Letters 2011, 6 (1), 1-5.

15. Landers, J. P., Handbook of capillary and microchip electrophoresis and associated microtechniques. 2007.

16. Luo, R.; Archer-Hartmann, S. A.; Holland, L. A., Transformable capillary electrophoresis for oligosaccharide separations using phospholipid additives. Anal. Chem., 2010; Vol. 82.

17. Tian, H.; Landers, J., Hydroxyethylcellulose as an effective polymer network for DNA analysis in uncoated glass microchips: optimization and application to mutation detection via heteroduplex analysis. Analytical Biochemistry, 2002; Vol. 309, pp 212223.

18. Heller, C., Finding a universal low-viscosity polymer for DNA separation. 988; Vol. 19, pp 1691-1698.

19. de Waele, A., Viscometry and Plastometry. Journal of the Oil \& Colour Chemists Association, 1923; Vol. 6, pp 33-69.

20. Macosko, C. W., Rheology principles, measurements, and applications. VCH Publishers.Inc: New York, NY, 1994.

21. Chaturani, P.; Narasimman, S., Flow of power-law fluids in cone-plate viscometer. Acta.Mechanica, 1990; Vol. 82, pp 197-211.

22. Flynn, A.; Ducey, M.; Yethiraj, A.; Morrow, M. R., Dynamic properties of bicellar lipid mixtures observed by rheometry and quadrupole echo decay. Langmuir, 2011; Vol. 28, pp 2307-3062. 


\section{CHAPTER 3}

Viscosity Measurements of DMPC-DHPC

Phospholipid Preparations in Trapezoidal

Microfluidic Channel 


\subsection{Introduction}

As discussed in Chapter Two, there are several benefits to transferring phospholipid viscosity characterization to microfluidic channels. For one thing, the microfluidic measurement was designed to overcome the bench-top instrumental flaws in temperature control. For another, when the viscosity measurement is performed in microfluidic channels, the results reflect the fluid behavior under conditions similar to those of microfluidic applications. Moreover, viscosity measurements in microfluidic chips are able to provide more meaningful information than bench-top instrument with circular capillaries. For example, the isotropic etching process used to fabricate the microfluidic channels in a glass chip gives rise to a trapezoidal cross sectional area. This is different from the circular cross sectional area of the capillary used in capillary viscometry in the bench-top measurement. In order to understand the effect of channel geometry on the fluid behavior of phospholipids, viscosity characterization of phospholipids in chip is required. Also, non-Newtonian fluid behavior can be affected by turns in the microfluidic channel. The in-chip measurement was performed in a microfluidic channel with a turn which enabled the study of turn effect on the rheology of phospholipids.

Several microfluidic devices have been developed to elucidate the non-linear rheology. These have included glass devices fabricated by chemical wet-etching ${ }^{1,2}$ as well as devices cast with PDMS. ${ }^{3-6}$ In this study, glass chips were used because they are ideal for the capillary electrophoresis as well as other applications on-chip. Viscosity characterization in glass channels has been based on gradient dilution, ${ }^{1}$ differential 
wicking across a serpentine channel and open chamber ${ }^{2}$ and measurement of channel penetration based on capillary action. ${ }^{3-5}$ Rheological characterization in these devices has provided a means to study biopolymers, ${ }^{1}$ organic materials, ${ }^{5}$ and polymers. ${ }^{2}$ In this study, as with benchtop measurement, viscosity characterization in chip was based on the principle of capillary viscometry, therefore it represented the conditions for microfluidic applications.

This chapter focuses on the viscosity characterization of phospholipid preparations in microfluidic channels as a function of temperature and pressure. The instrument setup used for in-chip measurement has complete temperature control as detailed in the materials and methods. The data are in good agreement with the expected trend and were compared with the bench-top measurement results. Measurements were made at two detection points—before and after the turn in the microfluidic channel to investigate the turn effect.

\subsection{Materials and methods}

\subsubsection{Reagents and materials}

Materials and reagents used are the same as detailed in Chapter Two. They are listed below for readers' convenience. 3-(N-morpholino)propanesulfonic acid (MOPS) was purchased from Sigma-Aldrich (St. Louis, MO, USA). Methanol was obtained from EMD Biosciences (La Jolla, CA, USA). Biotium (Hayward, CA, USA) was the source for 1aminopyrene-3, 6, 8-trisulfonic acid (APTS). Fluorescein isothiocyanate (FITC) was 
purchased from Thermo Fisher Scientific, Inc (Rockford, IL, USA). The lipids DMPC and DHPC were purchased from Avanti Polar Lipids (Alabaster, AL, USA). Deionized water was generated from an Elga Purelab ultra water system (Lowell, MA, USA). The phospholipid preparations were comprised of DMPC and DHPC for which [DMPC]/ $[D H P C]=2.5$. The preparations were dissolved in $50 \mathrm{mM}$ MOPS, buffered at $\mathrm{pH}$ of 7 to achieve an aqueous solution that consists of $10 \%, 15 \%$, or $20 \%$ total phospholipid. The preparation was subjected to three freeze-thaw cycles. The solution was centrifuged for 10 minutes at 10,000 RPM.

\subsubsection{Glass chip fabrication}

Microfluidic glass chips are fabricated using standard photolithography and chemical etching with hydrofluoric acid. The patterning and etching steps of the glass chip fabrication are outlined in Figure 3-1. Borosilicate substrates deposited with chromium and photoresist layers are purchased from Telic Company $(2 \times 4$ inch Valencia, CA, USA). Masks were custom-designed using FreeHand (Adobe, San Jose, CA, USA) and printed on the film by International Phototool Company (Colorado Springs, CO). At the first step, the glass chip with the mask aligned on top is exposed to the UV light. In the following developing step, photoresist of the area where it was exposed to the UV light was removed. Consequently, in those regions the chromium layer was removed in the next step of chromium etching. Following chromium etching, a mixture of hydrofluoric acid and nitric acid was used to etch the glass surface where the chromium layer was removed. The solution etches the channel in all directions, creating a cross-sectional area that can be approximated as a trapezoid. The depth of the etched channel can be 


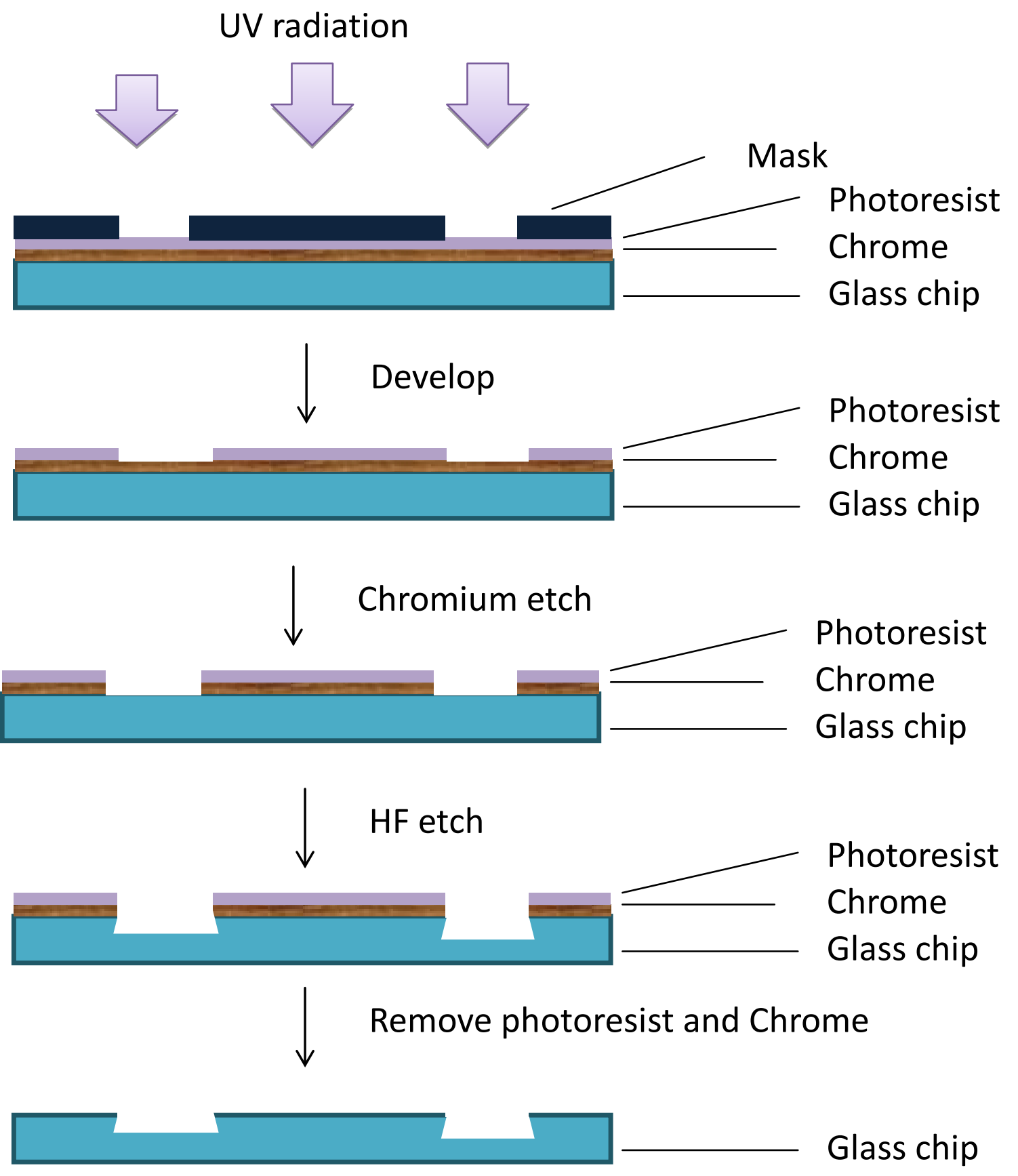

Figure 3-1. Cross sectional view showing the glass chip fabrication process-UV photo patterning followed by wet chemical etching 
controlled via the etching time. After the glass channel is etched, extra photoresist and chromium was removed with acetone and chromium etch respectively. Etched channels are examined with an Alpha-Step IQ surface profiler (KLA Tencor, CA, USA). Micro drill bit of diameter $360 \mu \mathrm{m}$ was used to drill holes at each end of the micro channel for connecting to the real world. After soaking in piranha wash, the glass chip with etched channel is bound to a blank glass chip of the same size in a high temperature oven.

\subsubsection{Measurement of apparent viscosity in non-circular ducts}

Viscosity measurements were performed using a microfluidic chip visualized with a Nikon Eclipse TE300 inverted microscope (Vermont Optechs, Inc., Charlotte, VT, USA) as shown in Figure 3-2. A cross sectional view of the chip on the microscope stage is shown in Figure 3-3. The design for the chip used for viscosity measurement is shown in Figure 3-4. The cross-sectional geometry of the channel can be roughly approximated as a trapezoid $38.9 \mu \mathrm{m}$ high with dimensions of the upper channel, lower channel, and sidewall of $163.3 \mu \mathrm{m}, 105.1 \mu \mathrm{m}$ and $48.6 \mu \mathrm{m}$ respectively. Bonded port connectors (C360-400, LabSmith, Livermore, CA, US) were connected to each reservoir on the chip with epoxy. The chip was mounted on a thermally regulated stage equipped with a Hamamatsu C10600 ORCA-R² digital camera (Hamamatsu Photonics, K.K., Hamamatsu City, Japan). The temperature of the microscope stage was controlled by two thermoelectric modules (TEM) (Ferrotec, NH, USA), each attached to a heat sink and high flow computer fan. The temperature of the pressure reservoir is also controlled with a TEM. The PEEK tubing connecting the pressure reservoir and the glass chip was temperature regulated with a circulating water bath as shown in Figure 3-2. 
Figure 3-2. A Instrumental setup for measurement of viscosity in chip

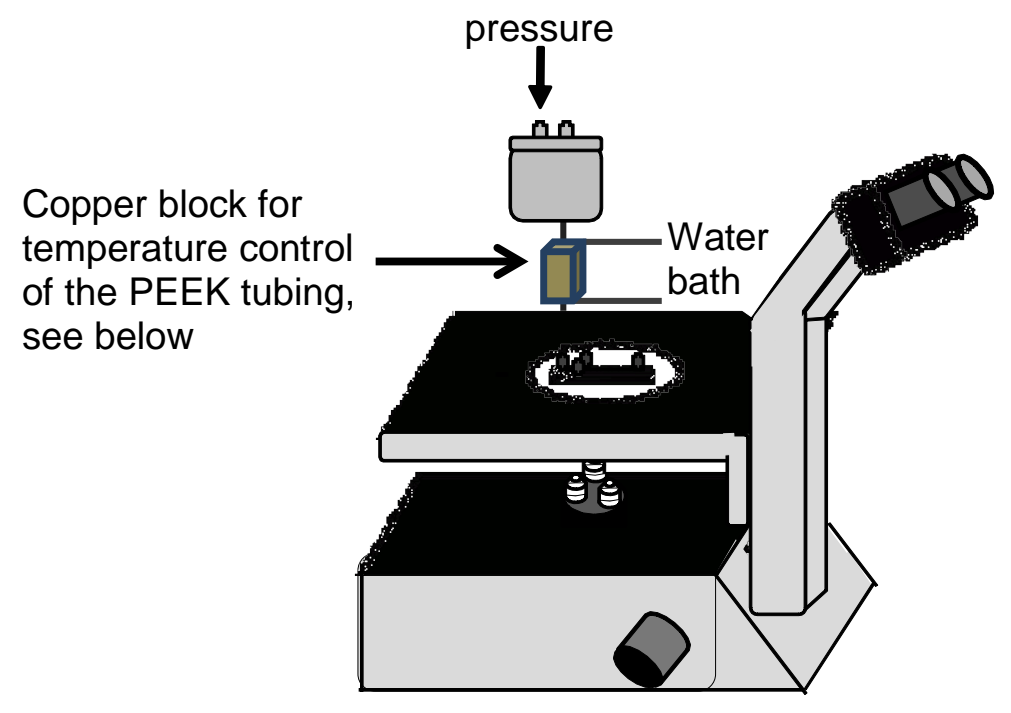

Figure 3-2 B. Top view of the copper block for temperature control of PEEK tubing PEEK tubing $\longrightarrow-\begin{aligned} & \text { Copper tubing } \\ & \text { connecting to the } \\ & \text { water bath }\end{aligned}$ 
Photographs of the temperature controlled setup for in-chip viscosity measurement are as shown in Figures 3-5 and 3-6. In order to flush the microfluidic chip, PEEK tubing (Upchurch Scientific, WA, USA) was used to connect port 1 to a stainless steel pressure reservoir. All flushes were performed by first flowing solutions from port 1 to port 2 as well as port 3 , and then blocking ports 2 and 3 to force fluid flow to port 4 . Each morning the chip was flushed with $3 \mathrm{~mL}$ water, $0.5 \mathrm{~mL}$ MOPS, and then filled with phospholipid.

For viscosity measurements performed in the microfluidic chip, fluorescent sample 1aminopyrene-3, 6, 8-trisulfonic acid (APTS) doped phospholipids were loaded into the separation channel electrokinetically so that transport to the detection window was due solely to the pressure-driven fluid flow initiated at the beginning of the run. To accomplish this, the channel was loaded with phospholipid. Ports 1, 3, and 4 were filled with $30 \mu \mathrm{L}$ of MOPS, while port 2 was filled with $30 \mu \mathrm{L}$ of APTS dissolved in $50 \mathrm{mM}$ MOPS buffered to $\mathrm{pH}$ 7. The APTS was electrokinetically injected from port 2 to 3 for 30 seconds at $-1.0 \mathrm{kV}$ with a high voltage power supply (CZE1000R, Spellman High Voltage Electronics, NY, USA). The solution in ports 2 and 3 was removed and the ports blocked. The APTS plug was used to visualize the velocity. The APTS plug was then driven through the channel to port 4 from the pressure driven flow applied at port 1 . The APTS plug elution time was determined at a specific temperature. The time for the fluorescent plug to arrive at the detection window reflected the fluid velocity. The apparent viscosity was measured before the channel turn, at a position $3.2 \mathrm{~cm}$ from the beginning of the channel. Apparent viscosity was also measured after the turn at a channel length of $10.6 \mathrm{~cm}$ (see Figure 3-4). 


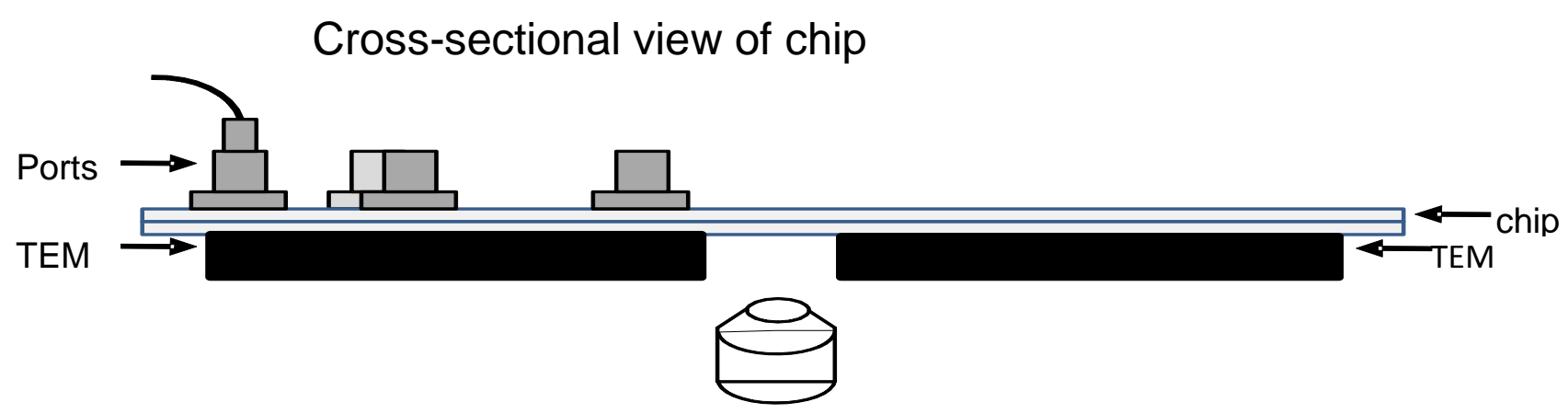

Figure 3-3. Schematic of the setup and the temperature control for viscosity 


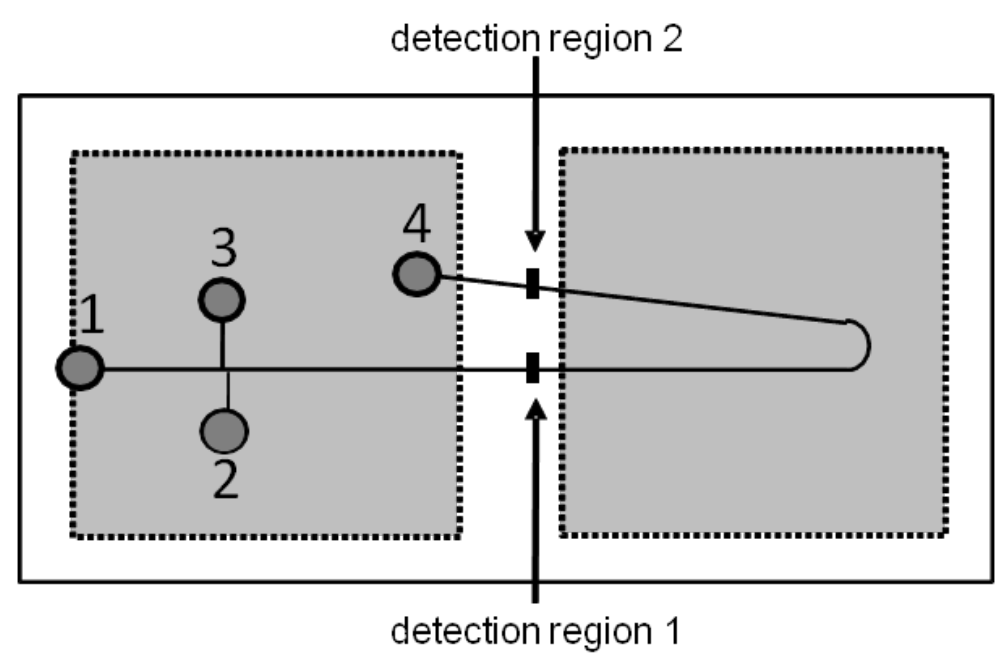

Figure 3-4. Top view of the chip design used for in-chip viscosity measurement. Detection region 1 is $3.2 \mathrm{~cm}$ from the injection cross while the detection region 2 after turn is $10.6 \mathrm{~cm}$ from the injection cross. 


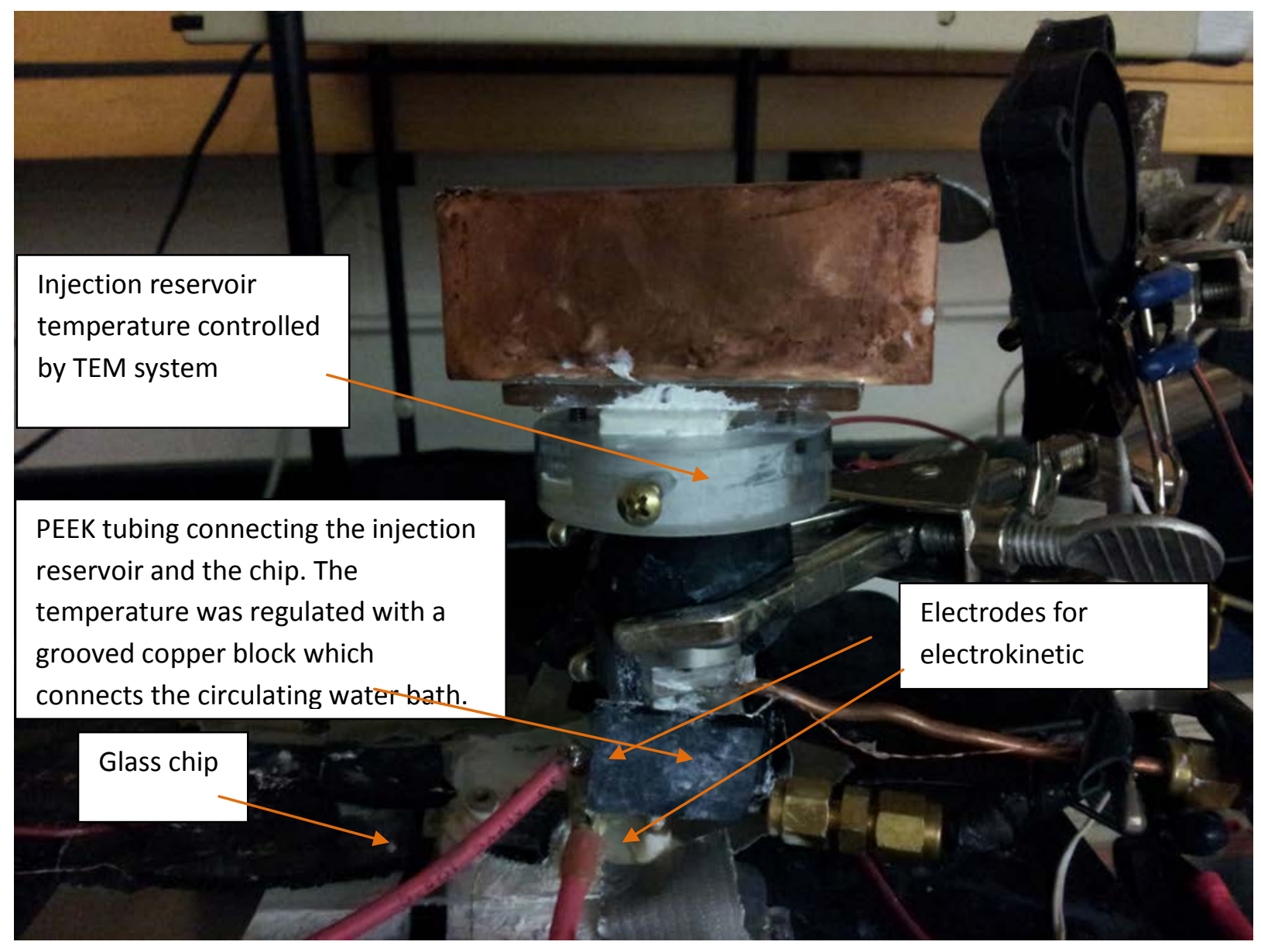

Figure 3-5. A photograph showing the setup for viscosity measurement in microfluidic chip 


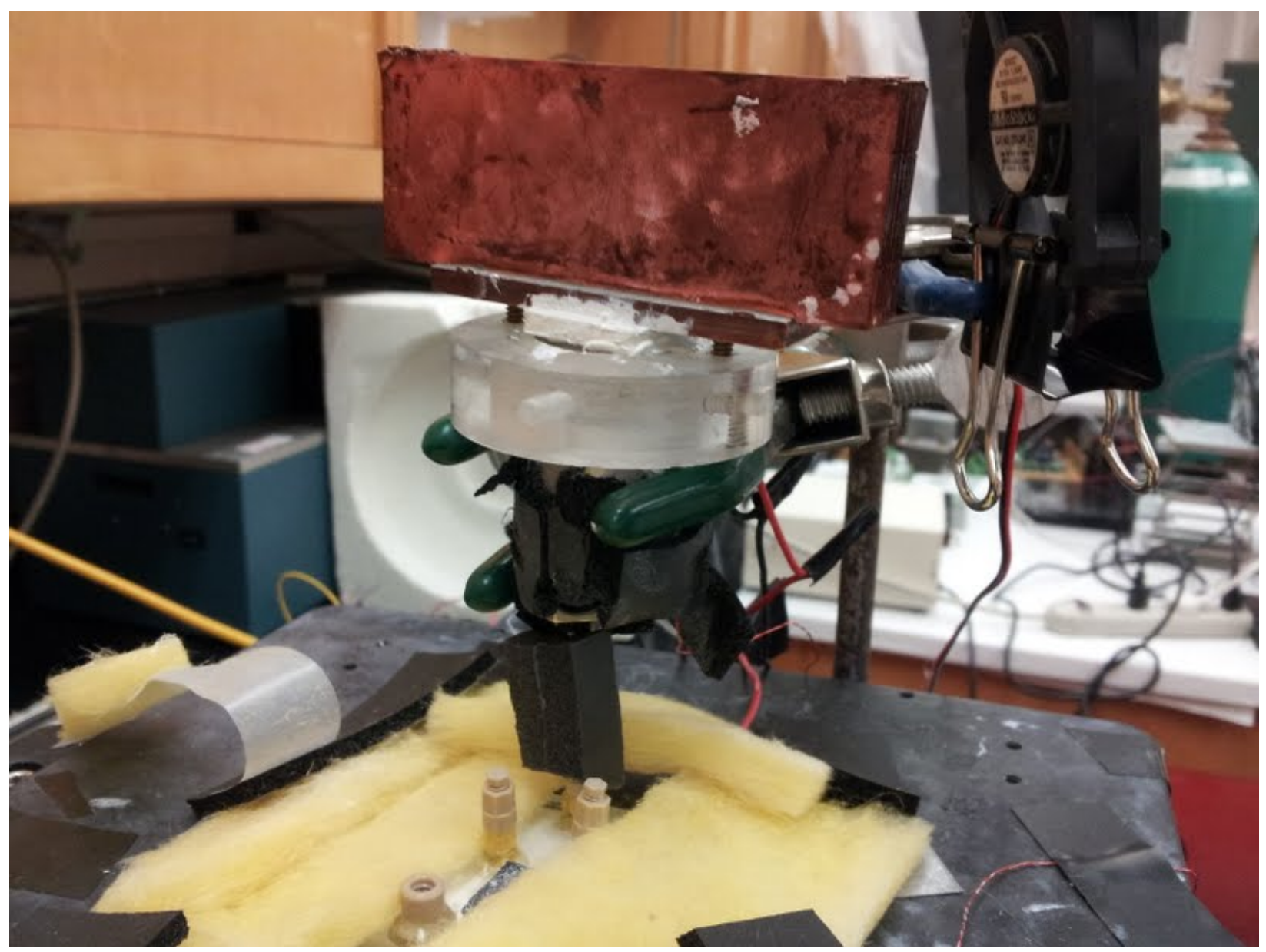

Figure 3-6. A photograph showing the temperature regulation of the pressure reservoir and the PEEK tubing connecting the pressure reservoir to the glass chip. The pressure reservoir was temperature controlled by a TEM directly on the top of the lid of the reservoir. A heat sink was held in contact with the TEM to dissipate heat. A computer fan was blowing towards the heat sink as shown. A Plexiglas ring was used to hold the TEM and the copper heat sink in place. The chip design is as shown in Figure 3-4. 


\subsection{Results and discussion}

\subsubsection{An example of data processing}

Below is an example of data processing. Hydraulic diameter of the trapezoidal cross section was calculated and the cross sectional area of the channel was treated as a circle which has a diameter of the calculated hydraulic diameter. Data were processed in the same manner as detailed in Chapter Two.

Hydraulic diameter is calculated as $D_{h}=4($ Area $) /($ Perimeter) from the chip dimensions, which for this data set are listed below. Given these measurements, the cross sectional area is $5.22 \mathrm{E}-09 \mathrm{~m}^{2}$, the perimeter is $0.000366 \mathrm{~m}$, and the hydraulic diameter is $0.0000572 \mathrm{~m}$.

\begin{tabular}{|lr|}
\hline Length $(\mathrm{m})$ & 0.0320 \\
top $(\mathrm{m})$ & 0.000163 \\
bottom $(\mathrm{m})$ & 0.000105 \\
height $(\mathrm{m})$ & 0.00151 \\
sidewall $(\mathrm{m})$ & 48.6 \\
\hline
\end{tabular}

Table 3-1A. Dimensions of the microchip 
Measurements of elution time are made at a known temperature and applied pressure.

Data for $23^{\circ} \mathrm{C}, \mathrm{L}=3.2 \mathrm{~cm}$ are listed in the table below.

\begin{tabular}{cc}
\hline $\begin{array}{c}\text { pressure } \\
\mathbf{( P a})\end{array}$ & $\begin{array}{c}\text { ave } \\
\text { time } \\
(\mathbf{s e c})\end{array}$ \\
$4.1 \mathrm{E}+05$ & $2.7 \mathrm{E}+00$ \\
$3.4 \mathrm{E}+05$ & $4.2 \mathrm{E}+00$ \\
$2.8 \mathrm{E}+05$ & $7.0 \mathrm{E}+00$ \\
$2.1 \mathrm{E}+05$ & $1.3 \mathrm{E}+01$ \\
$1.4 \mathrm{E}+05$ & $2.3 \mathrm{E}+01$ \\
$6.9 \mathrm{E}+04$ & $7.2 \mathrm{E}+01$ \\
$3.4 \mathrm{E}+04$ & $2.7 \mathrm{E}+02$ \\
$1.4 \mathrm{E}+04$ & $6.2 \mathrm{E}+02$ \\
\hline
\end{tabular}

Table 3-1B. Elution time at each applied pressure 
The shear stress, $\tau$, is calculated from hydraulic diameter, pressure and channel length as $\tau=D_{h} \Delta P / 4 L$, while the shear rate, $\gamma$, is estimated from linear velocity (i.e. channel length divided by elution time) and hydraulic diameter as $\gamma^{\prime}=8 \mathrm{~V} / \mathrm{D}_{\mathrm{h}}$ as follows:

\begin{tabular}{cccc}
\hline $\begin{array}{c}\text { pressure } \\
(\mathrm{Pa})\end{array}$ & $\begin{array}{c}\mathrm{D}_{\mathrm{h}} \mathrm{PI4L} \\
(\mathrm{Pa})\end{array}$ & $\begin{array}{c}\text { ave } \\
\text { time } \\
(\mathbf{s e c})\end{array}$ & $\begin{array}{c}\gamma^{\prime} \\
\left(\mathrm{sec}^{-1}\right)\end{array}$ \\
$4.1 \mathrm{E}+05$ & $1.8 \mathrm{E}+02$ & $2.7 \mathrm{E}+00$ & $1.7 \mathrm{E}+03$ \\
$3.4 \mathrm{E}+05$ & $1.5 \mathrm{E}+02$ & $4.2 \mathrm{E}+00$ & $1.1 \mathrm{E}+03$ \\
$2.8 \mathrm{E}+05$ & $1.2 \mathrm{E}+02$ & $7.0 \mathrm{E}+00$ & $6.4 \mathrm{E}+02$ \\
& & & \\
$2.1 \mathrm{E}+05$ & $9.2 \mathrm{E}+01$ & $1.3 \mathrm{E}+01$ & $3.4 \mathrm{E}+02$ \\
& & & \\
$1.4 \mathrm{E}+05$ & $6.2 \mathrm{E}+01$ & $2.3 \mathrm{E}+01$ & $1.9 \mathrm{E}+02$ \\
& & & \\
$6.9 \mathrm{E}+04$ & $3.1 \mathrm{E}+01$ & $7.2 \mathrm{E}+01$ & $6.3 \mathrm{E}+01$ \\
& & & \\
$3.4 \mathrm{E}+04$ & $1.5 \mathrm{E}+01$ & $2.7 \mathrm{E}+02$ & $1.6 \mathrm{E}+01$ \\
& & & \\
$1.4 \mathrm{E}+04$ & $6.2 \mathrm{E}+00$ & $6.2 \mathrm{E}+02$ & $7.2 \mathrm{E}+00$ \\
& & & \\
\hline
\end{tabular}

Table 3-1C. Calculated estimated shear rate at each shear stress 
The shear stress and shear rate are fit to the equation $\tau=K^{\prime}\left(\gamma^{\prime}\right)^{n^{\prime}}$ using nonlinear regression. For this data set, the fit was obtained using Graphpad software yielding the following values:

$\mathrm{K}^{\prime}=4.4_{8} \pm 0.7_{7} \mathrm{~Pa} \cdot \mathrm{s}^{\mathrm{n}}, \mathrm{n}^{\prime}=0.50_{6} \pm 0.02_{5}, \mathrm{R}^{2}=0.994$

The uncorrected power-law index ( $\left.n^{\prime}\right)$ and uncorrected consistency index ( $\left.K^{\prime}\right)$ are determined. Because the data fit the power-law equation $n^{\prime}=n$. The power-law index is then used to adjust K' using the Rabinowitsch-Mooney (R-M) correction. To accomplish this shear rate is now estimated as $Y=(3 / 4+1 / 4 n) 8 V / D_{h}$ as outlined below.

\begin{tabular}{|c|c|c|c|c|}
\hline $\begin{array}{l}\text { pressure } \\
\text { (Pa) }\end{array}$ & $\begin{array}{c}\mathrm{D}_{\mathrm{h}} \mathrm{P} / 4 \mathrm{~L} \\
(\mathrm{~Pa})\end{array}$ & $\begin{array}{l}\text { ave } \\
\text { time } \\
(\mathrm{sec})\end{array}$ & $\begin{array}{l}8 \mathrm{~V} / \mathrm{D}_{\mathrm{h}} \\
\left(\mathrm{sec}^{-1}\right)\end{array}$ & $\begin{array}{l}\text { Shear } \\
\text { rate } \\
\left(\sec ^{-1}\right)\end{array}$ \\
\hline $4.1 \mathrm{E}+05$ & $1.8 \mathrm{E}+02$ & 2.7E+00 & $1.7 \mathrm{E}+03$ & $2.1 \mathrm{E}+03$ \\
\hline $3.4 \mathrm{E}+05$ & $1.5 \mathrm{E}+02$ & $4.2 \mathrm{E}+00$ & $1.1 \mathrm{E}+03$ & $1.3 \mathrm{E}+03$ \\
\hline $2.8 \mathrm{E}+05$ & $1.2 \mathrm{E}+02$ & $7.0 \mathrm{E}+00$ & $6.4 \mathrm{E}+02$ & $8.0 \mathrm{E}+02$ \\
\hline $2.1 E+05$ & $9.2 E+01$ & 1.3E+01 & $3.4 \mathrm{E}+02$ & $4.2 \mathrm{E}+02$ \\
\hline $1.4 \mathrm{E}+05$ & $6.2 E+01$ & 2.3E+01 & $1.9 \mathrm{E}+02$ & $2.4 \mathrm{E}+02$ \\
\hline $6.9 \mathrm{E}+04$ & $3.1 E+01$ & $7.2 \mathrm{E}+01$ & $6.3 \mathrm{E}+01$ & $7.8 \mathrm{E}+01$ \\
\hline $3.4 E+04$ & $1.5 E+01$ & 2.7E+02 & 1. $6 \mathrm{E}+01$ & $2.0 \mathrm{E}+01$ \\
\hline $1.4 \mathrm{E}+04$ & $6.2 \mathrm{E}+00$ & $6.2 \mathrm{E}+02$ & $7.2 \mathrm{E}+00$ & $8.9 \mathrm{E}+00$ \\
\hline
\end{tabular}

Table 3-1D. Calculated shear rate at each shear stress 
The shear stress and this estimate of shear rate are fit to the equation $\tau=K\left({ }^{\mathrm{du}} / \mathrm{dr}\right)^{\mathrm{n}}$ using nonlinear regression. For this data set the fit obtained using Graphpad software, yielding the following: $K=4.0 \pm 0.7 P a \cdot s^{n}, n=0.51 \pm 0.03, R^{2}=0.994$ Once $\mathrm{K}$ and $\mathrm{n}$ are obtained the apparent viscosity is calculated as $\eta_{A}=K\left({ }^{d u} / d r\right){ }^{n-1}$

\begin{tabular}{|c|c|c|c|c|c|}
\hline $\begin{array}{l}\text { pressure } \\
\text { (Pa) }\end{array}$ & $\begin{array}{c}\mathrm{D}_{\mathrm{h}} \mathrm{P} / 4 \mathrm{~L} \\
(\mathrm{~Pa})\end{array}$ & $\begin{array}{c}\text { ave } \\
\text { time } \\
\text { (sec) }\end{array}$ & $\begin{array}{l}8 \mathrm{~V} / \mathrm{D}_{\mathrm{h}} \\
\left(\mathrm{sec}^{-1}\right)\end{array}$ & $\begin{array}{l}\text { Shear } \\
\text { rate } \\
\left(\sec ^{-1}\right)\end{array}$ & $\begin{array}{c}\text { Apparent } \\
\text { viscosity(Pa.s) }\end{array}$ \\
\hline $4.1 E+05$ & $1.8 \mathrm{E}+02$ & $2.7 E+00$ & 1.7E+03 & $2.1 E+03$ & 0.09 \\
\hline $3.4 \mathrm{E}+05$ & $1.5 E+02$ & $4.2 E+00$ & $1.1 E+03$ & $1.3 E+03$ & 0.12 \\
\hline $2.8 E+05$ & $1.2 \mathrm{E}+02$ & $7.0 E+00$ & $6.4 \mathrm{E}+02$ & $8.0 E+02$ & 0.15 \\
\hline $2.1 E+05$ & $9.2 E+01$ & $1.3 E+01$ & $3.4 \mathrm{E}+02$ & $4.2 E+02$ & 0.20 \\
\hline $1.4 \mathrm{E}+05$ & $6.2 E+01$ & $2.3 E+01$ & $1.9 E+02$ & $2.4 E+02$ & 0.27 \\
\hline $6.9 \mathrm{E}+04$ & $3.1 E+01$ & $7.2 \mathrm{E}+01$ & $6.3 \mathrm{E}+01$ & $7.8 \mathrm{E}+01$ & 0.5 \\
\hline $3.4 E+04$ & $1.5 E+01$ & $2.7 E+02$ & $1.6 \mathrm{E}+01$ & $2.0 \mathrm{E}+01$ & 0.9 \\
\hline $1.4 \mathrm{E}+04$ & $6.2 \mathrm{E}+00$ & $6.2 \mathrm{E}+02$ & $7.2 \mathrm{E}+00$ & $8.9 E+00$ & 1.4 \\
\hline
\end{tabular}

Table 3-1E. Calculated apparent viscosity at each shear rate 


\subsubsection{Non-Newtonian flow in trapezoidal cross section channel}

In 1972, Miller ${ }^{7}$ devised a convenient way to estimate the shear stress of a nonNewtonian fluid in ducts of noncircular cross section based on hydraulic diameter $D_{h}$,

$D_{h}=4 A / P$

where $A$ is cross sectional area and $P$ is perimeter, and a geometry factor, $\lambda$ :

$\lambda=\mathrm{fR}$

where $f$ is Fanning friction factor, and $R_{\mathrm{e}}$ is Reynolds number.

In 2006 Bharami et al developed a solution based on $R_{e} \sqrt{A}$, which depends on the perimeter, area and aspect ratio of the cross section to calculate the value of trapezoidal cross section microchannle. ${ }^{8}$ Other scaling factors include the grouping of $\mathrm{P}$ and $\sqrt{A}$ which was devised by Muzychka et al. ${ }^{9}$ Once shear stress and shear rate of the flow in the microchannel of trapezoidal cross section are calculated, the power-law fitting can be performed by plotting shear stress against shear rate as described above.

Different methods of fitting were compared and it was found that all fitting results agree to the result calculated as shown in 3.3 .1 within $10 \%$. Therefore that method was employed. In Table 3-1, four columns show the fitting result of $\mathrm{K}$ obtained using four methods. They are: 1.) Treat the trapezoidal channel as the circular duct with the diameter equal to the hydraulic diameter $\mathrm{D}_{\mathrm{h}}$ of the trapezoid, and then us R-M correction as the example shown in 3.3.1. 2.) Use Bharami's model for trapezoidal shape. ${ }^{8} 3$ and 4.) Model the trapezoid as a rectangular of two extreme cases which 
have the same height as the trapezoid and the top(the longer) or the bottom (the shorter) of the trapezoid as the base respectively and using Muzychka's method. ${ }^{9}$ These methods yield the same value of $n(0.26 \pm 0.02)$ and as for $\mathrm{K}$, there is no significant difference between method 1 and 2 as listed in Table 3-2. The fact that method 3 yielded the same result as method 1 and 2, whereas the result of method 4 deviated indicates that the longer base of the trapezoid has greater influence than the shorter base on the geometrical effect. Therefore, data obtained in chip were processed using method 1. 
Table 3-2. Fitting results of $\mathrm{K}$ using different methods

\begin{tabular}{ccccc}
\hline & $\begin{array}{c}\text { 1. } \mathbf{R}-\mathrm{M} \\
\text { correction, } \\
\text { circular }\end{array}$ & $\begin{array}{c}\text { 2.Trapedoidal } \\
\text { Correction }\end{array}$ & $\begin{array}{c}\text { 3.Rectangular } \\
\mathbf{1}\end{array}$ & $\begin{array}{c}\text { 4.Rectangular } \\
\mathbf{2}\end{array}$ \\
\hline $\mathrm{K}\left(\mathrm{Pa} \cdot \mathrm{s}^{\mathrm{n}}\right)$ & $16_{3} \pm 1.1$ & $15_{5} \pm 1.1$ & $16_{0} \pm 1.1$ & $14.9 \pm 1.1$ \\
\hline
\end{tabular}

Four methods (1-4) are detailed in 3.3.2. DMPC-DHPC of $q=2.5,20 \%$, at $29^{\circ} \mathrm{C}$ after turn chip data were used for this calculation. 
3.3.3 Results of viscosity measurement of phospholipids in microfluidic channel

The data processing shown above was used to treat all the measurement at $23^{\circ} \mathrm{C}, 24$ ${ }^{\circ} \mathrm{C}, 26^{\circ} \mathrm{C}$ and $29^{\circ} \mathrm{C}$ measured before the turn (channel length $3.2 \mathrm{~cm}$ ) and after the turn $(10.6 \mathrm{~cm})$. The apparent viscosity at each shear rate as well as $\mathrm{K}$ and $n$ value are listed in Table 3-3. As shown in the table, all flow behavior indices are less than one which confirms again that phospholipid preparations are a shear-thinning fluid. The apparent viscosity measured before the turn at each temperature was plotted against the corresponding shear rate in Figure 3-7. The characteristic of shear-thinning flow behavior of phospholipids is embodied clearly in the plot that the apparent viscosity decrease as shear rate increases at all temperatures. Also at each shear rate, the apparent viscosity of phospholipids increase with temperature in the temperature range studied $\left(23^{\circ} \mathrm{C}-29^{\circ} \mathrm{C}\right)$. Furthermore, the slope obtained for $23^{\circ} \mathrm{C}$ and $24{ }^{\circ} \mathrm{C}$ is different from that of $26^{\circ} \mathrm{C}$ and $29^{\circ} \mathrm{C}$. The apparent viscosity values at $26^{\circ} \mathrm{C}$ and $29^{\circ} \mathrm{C}$ are higher than at $23^{\circ} \mathrm{C}$ and $24^{\circ} \mathrm{C}$. The trace of $26^{\circ} \mathrm{C}$ and $29^{\circ} \mathrm{C}$ have similar slopes while $23^{\circ} \mathrm{C}$ and $24^{\circ} \mathrm{C}$ have a different set of similar slopes.

Table 3-4 compares the results of viscosity measurement between bench-top instrument and in-chip. As discussed in detail in Chapter Two, there were errors, including the apparent faulty relationship between flow behavior index $n$ and temperature, caused by the flaws in the bench-top instrumental setup. Data obtained in micofluidic chip with temperature fully controlled support this conclusion—as listed in Table 3-3, the in-chip measurements (both before turn and after turn) had a decrease in 
flow index $n$ as temperature increases from $23^{\circ} \mathrm{C}$ to $29^{\circ} \mathrm{C}$, indicating fluid becomes more shear-thinning as temperature increases.

Comparing the in-chip measurement results obtained before turn and after turn, as shown in Table 3-4, there is no significant difference in $n$ value at each temperature. This indicates that the turn in microfluidic channel of such design does not change the flow behavior significantly. However, the $\mathrm{K}$ value generally drops after the turn (it drops by $85 \%$ at $23{ }^{\circ} \mathrm{C}, 81 \%$ at $24{ }^{\circ} \mathrm{C}, 63 \%$ at $26^{\circ} \mathrm{C}$ and $71 \%$ at $29^{\circ} \mathrm{C}$ ). This corresponds to a decrease in apparent viscosity after turn. These data suggest that the turns in the microfluidic channel decrease the apparent viscosity of non-Newtonian fluids such as phospholipids. Continued studies on the effect of turns on non-Newtonian fluids in microfluidic chips will provide necessary information to better utilize this complex fluid for microfluidic applications. 
Table 3-3. Apparent Viscosity Calculated for Each Shear Rate for Trapezoidal Ducts (q $2.5,20 \%)$

\begin{tabular}{|c|c|c|c|}
\hline \multicolumn{4}{|l|}{$23^{\circ} \mathrm{C}$} \\
\hline \multicolumn{2}{|c|}{ Before Turn, $L=3.2 \mathrm{~cm}$} & \multicolumn{2}{|c|}{ After Turn, $\mathrm{L}=10.6 \mathrm{~cm}$} \\
\hline \multicolumn{2}{|c|}{$\mathrm{n}=0.51 \pm 0.03, K=4.0 \pm 0.7 \mathrm{~Pa} \cdot \mathrm{s}^{\mathrm{n}}, \mathrm{R}^{2}=0.9938$} & \multicolumn{2}{|c|}{$n=0.61 \pm 0.03, K=0.6 \pm 0.1 \mathrm{~Pa} \cdot \mathrm{s}^{n}, \mathrm{R}^{2}=0.9937$} \\
\hline $\begin{array}{l}\text { Shear rate }\left(\mathbf{s}^{-1}\right) \\
\text { range }=9-2100\end{array}$ & Appt viscosity (Pa.s) & $\begin{array}{l}\text { Shear rate }\left(\mathbf{s}^{-1}\right) \\
\text { range }=30-2000\end{array}$ & Appt viscosity (Pa.s) \\
\hline $2100 \pm 400$ & $0.09 \pm 0.03$ & $2000 \pm 100$ & $0.03 \pm 0.01$ \\
\hline $1300 \pm 200$ & $0.12 \pm 0.03$ & $1400 \pm 100$ & $0.03 \pm 0.01$ \\
\hline $800 \pm 20$ & $0.15 \pm 0.04$ & $920 \pm 60$ & $0.04 \pm 0.01$ \\
\hline $420 \pm 70$ & $0.20 \pm 0.05$ & $550 \pm 30$ & $0.05 \pm 0.01$ \\
\hline $240 \pm 20$ & $0.27 \pm 0.06$ & $290 \pm 20$ & $0.06 \pm 0.02$ \\
\hline $78 \pm 6$ & $0.5 \pm 0.1$ & $120 \pm 20$ & $0.09 \pm 0.02$ \\
\hline $20 \pm 6$ & $0.9 \pm 02$ & $51 \pm 6$ & $0.12 \pm 0.03$ \\
\hline $9 \pm 4$ & $1.4 \pm 0.4$ & $30 \pm 2$ & $0.15 \pm 0.04$ \\
\hline \multicolumn{4}{|l|}{$24^{\circ} \mathrm{C}$} \\
\hline \multicolumn{2}{|c|}{ Before Turn, $\mathrm{L}=3.2 \mathrm{~cm}$} & \multicolumn{2}{|c|}{ After Turn, L = $10.6050 \mathrm{~cm}$} \\
\hline \multicolumn{2}{|c|}{$\mathrm{n}=0.59 \pm 0.02, K=3.7 \pm 0.5 \mathrm{~Pa} \cdot \mathrm{s}^{\mathrm{n}}, \mathrm{R}^{2}=0.9967$} & \multicolumn{2}{|c|}{$\mathrm{n}=0.65 \pm 0.04, K=0.7 \pm 0.2 \mathrm{~Pa} \cdot \mathrm{s}^{\mathrm{n}}, \mathrm{R}^{2}=0.9901$} \\
\hline $\begin{array}{l}\text { Shear rate }\left(\mathbf{s}^{-1}\right) \\
\text { range }=8-800\end{array}$ & Appt viscosity (Pa.s) & $\begin{array}{l}\text { Shear rate }\left(\mathrm{s}^{-1}\right) \\
\text { range }=6.4-165\end{array}$ & Appt viscosity (Pa.s) \\
\hline $800 \pm 200$ & $0.24 \pm 0.06$ & $930 \pm 70$ & $0.06 \pm 0.02$ \\
\hline $570 \pm 90$ & $0.27 \pm 0.06$ & $630 \pm 30$ & $0.07 \pm 0.03$ \\
\hline $390 \pm 80$ & $0.32 \pm 0.07$ & $440 \pm 40$ & $0.08 \pm 0.03$ \\
\hline $250 \pm 40$ & $0.38 \pm 0.08$ & $270 \pm 20$ & $0.10 \pm 0.03$ \\
\hline $110 \pm 30$ & $0.5 \pm 0.1$ & $150 \pm 20$ & $0.12 \pm 0.04$ \\
\hline $24 \pm 2$ & $1.0 \pm 0.2$ & $72 \pm 5$ & $0.15 \pm 0.05$ \\
\hline $12 \pm 1$ & $1.3 \pm 0.2$ & $38 \pm 7$ & $0.19 \pm 0.06$ \\
\hline $8 \pm 2$ & $1.6 \pm 0.3$ & $16 \pm 7$ & $0.26 \pm 0.08$ \\
\hline
\end{tabular}

Consistency Index based on Rabinowitsch-Mooney correction for circular ducts, data is fit with nonlinear regression 


\begin{tabular}{|c|c|c|c|}
\hline \multicolumn{4}{|l|}{$26{ }^{\circ} \mathrm{C}$} \\
\hline \multicolumn{2}{|c|}{ Before Turn, $L=3.2 \mathrm{~cm}$} & \multicolumn{2}{|c|}{ After Turn, $L=10.6050 \mathrm{~cm}$} \\
\hline \multicolumn{2}{|c|}{$\mathrm{n}=0.29 \pm 0.03, \mathrm{~K}=40 \pm 6 \mathrm{~Pa} \cdot \mathrm{s}^{\mathrm{n}}, \mathrm{R}^{2}=0.9683$} & \multicolumn{2}{|c|}{$\mathrm{n}=0.24 \pm 0.02, K=15 \pm 1 \mathrm{~Pa} \cdot \mathrm{s}^{\mathrm{n}}, \mathrm{R}^{2}=0.9866$} \\
\hline $\begin{array}{l}\text { Shear rate }\left(s^{-1}\right) \\
\text { range }=0.52-220\end{array}$ & Appt viscosity (Pa.s) & $\begin{array}{l}\text { Shear rate }\left(\mathrm{s}^{-1}\right) \\
\text { range }=4.4-260\end{array}$ & Appt viscosity (Pa.s) \\
\hline $220 \pm 20$ & $0.9 \pm 0.2$ & $260 \pm 20$ & $0.22 \pm 0.03$ \\
\hline $110 \pm 20$ & $1.4 \pm 0.3$ & $112 \pm 8$ & $0.41 \pm 0.05$ \\
\hline $37 \pm 5$ & $3.0 \pm 0.6$ & $40 \pm 3$ & $0.9 \pm 0.1$ \\
\hline $11 \pm 1$ & $7 \pm 1$ & $10 \pm 2$ & $2.5 \pm 0.4$ \\
\hline $3.6 \pm 0.3$ & $16 \pm 2$ & $4.4 \pm 0.6$ & $4.9 \pm 0.7$ \\
\hline $1.4 \pm 0.1$ & $31 \pm 5$ & & \\
\hline $0.52 \pm 0.04$ & $60 \pm 10$ & & \\
\hline \multicolumn{4}{|l|}{$29^{\circ} \mathrm{C}$} \\
\hline \multicolumn{2}{|c|}{ Before Turn, $L=3.2 \mathrm{~cm}$} & \multicolumn{2}{|c|}{ After Turn, $L=10.6050 \mathrm{~cm}$} \\
\hline \multicolumn{2}{|c|}{$\mathrm{n}=0.29 \pm 0.04, K=56 \pm 8 \mathrm{~Pa} \cdot \mathrm{s}^{\mathrm{n}}, \mathrm{R}^{2}=0.936$} & \multicolumn{2}{|c|}{$n=0.26 \pm 0.02, K=16 \pm 1 \mathrm{~Pa} \cdot \mathrm{s}^{\mathrm{n}}, \mathrm{R}^{2}=0.9903$} \\
\hline $\begin{array}{l}\text { Shear rate }\left(\mathrm{s}^{-1}\right) \\
\text { range }=0.98-75\end{array}$ & Appt viscosity (Pa.s) & $\begin{array}{l}\text { Shear rate }\left(\mathrm{s}^{-1}\right) \\
\text { range }=2.6-120\end{array}$ & Appt viscosity (Pa.s) \\
\hline $75 \pm 8$ & $2.6 \pm 0.6$ & $120 \pm 20$ & $0.45 \pm 0.07$ \\
\hline $30 \pm 4$ & $5 \pm 1$ & $57 \pm 8$ & $0.8 \pm 0.1$ \\
\hline $11 \pm 2$ & $10 \pm 2$ & $21 \pm 1$ & $1.7 \pm 0.2$ \\
\hline $3.2 \pm 0.3$ & $25 \pm 4$ & $7.2 \pm 0.7$ & $3.8 \pm 0.4$ \\
\hline $1.7 \pm 0.1$ & $39 \pm 6$ & $2.6 \pm 0.2$ & $8.1 \pm 0.7$ \\
\hline $0.98 \pm 0.08$ & $57 \pm 9$ & & \\
\hline
\end{tabular}

Consistency Index based on Rabinowitsch-Mooney correction for circular ducts, data is fit with nonlinear regression 


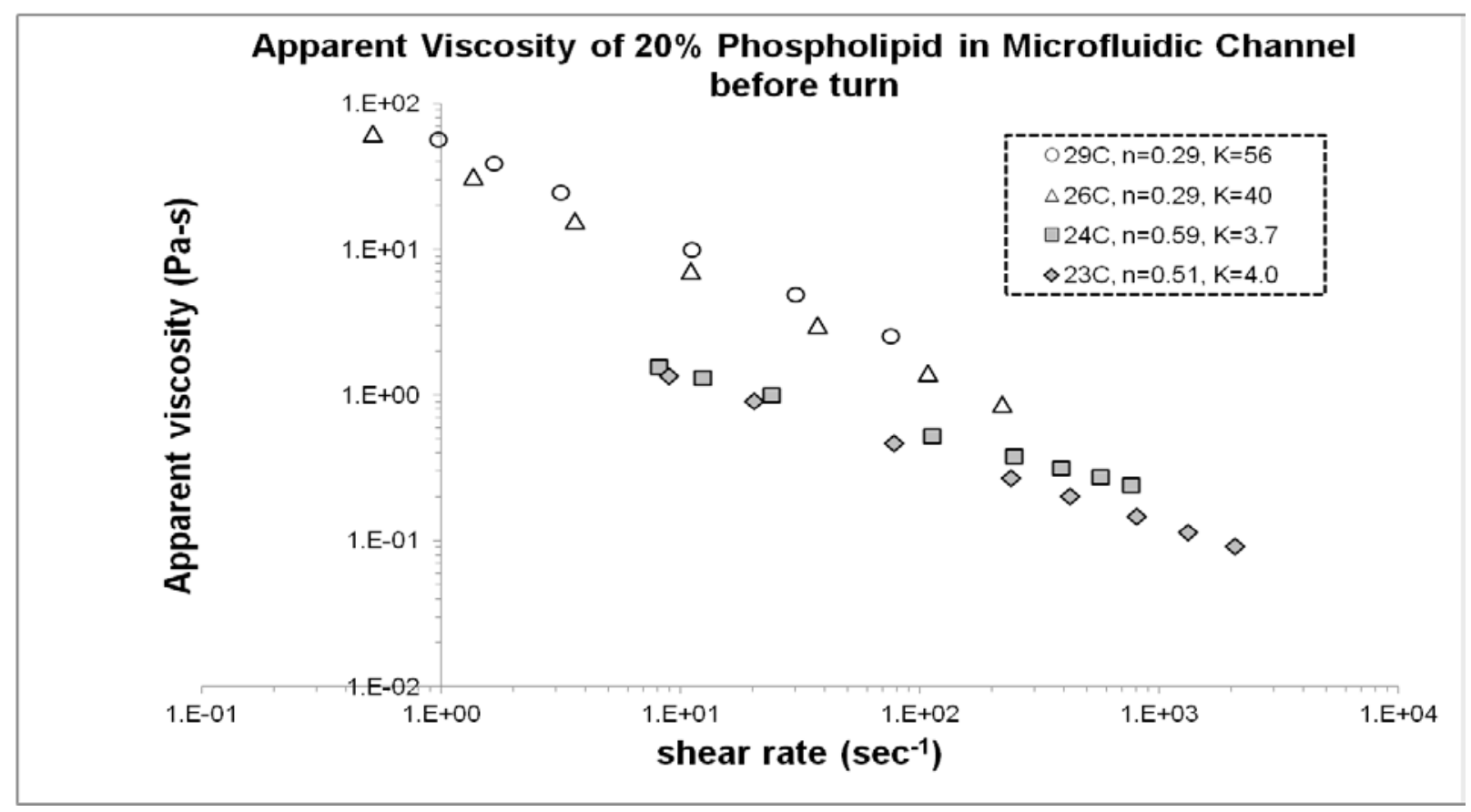

Figure 3-7. A plot of apparent viscosity of a $q=2.5,20 \%$ preparation with change in the shear rate in microfluidic chip (before turn). The values and error associated with each data point are summarized in Table 3-3. 
Table 3-4. Comparison of $\mathrm{K}$ and $n$ values from viscosity measurement of DMPC-DHPC preparations $(q=2.5,20 \%)$ between bench-top instrument and microfluidic chip ( before turn and after turn) at three temperatures.

\begin{tabular}{|c|c|c|c|c|}
\hline Tem & & $\begin{array}{l}50 \mu \mathrm{m} \text { circular } \\
\text { capillary }\end{array}$ & \multirow{2}{*}{$\begin{array}{l}\text { Microchannel } \\
\text { Before Turn } \\
\text { (L=3.2 cm) } \\
4.0 \pm 0.7\end{array}$} & \multirow{2}{*}{$\begin{array}{l}\text { Microchannel } \\
\text { After Turn } \\
\text { (L=10.6 cm) } \\
0.6 \pm 0.1\end{array}$} \\
\hline $23^{\circ} \mathrm{C}$ & $\mathrm{K}$ & $0.3 \pm 0.1$ & & \\
\hline & $\mathrm{n}$ & $0.60 \pm 0.04$ & $0.51 \pm 0.03$ & $0.61 \pm 0.03$ \\
\hline & $\mathrm{R}^{2}$ & 0.979 & 0.9938 & 0.9937 \\
\hline \multirow[t]{3}{*}{$26^{\circ} \mathrm{C}$} & $\mathrm{K}$ & $0.8 \pm 0.2$ & $40 \pm 6$ & $15 \pm 1$ \\
\hline & $\mathrm{n}$ & $0.79 \pm 0.09$ & $0.29 \pm 0.03$ & $0.24 \pm 0.02$ \\
\hline & $\mathrm{R}^{2}$ & 0.967 & 0.9683 & 0.9866 \\
\hline \multirow[t]{3}{*}{$29^{\circ} \mathrm{C}$} & $\mathrm{K}$ & $1.8 \pm 0.1$ & $56 \pm 8$ & $16 \pm 1$ \\
\hline & $n$ & $0.89 \pm 0.04$ & $0.29 \pm 0.04$ & $0.26 \pm 0.02$ \\
\hline & $\mathrm{R}^{2}$ & 0.995 & 0.936 & 0.9903 \\
\hline
\end{tabular}




\subsection{Conclusions}

The viscosity of phospholipid preparations has been characterized in microfluidic channel. The temperature dependent, shear-thinning behavior has been further confirmed. With temperature completely controlled by TEM and water bath, the measured results show that flow index $n$ decreases as temperature increases from below to above the gel transition temperature with a significant increase of apparent viscosity. This may be attributed to the hypothesized elongation of the discoidal assemblies into ribbon or wormlike micelles. Similar to the entanglement of linear polymers, entanglement of wormlike micelles increases the apparent viscosity of the medium.

These findings provide insight into the material performance in microfluidic channels. The thermally tunable viscosity can be utilized to develop applications of the material to steer fluid in microfluidic channels and to enhance capillary electrophoresis separations. The study in Chapter Three was carried out with strict temperature regulation. A water bath was used to control the temperature of the PEEK connecting tubing. 


\section{References}

1. J, L.; A., T., Intrinsic viscosity of polymers and biopolymers measured by microchip. Anal Chem, 2005; Vol. 77, pp 7137-47.

2. Srivastava, N.; Burns, M. A., Analysis of Non-Newtonian liquids using a microfluidic capillary viscometer. Anal. Chem, 2006; Vol. 78, pp 1690-1696.

3. Viola, I.; Pisignano, D.; Cingolani, R.; Gigli, G., Microfluidic motion for a direct investigation of the structural dynamics of glass-forming liquids. Anal. Chem, 2004; Vol. 77, pp 591-595.

4. Girardo, S.; Cingolani, R.; Pisignano, D., Investigating the temperature dependence of the viscosity of a non-Newtonian fluid within lithographically defined microchannels. The Journal of Chemical Physics, 2007; Vol. 127.

5. Girardo, S.; Cingolani, R.; Pisignano, D., Microfluidic Rheology of non-Newtonian liquids. Anal. Chem, 2007; Vol. 79, pp 5856-5861.

6. Han, Z.; Zheng, B., A poly(dimethylsiloxane) viscometer for microliter power law fluids. Journal of Micromethanics and Microengineering, 2009; Vol. 19, p 115005.

7. Miller, C., Predicting non-Newtonian flow behavior in ducts of unusual cross section. Industrial \& Engineering Chemistry Fundamentals, 1972; Vol. 11, pp 524-528.

8. Bahrami, M.; Yovanovich, M. M.; Culham, J. R., Pressure drop of fully-developed laminar flow in microchannels of arbitrary cross-section. Journal of Fluids Engineering, 2006; Vol. 128, pp 1036-1044.

9. Muzychka, Y. S.; Edge, J., Laminar non-Newtonian fluid flow in noncircular ducts and microchannels. Journal of Fluids Engineering, 2008; Vol. 130. 
CHAPTER 4

The Use of Phospholipids for Non-Mechanical

Flow Control and Injection in Microfluidics 


\subsection{Introduction}

Fluid control in microfluidic devices facilitates portable or remote analyses. A variety of passive and active strategies are used to accomplish fluid control. ${ }^{1}$ By far, the most prevalent method of active fluid gating is based on PDMS-elastomeric valves that deform to close fluid channels. ${ }^{2}$ These valves require a separate set of addressable channels that provide a path for pressurized gas to displace the elastomer. A different strategy for fluid control is based on thermally responsive materials, such as co-block polymers. ${ }^{3}$ In the case of co-block polymer valves, fluid flow in the channel is addressed by changing the local temperature to swell the material to close the channel. When the polymer is not swollen, the channel remains open. The use of materials that are thermally responsive is promising, especially in cases where the material can also serve as the separation additive.

Two of the most advantageous characteristics of microfluidic devices are the portability of the setup and substantially reduced sample size. Reduced sample size directly corresponds to the reduced time and cost of sample collection and processing, consequently leading to fast total analysis time. Moreover, in biomedical and chemical applications, such as blood glucose monitoring, minimal sample consumption and compact size are particularly important. For example, in the work done by Huang et al, ${ }^{4}$ continuous blood glucose monitoring and automatic injection of insulin in a precise and stable manner, is accomplished with a sample size ranging from $30-100 \mu \mathrm{L}$. This microfluidic device offers the advantages of small sample consumption, fast detection 
and low cost as well. The portability of the device holds high potential for mass production as a therapeutic product for diabetes patients.

Recently an increasing number of microfluidic devices have been developed for a large variety of applications such as cell culture, cell handling and clinical diagnostics, immunoassays and environmental analyses. ${ }^{5,6}$ The techniques and methods vary, but they all offer significantly reduced sample and power consumption as well as portability. In particular, the integration of capillary electrophoresis into microfluidic devices has remarkable promise. Capillary electrophoresis offers high efficiency and low sample consumption. When capillary electrophoresis is integrated with microfluidics, the device is especially suitable in situations where fast sample analysis is needed, and transporting the sample to lab for analysis is not practical. A recent example is the development of a microchip capillary electrophoresis instrument for in situ analysis in the search for extraterrestrial life. ${ }^{7,8}$ Although that set-up was designed for transport in a rocket, the valving system, based on pneumatic actuation, was large. Non-mechanical valves obviate this size requirement.

The temperature dependent, shear-thinning non-Newtonian nature of phospholipids was studied with bench-top instruments as described in Chapter Two. A home-built pressure system was used for its ready availability. However, the results revealed that there were flaws in the instrumental setup, especially the lack of thermal regulation at the detection window and end of capillary. In Chapter Three, the viscosity study has been transferred onto a microfluidic chip and the rheological behavior of phospholipids in the microchannel was characterized under ideal conditions. In that work, each piece of the 
experiment setup was temperature regulated. That is, the pressure reservoir and the glass chip were temperature controlled by three thermal electric modules (TEMs) and the PEEK tubing connecting the pressure reservoir to the glass chip was temperature controlled with a circulating water bath via a grooved copper block for heat transduction. While the study in Chapter Three emphasized the characterization of rheological behavior under ideal conditions, the goals of the study in Chapter Four were to rapidly build a prototype instrument to characterize the fluid performance in the microfluidic channel and to apply it for microfluidic applications such as flow steering and as well as sample introduction. Moreover, it is important to take the material one step further-that is to utilize it as the separation medium followed by sample introduction.

This goal can be considered as practical innovation. The work presented in this chapter is based on a paper published in Electrophoresis. ${ }^{9}$ To accomplish this, the constraints described in chapter Three must be simplified as much as possible, so that the device is used under real-world conditions. Chapter Four begins with a viscosity study with PEEK tubing thermally insulated but not thermally controlled to eliminate the cumbersome water bath in the system. This prototype instrument used bench-top pieces that can be down-sized. For fluid introduction, syringe pumps (980532, Harvard Apparatus, MA, USA) were used together with the 100 and $250 \mu \mathrm{L}$ syringe $(1710,1725$ Hamilton Company, Reno, NV). A photograph of the Hamilton syringe pump is as shown in Figure 4-1, the size of which is about 8 " by 6". This approach satisfies the purpose of prototyping. Ultimately this will be replaced with a fully programmable and extremely compact (smaller than 3" by 1") syringe pump (SPS01 Programmable Microfluidic 
Syringe Pump, LabSmith, CA, USA), which is commercially available and is able to deliver 5-100 $\mu \mathrm{L}$ solution into microfluidic systems. A photograph of the pump is shown in Figure 4-2A. Figure 4-2B shows the syringe pump on the breadboard with other microfluidic components along with a synchronized video microscope for microfluidics.

\subsection{Materials and methods}

Reagents for viscosity measurement in this chapter are the same as those detailed in Chapters Two and Three. For the viscosity measurement in chip, a setup similar to that in Chapter Three was used, except that the PEEK connection tubing was insulated with foam tape but was not temperature controlled. The temperature of the room was $21^{\circ} \mathrm{C}$ during the experiments. For the reader's convenience, all the reagents and materials used in the chapter are listed below.

\subsubsection{Reagents and materials.}

Maltooligosaccharide standards and 3-(N-morpholino)propanesulfonic acid (MOPS) were purchased from Sigma-Aldrich (St. Louis, MO, USA). Biotium (Hayward, CA, USA) was the source for 1-aminopyrene-3,6,8-trisulfonic acid (APTS). Fluorescein isothiocyanate (FITC) was purchased from Thermo Fisher Scientific Inc. (Rockford, IL, USA). The phospholipids DMPC and DHPC were purchased from Avanti Polar Lipids (Alabaster, AL, USA). Deionized water was generated from an Elga Purelab ultra water system (Lowell, MA, USA). The phospholipid preparations were comprised of DMPC and DHPC for which $[\mathrm{DMPC}] /[\mathrm{DHPC}]=2.5$. The preparations were dissolved in $50 \mathrm{mM}$ MOPS, buffered at $\mathrm{pH}$ of 7 to achieve an aqueous solution that consists of $20 \% \mathrm{w} / \mathrm{v}$ 
total phospholipid. The preparation was subjected to three freeze-thaw cycles. The solution was centrifuged for 10 minutes at 10,000 RPM. Linear maltooligosaccharides were labeled with APTS using a procedure reported previously. ${ }^{10}$ 


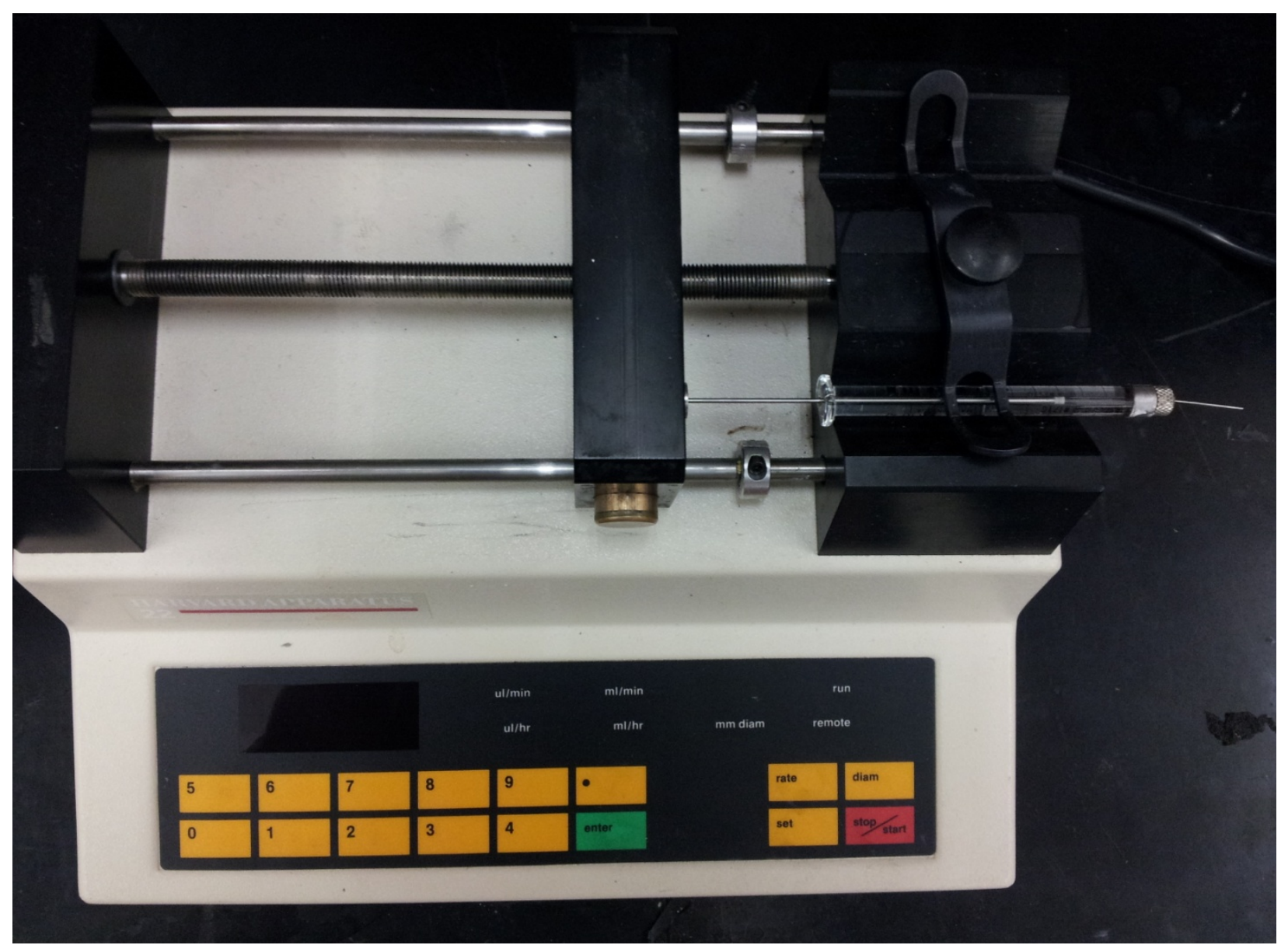

Figure 4-1. A photograph of the syringe pump (980532, Harvard Apparatus, MA, USA) used to develop the prototype instrument. This instrument is about 8" by 6" and can be reduced in size using the syringe pump as shown in Figure 4-2. 


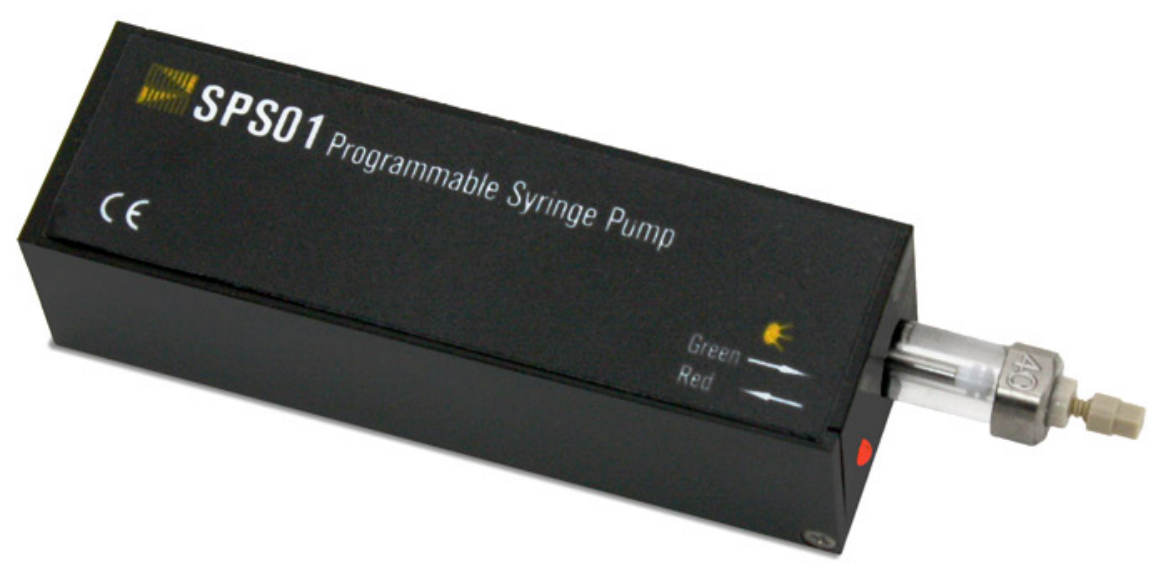

Figure 4-2A. An image of LabSmith SPS01 Programmable Syringe pump (Courtesy of LabSmith)

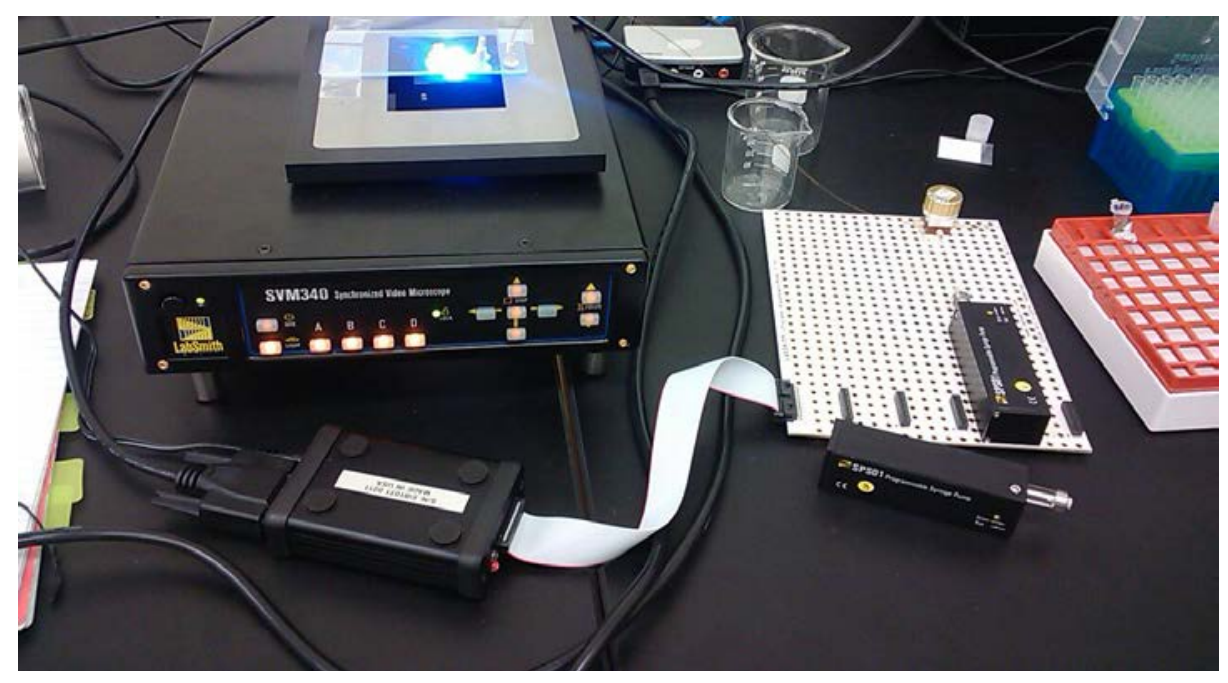

Figure 4-2B. The LabSmith syringe pump on a breadboard with other microfluidic components 
Viscosity measurements and fluid steering were performed using a microfluidic chip visualized with a Nikon Eclipse TE300 inverted microscope (Vermont Optechs, Inc., Charlotte, VT, USA) as shown in Chapter Three. The designs of the two different microfluidic chips are displayed in Figure 4-3 A and B. The microfluidic chip was fabricated using standard photolithography and wet chemical etching with a chrome photomask on borosilicate substrate $(2 \times 4$ inch Telic, Valencia,CA, USA). The crosssectional geometry was measured before chip bonding using Alpha-Step IQ surface profiler (KLA Tencor, CA, USA). NanoPorts (6-32 FB, Upchurch Scientific, WA, USA) were connected to each reservoir on the chip with epoxy. The chip was mounted on a thermally regulated stage of a Nikon Eclipse TE300 inverted microscope (Vermont Optechs, Inc., VT, USA) equipped with a Hamamatsu C10600 ORCA-R ${ }^{2}$ digital camera (Hamamatsu Photonics, K.K., Hamamatsu City, Japan). The temperature of the microfluidic chip was controlled by thermoelectric modules (TEM) manufactured by Ferrotec $(\mathrm{NH}, \mathrm{USA})$ that were each in contact with a heat sink. A high flow computer fan delivered air to the thermal fins of the heat sink. In order to flush the microfluidic chip, PEEK ${ }^{\mathrm{TM}}$ tubing (Upchurch Scientific, WA, USA) was used to connect the chip port to a stainless steel pressure reservoir or syringe pump (980532, Harvard Apparatus, MA, USA) (Figure 4-1). The chip was flushed with $3 \mathrm{~mL}$ water, $0.5 \mathrm{~mL}$ MOPS, and then filled with phospholipid.

For viscosity measurements performed in the microfluidic chip, the chip design is as shown in Figure 4-3B. Fluorescent sample was loaded into the separation channel 
Figure 4-3A. Top view of chip used for sequential and repetitive switching
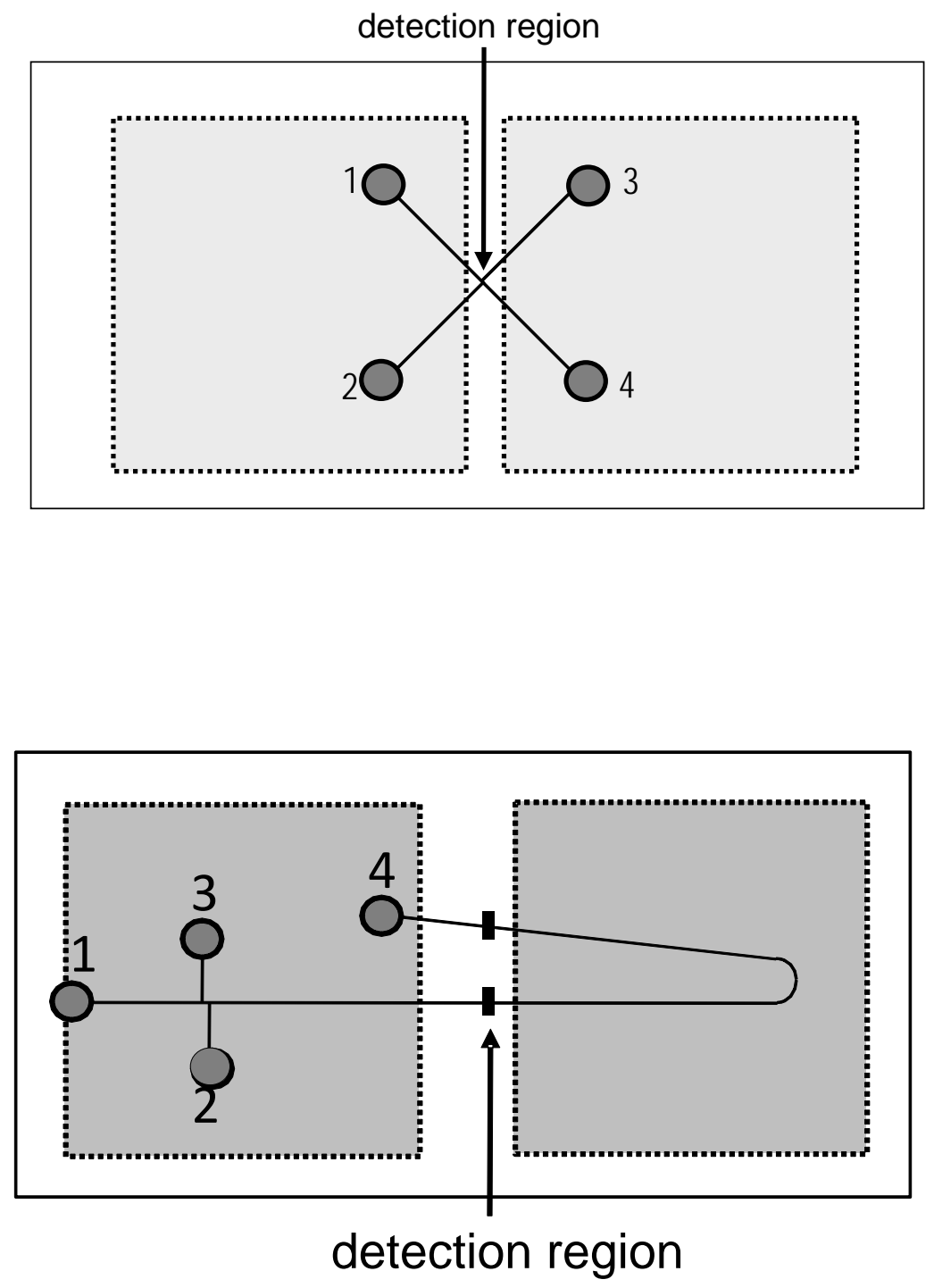

Figure 4-3B. Top view of chip used for viscosity study as well as sample introduction and separation. Fluorescent sample plug was injected through port 2 and port 3. For viscosity measurement, phospholipids were delivered to port 1 driven by pressure. For the capillary electrophoresis separation, two electrodes were inserted in port 1 and 4. 
electrokinetically, so that transport to the detection window was due solely to the pressure-driven fluid flow initiated at the beginning of the run. To accomplish this, the channel was loaded with phospholipids. Ports 1,3 , and 4 were filled with $30 \mu \mathrm{L}$ of MOPS, while port 2 was filled with $30 \mu \mathrm{L}$ of APTS dissolved in $50 \mathrm{mM}$ MOPS buffered to $\mathrm{pH}$ 7. The APTS was electrokinetically injected from port 2 to 3 for 30 seconds at -1.0 kV with a high voltage power supply (CZE1000R, Spellman High Voltage Electronics, NY, USA).The solution that filled ports 2 and 3 was removed and the ports blocked. The APTS plug was used to visualize the velocity. The APTS plug was then driven through the channel to port 4 from the pressure driven flow applied at port 1 . The APTS plug elution time was determined at a specific temperature and applied pressure at a defined position in the chip. For thermally actuated fluid steering performed in microfluidic chips, either a pressure reservoir or a syringe pump was used to introduce fluid to the ports as described in the text. Figure 4-4 is a photograph showing the temperature control with TEMs of the microfluidic channels .Fluorescence was imaged with APTS labeled oligosaccharides or APTS doped DMPC-DHPC preparations. Data were processed using HClmage version 1.1.3.1 (Hamamatsu Photonics, K.K., Hamamatsu City, Japan). To estimate figures of merit, the total amount of videocaptured grayscale in a region of the channel was exported to Microsoft Office Excel 2007 (Microsoft Corporation, Redmond, WA, USA) from HCImage. The data set was then imported into IGor-Pro version 4.0 (WaveMetrics, Inc., Lake Oswego, OR, USA ) and fit to a Gaussian in order to calculate theoretical plate count. 


\subsubsection{Beckman P/ACE capillary electrophoresis instrument}

Beckman PIACE MDQ (Beckman Coulter, Fullerton, CA,USA) was used with a $40.2 \mathrm{~cm}$ long, 25 micrometer inner diameter capillary, 360 micrometer outer diameter (Polymicro Technologies, Phoenix, AZ,USA). The distance to the detection window was $10.2 \mathrm{~cm}$.

Sample was injected electrokinetically ( $2 \mathrm{kV}, 6 \mathrm{~s})$ and separated with a field strength of $250 \mathrm{~V} / \mathrm{cm}$. Theoretical plates are calculated using 32 Karat Software version 5.0 (Beckman Coulter) using the "USP Plates" correction. 


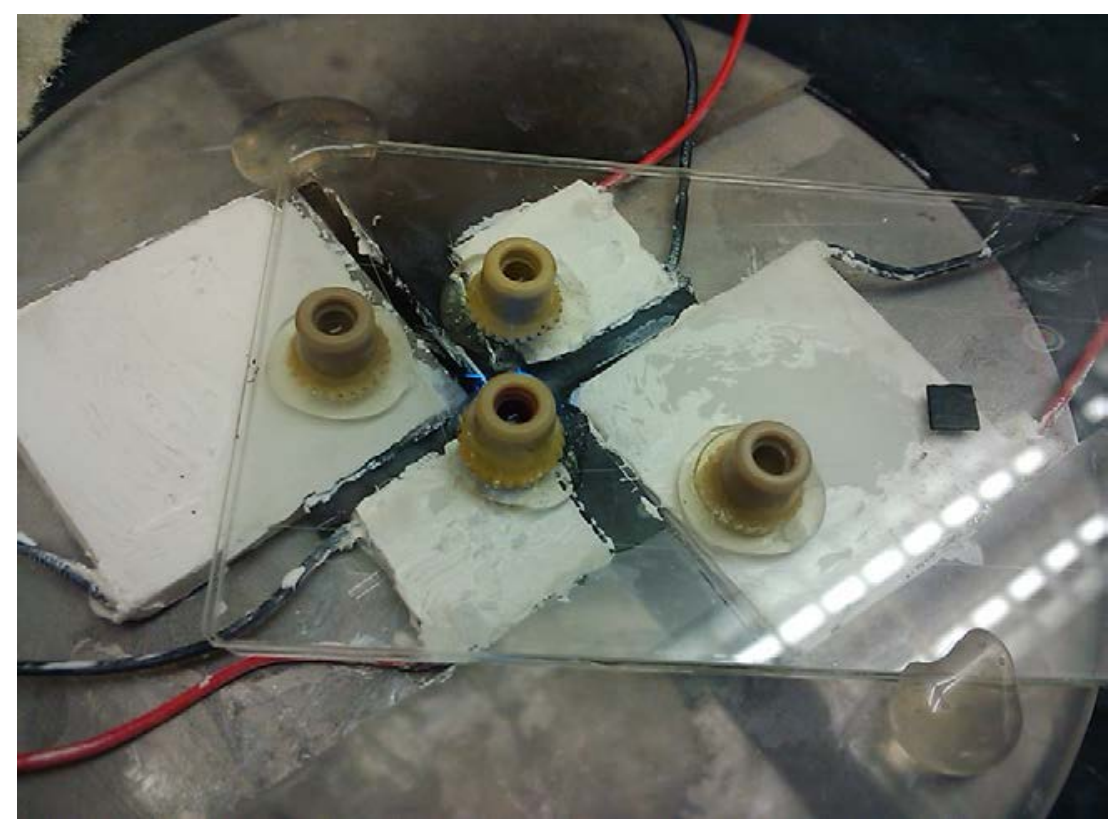

Figure 4-4. A photograph showing a microfluidic chip on the microscope stage.

A TEM was placed under each channel for temperature control. A crossshaped spacer was used to align the TEMs. There was an opening in the center of the cross-shaped spacer for detection. The Plexiglas spacer was painted black to eliminate light scattering. 


\subsection{Results and discussion}

\subsubsection{Viscosity characterization}

The viscosity characterization of $20 \%$ DMPC-DHPC in chip was performed under conditions similar to the flow steering experiments. The experiment setup was as detailed in Chapter Three except that the PEEK tubing was insulated but not temperature controlled. The room temperature was $21^{\circ} \mathrm{C}$ during the experiment. The measured apparent viscosity values at each shear rate as well as the fitting results for $\mathrm{K}$ and $\mathrm{n}$ values at $23^{\circ} \mathrm{C}, 24^{\circ} \mathrm{C}, 26^{\circ} \mathrm{C}$ and $29^{\circ} \mathrm{C}$ are summarized in Table 4-1 to show the influence of the room temperature through the PEEK tubing. As shown in Table 4-1, while the material still displays shear-thinning behavior at all temperatures (indicated by $n$ value less than 1), the material is a more shear-thinning fluid at $26^{\circ} \mathrm{C}$ and $29^{\circ} \mathrm{C}$ than at $23^{\circ} \mathrm{C}$ and $24^{\circ} \mathrm{C}$. At the lowest shear rate of $24^{\circ} \mathrm{C}$ and $26^{\circ} \mathrm{C}$, obtained with 2 psi applied pressure, the apparent viscosity at $26^{\circ} \mathrm{C}$ is approximately 40 times the viscosity at $24{ }^{\circ} \mathrm{C}$. Figure $4-5$ displays this shear-thinning viscosity, in which the viscosities are plotted against shear rate at each temperature on a log-log scale. Table 4-2 and Table 4-3 compare the results obtained when the PEEK tubing temperature is controlled and not controlled. While it is clear that the effect of the temperature control on PEEK tubing is not negligible, the data indicate that under conditions that PEEK tubing temperature is uncontrolled phospholipids can provide sufficient viscosity change to be applied to flow steering in microfluidics. 


\section{Table 4-1.}

Apparent Viscosity for 20\% Phospholipids in Microfluidic Ducts (PEEK not controlled)

$23^{\circ} \mathrm{C}, \mathrm{L}=3.2 \mathrm{~cm}$

$\mathrm{n}=0.76 \pm 0.06 \quad \mathrm{~K}=0.3 \pm 0.1 \quad \mathrm{R}^{2}=0.984$

\begin{tabular}{ll}
$\begin{array}{l}\text { Shear rate }\left(\mathbf{s}^{-1}\right) \\
\text { range }=60-3000\end{array}$ & Appt viscosity (Pa.s) \\
$3000 \pm 1000$ & $0.05 \pm 0.03$ \\
$1800 \pm 500$ & $0.05 \pm 0.03$ \\
$1300 \pm 400$ & $0.06 \pm 0.03$ \\
$800 \pm 200$ & $0.06 \pm 0.04$ \\
$600 \pm 200$ & $0.07 \pm 0.04$ \\
$340 \pm 50$ & $0.08 \pm 0.04$ \\
$140 \pm 10$ & $0.09 \pm 0.05$ \\
$60 \pm 10$ & $0.12 \pm 0.06$ \\
\multicolumn{2}{l}{} \\
\hline $\mathbf{2 6}{ }^{\circ} \mathbf{C}, \mathrm{L}=\mathbf{3 . 2} \mathbf{~ c m}$ \\
\hline $\mathrm{n}=0.54 \pm 0.04 \quad \mathrm{~K}=8 \pm 1$ & $\mathrm{R}^{2}=0.985$ \\
\hline
\end{tabular}

Shear rate $\left(\mathbf{s}^{-1}\right)$

range $=2.0-160$

$160 \pm 50$

$100 \pm 30$

$60 \pm 10$

$36 \pm 7$

$17 \pm 3$

$6.9 \pm 0.5$

$4.0 \pm 0.2$

$2.0 \pm 0.5$

\section{Appt viscosity (Pa.s)}

$0.7 \pm 0.2$

$0.9 \pm 0.3$

$1.1 \pm 0.3$

$1.5 \pm 0.4$

$2.0 \pm 0.4$

$3.1 \pm 0.6$

$4.0 \pm 0.7$

$5 \pm 1$ $24^{\circ} \mathrm{C}, \mathrm{L}=3.2 \mathrm{~cm}$

\begin{tabular}{lll}
$\mathrm{n}=0.90 \pm 0.06$ & $K=0.19 \pm 0.1$ & $\mathrm{R}^{2}=0.991$ \\
\hline
\end{tabular}
Shear rate $\left(\mathbf{s}^{-1}\right)$

range $=41-1300$

$1300 \pm 100$

$930 \pm 20$

$790 \pm 40$

$620 \pm 40$

$360 \pm 50$

$200 \pm 40$

$83 \pm 4$

$41 \pm 2$
Appt viscosity (Pa.s)

$0.09 \pm 0.05$

$0.09 \pm 0.05$

$0.09 \pm 0.05$

$0.10 \pm 0.05$

$0.10 \pm 0.05$

$0.11 \pm 0.05$

$0.12 \pm 0.05$

$0.13 \pm 0.06$

\begin{tabular}{lll}
\hline $29^{\circ} \mathrm{C}, \mathrm{L}=\mathbf{3 . 2} \mathbf{~ c m}$ \\
\hline $\mathrm{n}=0.30 \pm 0.04 \quad \mathrm{~K}=38 \pm 4$ & $\mathrm{R}^{2}=0.960$ \\
\hline
\end{tabular}

Shear rate $\left(\mathrm{s}^{-1}\right)$ range $=1.9-43$

Appt viscosity (Pa.s)

$43 \pm 9$

$2.7 \pm 0.3$

$20 \pm 3$

$4.6 \pm 0.8$

$7 \pm 2$

$10 \pm 2$

$4 \pm 1$

$14 \pm 3$

$1.9 \pm 0.4$

$24 \pm 4$

Consistency Index based on Rabinowitsch correction for circular ducts; data are fit with nonlinear regression. Triplicate measurements are performed at 8 different values of shear rate for all sets, except for $29^{\circ} \mathrm{C}$, which is based on 6 replicate measurements at 5 different shear rates. 


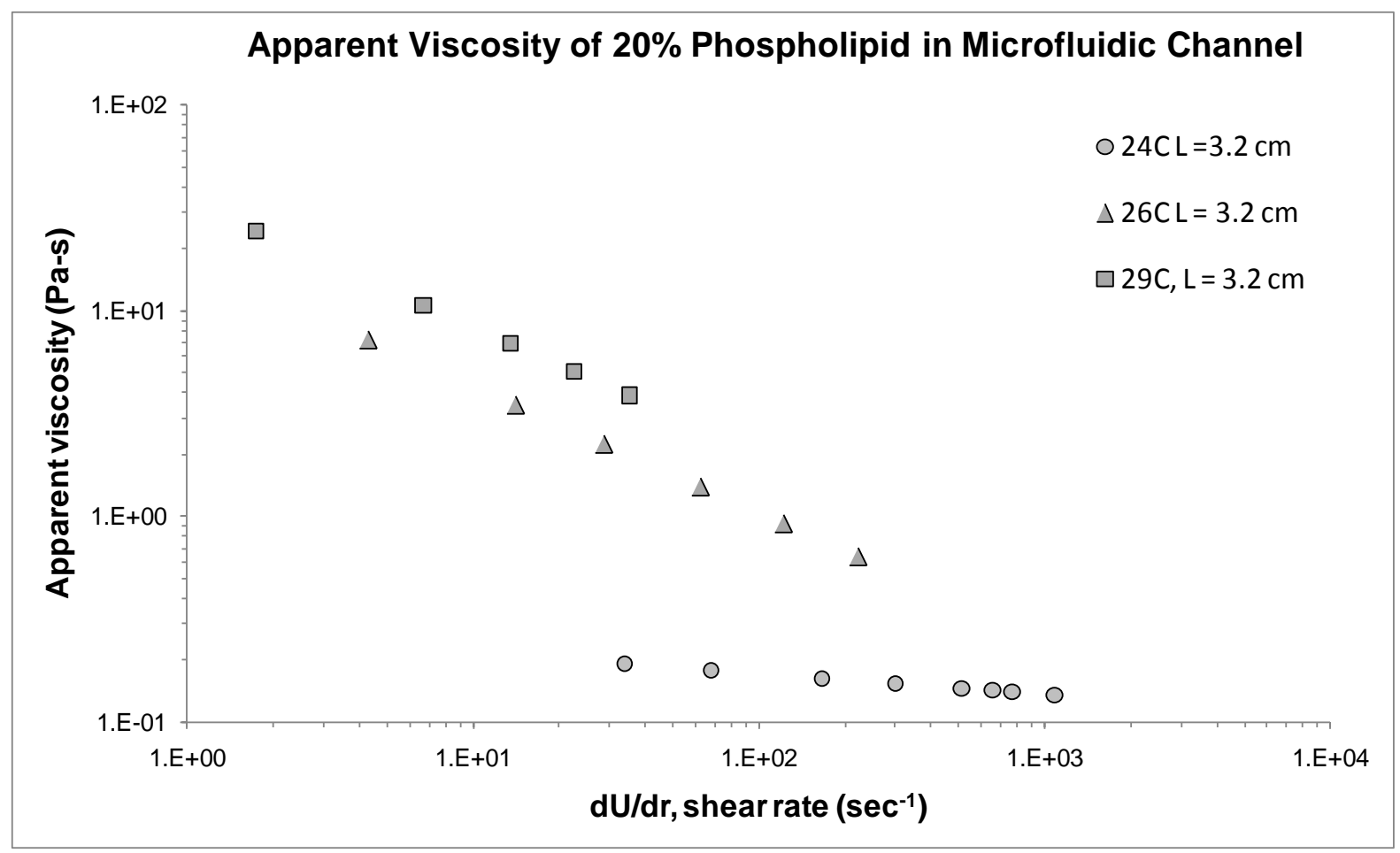

Figure 4-5 is a plot displaying the apparent viscosity of a 20\% [DMPC]/[DHPC] $=2.5$ preparation with change in shear rate. Measurements are performed in a microfluidic chip with PEEK tubing not temperature controlled as described in the text. It is shown that apparent viscosity decreases as shear rate increases to a much greater degree at $26{ }^{\circ} \mathrm{C}$ and $29^{\circ} \mathrm{C}$ than $24^{\circ} \mathrm{C}$, which indicate that the shear-thinning nature of phospholipids is more pronounced at temperatures above gel-liquid crystalline transition temperature of DMPC. It is also shown that at low shear rate, there is a significant difference in viscosity between $24^{\circ} \mathrm{C}$ and $26^{\circ} \mathrm{C}$, which is the foundation of the following applications in microfluidics. 


\begin{tabular}{|c|c|c|c|c|c|}
\hline & $n$ & K & & $n$ & K \\
\hline $\begin{array}{l}23^{\circ} \mathrm{C} \\
\text { Controlled }\end{array}$ & $0.51 \pm 0.03$ & $4.0 \pm 0.7$ & $\begin{array}{l}23^{\circ} \mathrm{C} \\
\text { Uncontrolled }\end{array}$ & $0.76 \pm 0.06$ & $0.3 \pm 0.1$ \\
\hline $\begin{array}{l}24^{\circ} \mathrm{C} \\
\text { Controlled }\end{array}$ & $0.59 \pm 0.02$ & $3.7 \pm 0.5$ & $\begin{array}{l}24{ }^{\circ} \mathrm{C} \\
\text { Uncontrolled }\end{array}$ & $0.90 \pm 0.06$ & $0.2 \pm 0.1$ \\
\hline $\begin{array}{l}26^{\circ} \mathrm{C} \\
\text { Controlled }\end{array}$ & $0.29 \pm 0.03$ & $40 \pm 6$ & $\begin{array}{l}26^{\circ} \mathrm{C} \\
\text { Uncontrolled }\end{array}$ & $0.54 \pm 0.04$ & $8 \pm 1$ \\
\hline $\begin{array}{l}29^{\circ} \mathrm{C} \\
\text { Controlled }\end{array}$ & $0.29 \pm 0.04$ & $56 \pm 8$ & $\begin{array}{l}29^{\circ} \mathrm{C} \\
\text { Uncontrolled }\end{array}$ & $0.30 \pm 0.04$ & $38 \pm 4$ \\
\hline
\end{tabular}

Table 4-2 compares the $n$ and $\mathrm{K}$ values from fitting results obtained in trapezoidal ducts with PEEK tubing temperature controlled and uncontrolled. For values recorded at 23 ${ }^{\circ} \mathrm{C}, 24^{\circ} \mathrm{C}$ and $26^{\circ} \mathrm{C}$, uncontrolled measurement gave higher $n$ values while $\mathrm{n}$ values are close at $29^{\circ} \mathrm{C}$. The difference can be attributed to the influence of room temperature which was around $21^{\circ} \mathrm{C}$. This is lower than the gel phase transition temperature. The cooler room temperature results in shorter elution time, and this effect is more pronounced at lower shear rate, which is the cause of higher $n$ value when PEEK tubing is not controlled. At $29^{\circ} \mathrm{C}$ this effect is less evident because the viscosity at $29^{\circ} \mathrm{C}$ is very high and influence is relatively small. $\mathrm{K}$ value is indicative of the apparent viscosity values which are listed in Table 4-3. 
Table 4-3. Comparison of Apparent Viscosity Obtained in Trapezoidal Ducts (q 2.5, 20\%) PEEK Tubing Temperature Controlled vs. Uncontrolled

\begin{tabular}{ll}
\hline $\begin{array}{l}23{ }^{\circ} \mathrm{C} \text { Controlled } \\
\text { Before Turn, } \mathrm{L}=3.2 \mathrm{~cm}\end{array}$ \\
\hline $\mathrm{n}=0.51 \pm 0.03, \mathrm{~K}=4.0 \pm 0.7 \mathrm{~Pa} \cdot \mathrm{s}^{\mathrm{n}}, \mathrm{R}^{2}=0.994$ \\
$\begin{array}{l}\text { Shear rate }\left(\mathrm{s}^{-1}\right) \\
\text { range }=9-2100\end{array}$ & Appt viscosity (Pa.s) \\
$2100 \pm 400$ & $0.09 \pm 0.03$ \\
$1300 \pm 200$ & $0.12 \pm 0.03$ \\
$800 \pm 20$ & $0.15 \pm 0.04$ \\
$420 \pm 70$ & $0.20 \pm 0.05$ \\
$240 \pm 20$ & $0.27 \pm 0.06$ \\
$78 \pm 6$ & $0.5 \pm 0.1$ \\
$20 \pm 6$ & $0.9 \pm 0.2$ \\
$9 \pm 4$ & $1.4 \pm 0.4$ \\
& \\
$\mathbf{2 4}{ }^{\circ} \mathrm{C} \mathrm{Controlled}$ & \\
Before Turn, $\mathrm{L}=3.2 \mathrm{~cm}$ & \\
\hline $\mathrm{n}=0.59 \pm 0.02, \mathrm{~K}=3.7 \pm 0.5 \mathrm{~Pa} \cdot \mathrm{s}^{\mathrm{n}}, \mathrm{R}^{2}=0.997$ \\
Shear rate $\left(\mathrm{s}^{-1}\right)$ & Appt viscosity (Pa.s) \\
range $=8-800$ & \\
$800 \pm 200$ & $0.24 \pm 0.06$ \\
$570 \pm 90$ & $0.27 \pm 0.06$ \\
$390 \pm 80$ & $0.32 \pm 0.07$ \\
$250 \pm 40$ & $0.38 \pm 0.08$ \\
$110 \pm 30$ & $0.5 \pm 0.1$ \\
$24 \pm 2$ & $1.0 \pm 0.2$ \\
$12 \pm 1$ & $1.3 \pm 0.2$ \\
$8 \pm 2$ & $1.6 \pm 0.3$ \\
&
\end{tabular}

\begin{tabular}{|c|c|}
\hline \multicolumn{2}{|c|}{$n=0.76 \pm 0.06, K=0.3 \pm 0.1 \mathrm{~Pa} \cdot \mathrm{s}^{\mathrm{n}}, \mathrm{R}$} \\
\hline $\begin{array}{l}\text { Shear rate }\left(s^{-1}\right) \\
\text { range }=60-3000\end{array}$ & Appt viscos \\
\hline $3000 \pm 100$ & $0.05 \pm 0.03$ \\
\hline $1800 \pm 500$ & $0.05 \pm 0.03$ \\
\hline $1300 \pm 400$ & $0.06 \pm 0.03$ \\
\hline $800 \pm 200$ & $0.06 \pm 0.04$ \\
\hline $600 \pm 200$ & $0.07 \pm 0.04$ \\
\hline $340 \pm 50$ & $0.08 \pm 0.04$ \\
\hline $140 \pm 10$ & $0.09 \pm 0.05$ \\
\hline $60 \pm 10$ & $0.12 \pm 0.06$ \\
\hline
\end{tabular}

\section{$24^{\circ} \mathrm{C}$ Uncontrolled}

$\mathrm{n}=0.90 \pm 0.06, \mathrm{~K}=0.2 \pm 0.1 \mathrm{~Pa} \cdot \mathrm{s}^{\mathrm{n}}, \mathrm{R}^{2}=0.991$

Shear rate $\left(\mathrm{s}^{-1}\right)$

range $=41-1300$

$\begin{array}{ll}1300 \pm 100 & 0.09 \pm 0.05 \\ 930 \pm 20 & 0.09 \pm 0.05 \\ 790 \pm 40 & 0.09 \pm 0.05 \\ 620 \pm 40 & 0.10 \pm 0.05 \\ 360 \pm 50 & 0.10 \pm 0.05 \\ 200 \pm 40 & 0.11 \pm 0.05 \\ 83 \pm 4 & 0.12 \pm 0.05 \\ 41 \pm 2 & 0.13 \pm 0.06\end{array}$




\section{$26^{\circ} \mathrm{C}$ Controlled}

Before Turn, $L=3.2 \mathrm{~cm}$

$\begin{array}{ll}\mathrm{n}=0.29 \pm 0.03, K=40 \pm 6 \mathrm{~Pa} \cdot \mathrm{s}^{\mathrm{n}}, \mathrm{R}^{2}=0.968 \\ \begin{array}{l}\text { Shear rate }\left(\mathrm{s}^{-1}\right) \\ \text { range }=0.52-220\end{array} & \text { Appt viscosity (Pa.s) } \\ 220 \pm 20 & 0.9 \pm 0.2 \\ 110 \pm 20 & 1.4 \pm 0.3 \\ 37 \pm 5 & 3.0 \pm 0.6 \\ 11 \pm 1 & 7 \pm 1 \\ 3.6 \pm 0.3 & 16 \pm 2 \\ 1.4 \pm 0.1 & 31 \pm 5 \\ 0.52 \pm 0.04 & 60 \pm 10\end{array}$

\section{$29{ }^{\circ} \mathrm{C}$ Controlled}

Before Turn, $L=3.2 \mathrm{~cm}$

\begin{tabular}{|c|c|}
\hline \multicolumn{2}{|c|}{$\mathrm{n}=0.29 \pm 0.04, K=56 \pm 8 \mathrm{~Pa} \cdot \mathrm{s}^{\mathrm{n}}, \mathrm{R}^{2}=0.936$} \\
\hline $\begin{array}{l}\text { Shear rate }\left(\mathbf{s}^{-1}\right) \\
\text { range }=0.98-75\end{array}$ & Appt viscosity (Pa.s) \\
\hline $75 \pm 8$ & $2.6 \pm 0.6$ \\
\hline $30 \pm 4$ & $5 \pm 1$ \\
\hline $11 \pm 2$ & $10 \pm 2$ \\
\hline $3.2 \pm 0.3$ & $25 \pm 4$ \\
\hline $1.7 \pm 0.1$ & $39 \pm 6$ \\
\hline $0.98 \pm 0.08$ & $57 \pm 9$ \\
\hline
\end{tabular}

$26^{\circ} \mathrm{C}$ Uncontrolled

$\mathrm{n}=0.54 \pm 0.04, \mathrm{~K}=8 \pm 1 \mathrm{~Pa} \cdot \mathrm{s}^{\mathrm{n}}, \mathrm{R}^{2}=0.985$

Shear rate $\left(\mathbf{s}^{-1}\right)$

Appt viscosity (Pa.s)

range $=2.0-160$

$\begin{array}{lc}160 \pm 50 & 0.7 \pm 0.2 \\ 100 \pm 30 & 0.9 \pm 0.3 \\ 60 \pm 10 & 1.1 \pm 0.3 \\ 36 \pm 7 & 1.5 \pm 0.4 \\ 17 \pm 3 & 2.0 \pm 0.4 \\ 6.9 \pm 0.5 & 3.1 \pm 0.6 \\ 4.0 \pm 0.2 & 4.0 \pm 0.7 \\ 2.0 \pm 0.5 & 5 \pm 1\end{array}$

$29^{\circ} \mathrm{C}$ Uncontrolled

$n=0.30 \pm 0.04, K=38 \pm 4 \mathrm{~Pa} \cdot \mathrm{s}^{\mathrm{n}}, \mathrm{R}^{2}=0.960$

Shear rate $\left(\mathrm{s}^{-1}\right)$

range $=1.9-43$

$\begin{array}{ll}43 \pm 9 & 2.7 \pm 0.3 \\ 20 \pm 3 & 4.6 \pm 0.8 \\ 7 \pm 2 & 10 \pm 2 \\ 4 \pm 1 & 14 \pm 3 \\ 1.9 \pm 0.4 & 24 \pm 4\end{array}$

Consistency Index based on Rabinowitsch correction for circular ducts, data is fit with nonlinear regression 


\subsubsection{Flow steering in chip}

As the prior viscosity study revealed, the $q 2.5,20 \%$ DMPC-DHPC preparation exhibits a substantial viscosity change when the temperature changes. This feature is utilized to harness the change in flow resistance in response to the viscosity change of the lipid preparation inside the channel to steer the flow. That is, the flow passage is open when the viscosity is low at a temperature below the gel transition temperature. The passage is switched closed via greatly increased flow resistance as temperature is increased above the gel phase transition temperature.

The previously reported repetitive switching experiments were replicated using TEMs to regulate temperature. The temperature range used to direct flow was based on fluid characterization performed in the microfluidic chip. Based on that study, the viscosity required to reduce flow enough to close a channel is a function of the shear stress. At lower fluid flow rates, the flow can be stopped using lower viscosities. The temperature range required to switch to a viscosity sufficient to stop low flow rates is smaller than previously anticipated. Using a similar chip design shown in Figure 4-3A, repetitive fluid steering (Figure 4-6) was achieved using a temperature of $24^{\circ} \mathrm{C}$ to maintain open channels and $26^{\circ} \mathrm{C}$ to close channels. Controlling temperature using the thermoelectric modules decreased the response time to $2.0 \pm 0.3 \mathrm{~s}$ for valve opening, and to $8.0 \pm 0.5$ $s$ for valve closing $(n=10)$. 

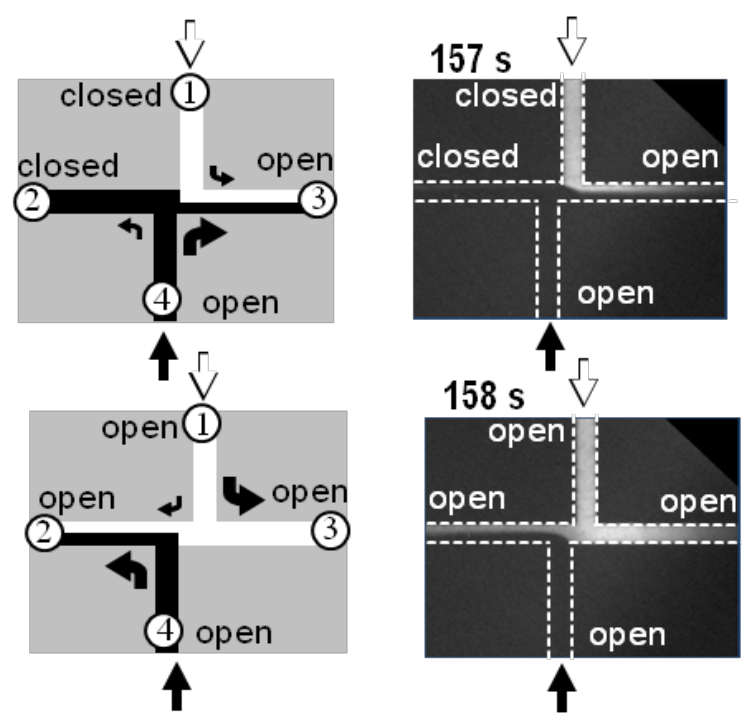

Figure 4-6 shows the steps and captured images for repetitive flow switching. Fluorescently doped lipid is delivered to port $1(1.0 \mu \mathrm{L} / \mathrm{min})$ while unlabeled phospholipid is delivered to port $4(0.8 \mu \mathrm{L} / \mathrm{min})$. Channels 1 and 2 are held closed at $26^{\circ} \mathrm{C}$ (157 seconds), or all of the channels are held open at $24^{\circ} \mathrm{C}$ (158 seconds). The channel walls are outlined with a hashed white line in the optical images for clarity. 
Sequential flow switching was also performed using a chip with a design similar to that used for repetitive flow switching. Figure 4-7 shows the steps and images for sequential switching. While channels 1 and 3 are temperature regulated by one TEM, channels 2 and 4 are each individually controlled by a smaller sized TEM. At the beginning of the experiment, the four channels were filled with clear DMPC-DHPC preparations of $[\mathrm{DMPC}] /[\mathrm{DHPC}]=2.5,20 \%$ hydration while all the channels were open. Then with channels 1 and 2 at a temperature such that flow was stopped, fluorescently doped DMPC-DHPC was delivered via port 4 and was directed to channel 3 only. Figure 4-7A shows the steps for repetitive flow switching. Fluorescently doped lipid was delivered to port $1(1.0 \mu \mathrm{L} / \mathrm{min})$ while unlabeled phospholipid is delivered to port $4(0.8 \mu \mathrm{L} / \mathrm{min})$. Channels 1 and 2 were held closed at $26^{\circ} \mathrm{C}(157 \mathrm{~s})$, or all of the channels are held open at $24{ }^{\circ} \mathrm{C}(158 \mathrm{~s})$. The channel walls were outlined with a hashed white line in the optical images for clarity. The images shown in Figure 4-7B at 30 seconds, 48 seconds, and 56 seconds capture the sequential selection of fluid flow to a different channel. 

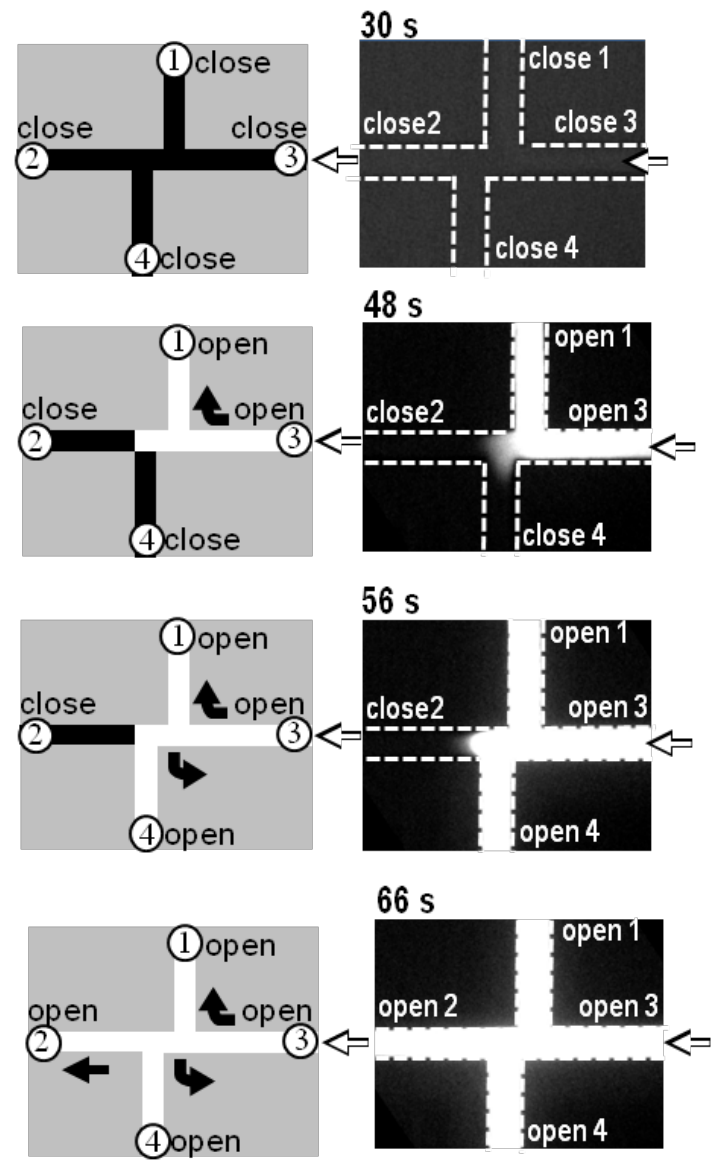

Figure 4-7. Steps and captured images for sequential flow switching.

Initially all channels are maintained at $26^{\circ} \mathrm{C}$. Fluorescently doped phospholipid is delivered through port 3 at $0.6 \mu \mathrm{L} / \mathrm{min}$. Channels are switched from $26^{\circ} \mathrm{C}$ to $19^{\circ} \mathrm{C}$ in the following order: channels 3 and 1 , then channel 4 , and finally channel 2 . The images shown at 30 seconds, 48 seconds, and 56 seconds capture the sequential selection of fluid flow to a different channel. 


\subsubsection{Thermally facilitated hydrodynamic injections}

To expand upon the use of repetitive switching, the material was also utilized for thermally facilitated hydrodynamic injections in microfluidic channels. The principle drawback to hydrodynamic injection in chip is that the fluid resistance, which is a function of channel diameter and length, is too low for pressures that can be practically applied to deliver typical aqueous solutions into the chip. The switchable viscosity as shown in Figure 4-5 solves this dilemma. In this example, phospholipid is introduced at channel 4 at $0.5 \mu \mathrm{L} / \mathrm{min}$. Channels 1,2 , and 4 are maintained at temperatures ranging from $20-21^{\circ} \mathrm{C}$, but channel 3 is maintained at $25^{\circ} \mathrm{C}$. Sample solution is introduced at channel 1, when a syringe pump set to deliver fluorescently doped phospholipid at 2 $\mu \mathrm{L} / \mathrm{min}$ is turned on for 10 seconds. The temperature difference and balance of fluid flow rate enable the introduction of a small fluorescent plug into channel 4 (see the frames captured at 129 and 138 seconds). The process of thermally assisted injection was repeated with 10 manual injections of fluorescently labeled sample plug as shown in the grayscale plot in Figure 4-8. If hydrodynamic injections with thermally assisted fluid steering are successfully integrated with chip-based electrophoresis, the method could expand the applicability of microfluidic separations for rapid introduction of different analytical samples and higher throughput sample analyses in microfluidics. 


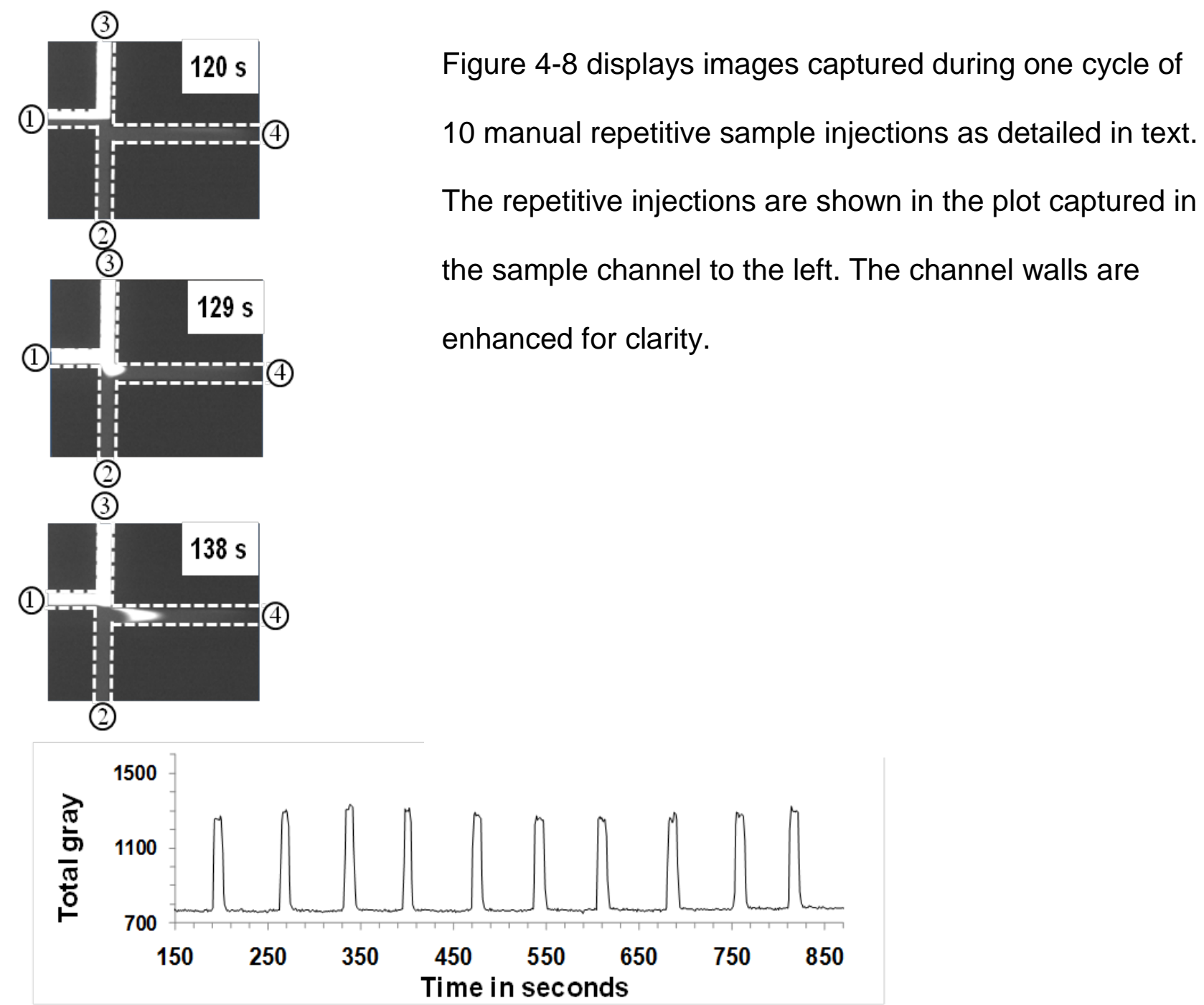


4.3.4 Hydrodynamic sample introduction and capillary electrophoresis separation

The thermally responsive apparent viscosity of phospholipids was utilized to enable hydrodynamic injection of analyte in the chips followed by separation. The process involves introducing sample at low temperature through port 2 and port 3, while maintaining ports 1 and 4 at higher temperature to close and port 4 at low temperature such that it is open. The sample is directed from port 2 to 3 , across the double T. Once the injection region of the double T is filled, all ports are closed and the injection plug is driven to the sample channel electrokinetically. Figure 4-9 demonstrates sample introduction with thermal control. For this experiment maltooligosaccharides labeled with APTS is introduced at port 3 at 2 psi. At 28.6 seconds channels 2 and 3 are maintained at $24{ }^{\circ} \mathrm{C}$ while channels 1 and 4 are maintained at $26^{\circ} \mathrm{C}$. At 33.4 seconds, the temperature at channels 2 and 3 is switched to $26^{\circ} \mathrm{C}$ to stop flow through these channels. A voltage of $-2.5 \mathrm{kV}$ is applied at channel 1 (across channel 1 and 4) at 38.2 seconds and sample is driven down the separation channel. A negative control of the experiment (see Figure 4-11) was accomplished by maintaining all channels at $24^{\circ} \mathrm{C}$. Without thermal control, all of the microfluidic channels were filled and injection into the separation channel was not feasible.

Once it was established that thermally actuated injection was possible, the detection region of the microfluidic chip was changed so that the separation can be observed. Sample injection was repeated using a mixture of three linear maltooligosaccharides (M3, M5 and M7) labeled with APTS, but with the microfluidic chip positioned to observe analyte separation. 

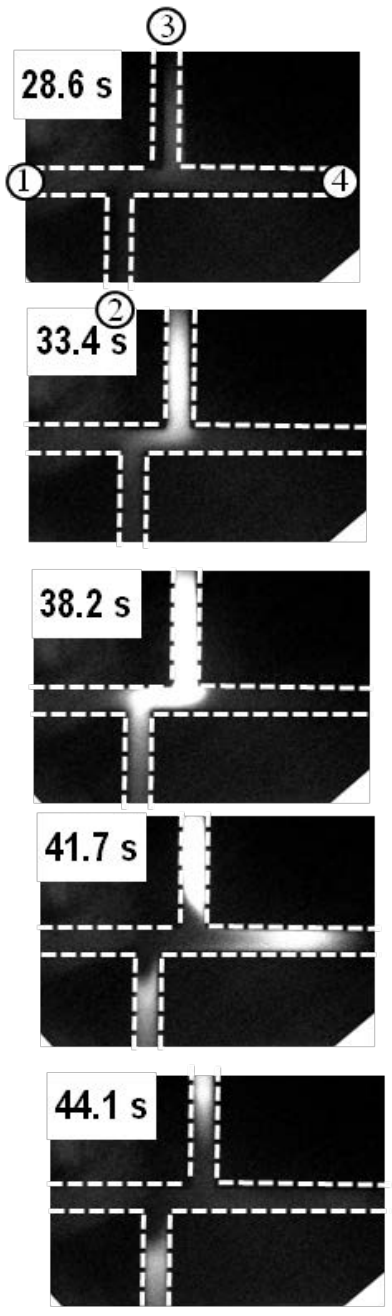

Figure 4-9 demonstrates sample introduction with thermal control. The channel walls are enhanced for clarity. Sample introduction is accomplished in the images above by maintaining channels 2 and 3 at $24{ }^{\circ} \mathrm{C}$ and channels 1 and 4 at $26^{\circ} \mathrm{C}$. APTS labeled with oligosaccharides introduced at port 3 are directed across the separation channel to port 2 (see the frames at 33.4 and 38.2 seconds). The temperature at all channels is switched to $26^{\circ} \mathrm{C}$ to stop flow so that a voltage of $-2.5 \mathrm{kV}$ applied at channel 1 will drive the injection plug down the separation channel (see the frame at 41.7 seconds). 


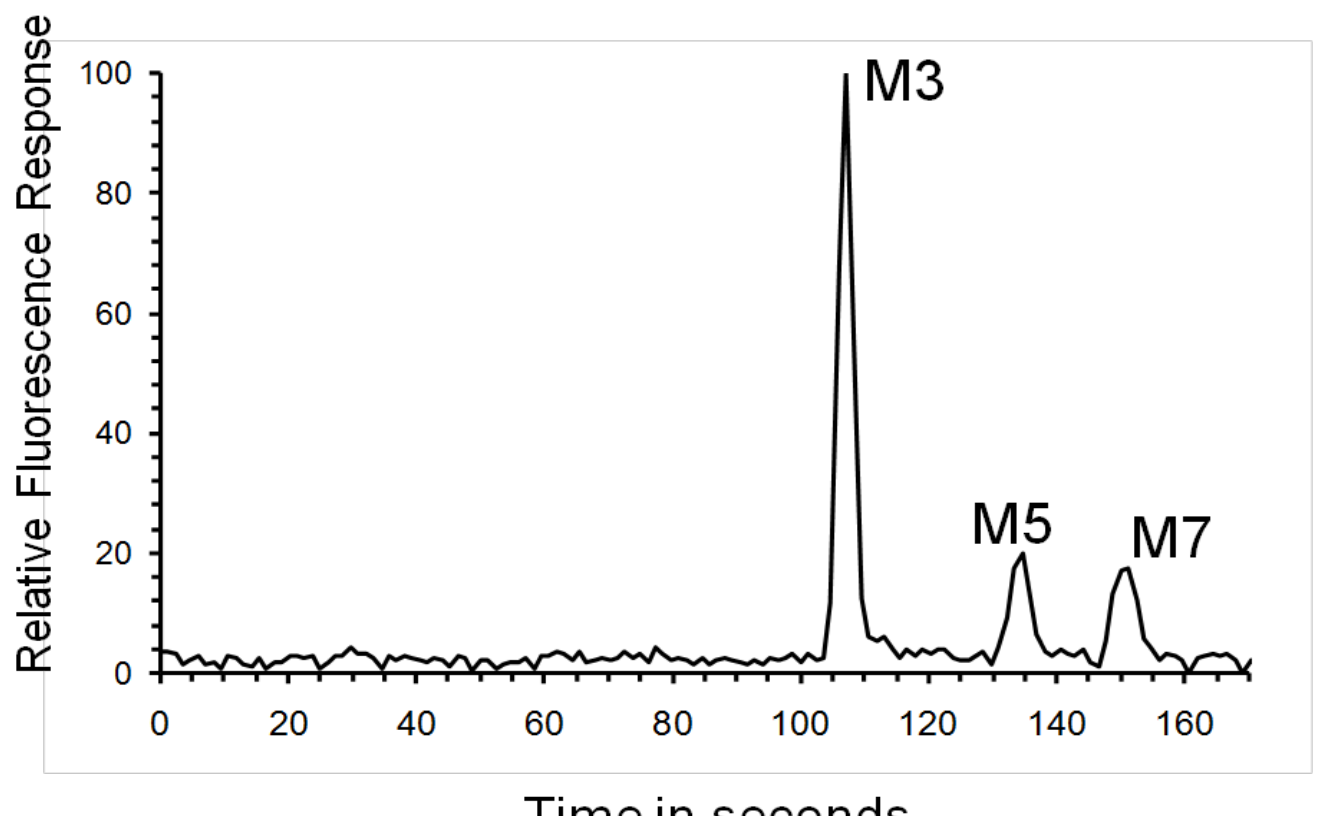

Time in seconds

Figure 4-10 shows the capillary electrophoresis separation obtained with the method of injection shown in Figure 4-9. The background electrolyte is comprised of $20 \%$ phospholipid with [DMPC]/[DHPC] $=2.5$ reconstituted in $50 \mathrm{mM}$ MOPS buffered at $\mathrm{pH} 7$. The anodic (port 1) and cathodic (port 4) reservoirs are filled with $50 \mathrm{mM}$ MOPS buffered at $\mathrm{pH} 7$ (effective length $=3.8 \mathrm{~cm}, \mathrm{E}=212 \mathrm{~V} / \mathrm{cm}$ ). 

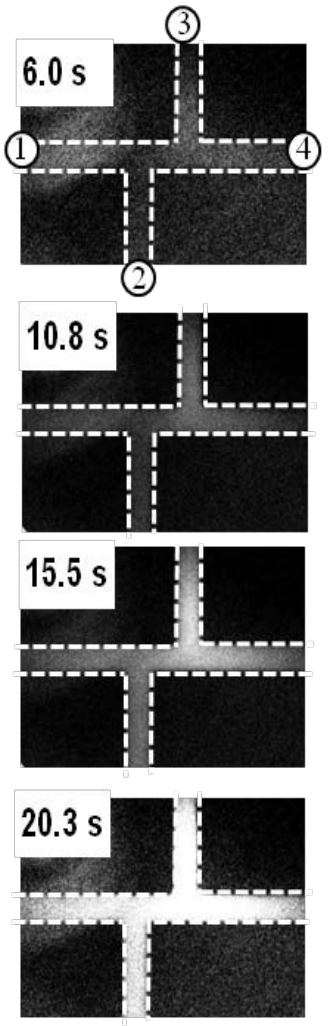

\section{$25.1 \mathrm{~s}$}

Figure $4-11$ is used as a negative control to demonstrate the difficulty of hydrodynamic injection in microfluidics. For this experiment all channels are maintained at $24^{\circ} \mathrm{C}$ during introduction of maltotriose labeled with APTS at port 3 at 2 psi. Unlike the data shown in Figure 4-9, fluorescent sample is nonselectively sent into all channels of the microfluidic chip. 
The separation is obtained with an effective length of $3.8 \mathrm{~cm}$, a field strength of 212 $\mathrm{V} / \mathrm{cm}$, and a background electrolyte comprised of $20 \%$ phospholipid with $[\mathrm{DMPC}] /[\mathrm{DHPC}]=2.5$ reconstituted in $50 \mathrm{mM}$ MOPS buffered at $\mathrm{pH}$ 7. The separation of APTS labeled maltotriose, maltopentaose, and maltoheptaose is shown in Figure 410.

Resolution (Rs) of chemical separation, calculated as the difference of retention time of two analytes divided by the average peak width, is used to evaluate how well the peaks are separated. Theoretical plate count, $\mathrm{N}$, is used to evaluate the efficiency of the separation. $\mathrm{N}$ is calculated as sixteen times retention time over peak width squared. Peaks are separated with a resolution 2.15 between M5 and M7, 3.54 between M3 and M7 and efficiency of $\mathrm{N}=3600,4160$ and 7170 for M3, M5 and M7 respectively. Three runs under the same conditions have been performed. The relative standard deviation in time for M3, M5 and M7 are within 5\%.

To investigate the thermal effect on separation, oligosaccharide separations were also carried out with the $20 \%$ phospholipid additive at different temperatures on a commercial Beckman PIACE MDQ capillary electrophoresis instrument. As shown in Table 4-4, as temperature increases from $23^{\circ} \mathrm{C}$ to $30^{\circ} \mathrm{C}$, the retention time generally decreases. This corresponds with an increase of electrophoretic mobility. However, no significant difference in resolution or theoretical plates was observed. For this reason, the on-chip capillary electrophoresis separation was carried out at $26^{\circ} \mathrm{C}$, under which temperature the injection was done. Comparing the plates/meter at $26^{\circ} \mathrm{C}$, the separation performed in capillary yield efficiencies of 550,000 for M3, 590,000 for M5 
and 590,000 for M5. On-chip separation distance of $3.8 \mathrm{~cm}$ has achieved 97,000 plates/m, 109,000 plates/m and 189,000 plates/m for M3, M5 and M7, respectively. Improvement of separation can be achieved with longer separation length and by using pinched injection to reduce the dispersion of the injection sample plug. 


\section{Table 4-4.}

Separation Figures of Merit for 20\% Phospholipid with P/ACE MDCQ

\begin{tabular}{|c|c|c|c|c|c|c|}
\hline Temperature & Time (s) & Resolution & Plates $\left(\times 10^{3}\right)$ & Plates $/ \mathrm{m}\left(\times 10^{3}\right)$ & Area $\left(\times 10^{3}\right)$ & $\mu_{\mathrm{eph}} 10^{-4} \mathrm{~cm}^{2} / \mathrm{Vs}$ \\
\hline \multicolumn{7}{|c|}{ Maltotriose $(\mathrm{M} 3)^{1}$} \\
\hline $23^{\circ} \mathrm{C}$ & $4.92 \pm 0.01$ & $7 \pm 2$ & $61 \pm 2$ & 610 & $7 \pm 1$ & 1.4 \\
\hline $24^{\circ} \mathrm{C}$ & $4.62 \pm 0.02$ & $7 \pm 1$ & $66 \pm 5$ & 660 & $5 \pm 1$ & 1.4 \\
\hline $25^{\circ} \mathrm{C}$ & $4.44 \pm 0.01$ & $7 \pm 1$ & $58 \pm 3$ & 580 & $6 \pm 0.2$ & 1.5 \\
\hline $26^{\circ} \mathrm{C}$ & $4.37 \pm 0.01$ & $6 \pm 1$ & $55 \pm 4$ & 550 & $4 \pm 0.6$ & 1.5 \\
\hline $27^{\circ} \mathrm{C}$ & $4.32 \pm 0.02$ & $7 \pm 2$ & $62 \pm 2$ & 620 & $4 \pm 0.9$ & 1.5 \\
\hline $28^{\circ} \mathrm{C}$ & $4.29 \pm 0.01$ & $6 \pm 2$ & $64 \pm 4$ & 640 & $4 \pm 0.3$ & 1.6 \\
\hline $29^{\circ} \mathrm{C}$ & $4.24 \pm 0.03$ & $7 \pm 2$ & $62 \pm 8$ & 620 & $3 \pm 0.5$ & 1.6 \\
\hline $30^{\circ} \mathrm{C}$ & $4.26 \pm 0.03$ & $8 \pm 4$ & $73 \pm 1$ & 730 & $2 \pm 0.3$ & 1.6 \\
\hline \multicolumn{7}{|c|}{ Maltopentaose(M5) ${ }^{2}$} \\
\hline $23^{\circ} \mathrm{C}$ & $5.85 \pm 0.01$ & $3 \pm 1$ & $63 \pm 4$ & 630 & $10 \pm 3$ & 1.1 \\
\hline $24^{\circ} \mathrm{C}$ & $5.45 \pm 0.02$ & $2.4 \pm 0.1$ & $61 \pm 9$ & 610 & $10 \pm 2$ & 1.2 \\
\hline $25^{\circ} \mathrm{C}$ & $5.22 \pm 0.01$ & $2.5 \pm 0.2$ & $60 \pm 2$ & 600 & $10 \pm 0.7$ & 1.3 \\
\hline $26^{\circ} \mathrm{C}$ & $5.13 \pm 0.02$ & $3 \pm 1$ & $59 \pm 7$ & 590 & $6 \pm 0.3$ & 1.3 \\
\hline $27^{\circ} \mathrm{C}$ & $5.07 \pm 0.03$ & $3.5 \pm 0.5$ & $59 \pm 3$ & 590 & $8 \pm 1$ & 1.3 \\
\hline $28^{\circ} \mathrm{C}$ & $5.04 \pm 0.01$ & $3 \pm 1$ & $62 \pm 1$ & 620 & $6 \pm 1$ & 1.3 \\
\hline $29^{\circ} \mathrm{C}$ & $4.98 \pm 0.03$ & $3 \pm 1$ & $73 \pm 9$ & 730 & $5 \pm 0.1$ & 1.3 \\
\hline $30^{\circ} \mathrm{C}$ & $5.01 \pm 0.03$ & $4 \pm 1$ & $64 \pm 7$ & 640 & $4 \pm 0.2$ & 1.3 \\
\hline \multicolumn{7}{|c|}{ Maltoheptaose(M7) } \\
\hline $23^{\circ} \mathrm{C}$ & $6.67 \pm 0.01$ & & $61 \pm 2$ & 610 & $2000 \pm 400$ & 1.0 \\
\hline $24^{\circ} \mathrm{C}$ & $6.16 \pm 0.03$ & & $62 \pm 2$ & 620 & $1000 \pm 300$ & 1.1 \\
\hline $25^{\circ} \mathrm{C}$ & $5.88 \pm 0.02$ & & $62 \pm 8$ & 620 & $2000 \pm 100$ & 1.1 \\
\hline $26^{\circ} \mathrm{C}$ & $5.77 \pm 0.02$ & & $59 \pm 1$ & 590 & $1000 \pm 100$ & 1.2 \\
\hline $27^{\circ} \mathrm{C}$ & $5.70 \pm 0.03$ & & $62 \pm 1$ & 620 & $1000 \pm 100$ & 1.2 \\
\hline $28^{\circ} \mathrm{C}$ & $5.66 \pm 0.01$ & & $64 \pm 5$ & 640 & $900 \pm 30$ & 1.2 \\
\hline $29^{\circ} \mathrm{C}$ & $5.61 \pm 0.03$ & & $63 \pm 1$ & 630 & $700 \pm 20$ & 1.2 \\
\hline $30^{\circ} \mathrm{C}$ & $5.65 \pm 0.04$ & & $61 \pm 5$ & 610 & $600 \pm 8$ & 1.2 \\
\hline
\end{tabular}

${ }^{1}$ Resolution is calculated as the difference in migration time for maltopentaose and maltotriose divided by the average width of the base of these two oligosachharides

${ }^{2}$ Resolution is calculated as the difference in migration time for maltoheptaose and maltopentaose divided by the average width of the base of these two oligosachharides 


\subsection{Conclusions and future directions}

The non-Newtonian nature of phospholipid preparations has been utilized for microfluidic separations of fluorescently labeled oligosaccharides. The material, which is a shear-thinning power-law fluid, serves as both a thermally actuated valve and an additive to enhance biomolecule separations. The findings support the use of multifunctional materials in microfluidics with potential to expand and enhance capillary electrophoresis separations. Ongoing work includes the introduction of multi-step analyte processing, multiple injections, and multiplexed sample introduction in microfluidic chips. 


\section{References}

1. Easley C, Landers JP. Practical Fluid Control Strategies for Microfluidic Devices. In: Landers JP, editor. Handbook of Capillary and Microchip Electrophoresis and Associated Microtechniques, Third Edition: CRC Press; 2007. p. 1153-68.

2. Unger MA, Chou H-P, Thorsen T, Scherer A, Quake SR. Monolithic microfabricated valves and pumps by multilayer soft lithography. Science. 2000;288:113-6.

3. Yu C, Mutlu S, Selvaganapathy P, Mastrangelo $\mathrm{CH}$, Svec F, Fréchet JMJ. Flow Control Valves for Analytical Microfluidic Chips without Mechanical Parts Based on Thermally Responsive Monolithic Polymers. Anal Chem. 2003;75(8):1958-61.

4. Huang C-J, Chen Y-H, Wang C-H, Chou T-C, Lee G-B. Integrated microfluidic systems for automatic glucose sensing and insulin injection. Sens Actuators, B, Chem. 2007;122(2):461-8.

5. Vilkner T, Janasek D, Manz A. Micro Total Analysis Systems. Recent Developments. Anal Chem. 2004;76(12):3373-86.

6. Dittrich PS, Tachikawa K, Manz A. Micro Total Analysis Systems. Latest Advancements and Trends. Anal Chem. 2006;78(12):3887-908.

7. Mora MF, Greer F, Stockton AM, Bryant S, Willis PA. Toward Total Automation of Microfluidics for Extraterrestial In Situ Analysis. Anal Chem. 2011;83(22):8636-41.

8. Benhabib M, ChiesI TN, Stockton AM, Scherer JR, Mathies RA. Multichannel Capillary Electrophoresis Microdevi` and Instrumentation for in Situ Planetary Analysis of Organic Molecules and Biomarkers. Anal Chem. 2010;82(6):2372-9.

9. Wu X, Langan T, Durney BC, Holland LA. Thermally Responsive Phospholipid Preparations for Fluid Steering and Separation in Microfluidics. Electrophoresis. 2012, 33, 2674-2681.

10. Langan TJ, Holland LA. Capillary electrophoresis coupled to electrospray mass spectrometry through a coaxial sheath flow interface and semi-permanent phospholipid coating for the determination of oligosaccharides labeled with 1aminopyrene-3,6,8-trisulfonic acid. Electrophoresis. 2012;33(4):607-13. 


\section{Xingwei Wu}

\section{Department of Chemistry}

West Virginia University

Morgantown, West Virginia 26506

xwu2@mix.wvu.edu

\section{EDUCATION}

Ph.D. in Chemistry West Virginia University, GPA: 3.9/4.0 May, 2013

Advisor: Dr. Lisa Holland

Dissertation Title: Characterization and Application of Self-Assembled Nano-materials for Non-

Mechanical Fluid Steering and Separation in Microfluidics

Area of Interests: Analytical Chemistry, Separation Chemistry, Microfluidics, lab-on-Chip, Nanotechnology

Coursework: Molecular Spectroscopy/Structure, Chemical Crystallography, Electrochemistry/Instrumentation, High Throughput Protein Analysis, Mass Spec Principles \& Practice, Chemical Separations, General Biochemistry, Bioanalytical Chemistry, SEM In Nanoscience

B.S. in Chemistry Sun Yat-Sen University, Guangzhou, China, GPA: 3.4/4.0 2005

\section{RESEARCH EXPERIENCE}

- Thermally Responsive Phospholipid Preparations for Fluid Steering and Separation in Microfluidics 2010- 2012

Xingwei Wu, Ted J. Langan, Brandon C. Durney and Lisa A. Holland, Thermally responsive phospholipid preparations for fluid steering and separation in microfluidics, Electrophoresis 2012, 33, 2674-2681

- Rheological characterization of non-Newtonian fluid phospholipids in circular and non-circular ducts (manuscript in preparation) 2009-2010

- Dielectrophoresis of Polystyrene Particles with Quadruple Electrodes 2007-2008

- Capillary Electrophoresis Distance Learning Project 2006

\section{POSTER AND PODIUM PRESENTATIONS(AS PRESENTER)}

- Rheological Characterization and Application of Phospholipid Preparations for Non-Mechanical Flow Control in Microfluidic Systems (Podium) 
Xingwei Wu, Lisa Holland 63th Pittsburgh Conference on Analytical Chemistry and Applied Spectroscopy, Orlando, FL March 2012

- Fluid Property Characterization of Phospholipids and Its Application in Non-mechanical Valving in Microfluidic Systems (Poster)

Xingwei Wu, Lisa Holland 62th Pittsburgh Conference on Analytical Chemistry and Applied Spectroscopy, Atlanta, GA March 2011

- Viscosity Study of non-Newtonian Fluid Phospholipids and its Application in non-mechanical valving in Microfluidic Systems (Podium)

Xingwei Wu, Lisa Holland 61th Pittsburgh Conference on Analytical Chemistry and Applied Spectroscopy, Orlando, FL March 2010

- On-chip non-Newtonian Fluid Phospholipids Viscosity Study and its Application in Non-mechanical Valving (Poster)

Xingwei Wu, Lisa A Holland 60th Pittsburgh Conference on Analytical Chemistry and Applied Spectroscopy, Chicago, IL March 2009

- Dielectrophoresis of Polystyrene Particles with Quadruple Electrodes Xingwei Wu, Yongkuk Lee, Lisa A Holland, R. Lloyd Carroll, Parviz Famouri 59th Pittsburgh Conference on Analytical Chemistry and Applied Spectroscopy, New Orelans, LA March 2008 